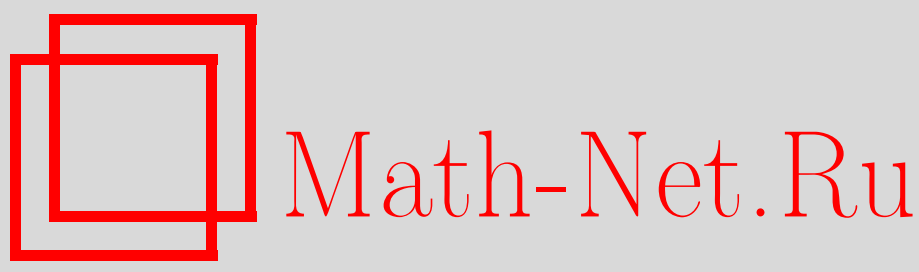

В. К. Захаров, А. В. Михалёв, Проблема общего радоновского представления для произвольного хаусдорфова пространства, Изв. РАН. Сер. матем., 1999, том 63, выпуск 5, 37-82

DOI: https://doi.org/10.4213/im265

Использование Общероссийского математического портала Math-Net.Ru подразумевает, что вы прочитали и согласны с пользовательским соглашением

http://www.mathnet.ru/rus/agreement

Параметры загрузки:

IP : 35.173 .219 .12

26 апреля 2023 г., 08:26:15 
УДК $517.981 .1+517.518 .1+517.982 .3$

В.К. Захаров, А.В. Михалёв

\title{
Проблема общего радоновского представления для произвольного хаусдорфова пространства
}

\begin{abstract}
После основополагающих работ Рисса, Радона и Хаусдорфа 1909-1914 гг. стала актуальной проблема общего радоновского представления: для хаусдорфовых топологических пространств найти класс линейных функционалов, изоморфно интегрально представимых радоновскими мерами. K началу пятидесятых годов частичное решение проблемы (биективная версия) радоновского представления для локально компактных пространств было дано Халмошем, Хьюитом, Эдвардсом, Бурбаки и др. Для ограниченных радоновских мер на тихоновском пространстве проблема биективного представления была решена в 1956 г. Ю.В. Прохоровым.

В данной статье дается одно из возможных решений проблемы общего радоновского представления. Для этого используется семейство метаполунепрерывных функций с компактными носителями и класс тонких функционалов на нем. Найдены биективная и изоморфная версии решения (теоремы 1 и 2 из п. $5 \S 2$ ). Для получения изоморфной версии вводится семейство радоновских бимер.

Библиограффия: 38 наименований.
\end{abstract}

\section{Введение}

В 1909 г. Риссом [1] было доказано, что непрерывные линейные функционалы на пространстве непрерывных функций $C([0,1])$ на отрезке $[0,1]$ могут быть представлены интегралами Римана-Стилтьеса относительно функций ограниченной вариации на $[0,1]$. Теорема Рисса существенно использовала специфику пространства $[0,1]$, а именно: его линейную упорядоченность. Существенно новый шаг был сделан в 1913 г. Радоном [2], который для компактных подпространств $T \subset \mathbb{R}^{n}$ установил, что непрерывные линейные функционалы на пространстве $C(T)$ представляются интегралами относительно регулярных мер на $T$. Подход Радона, в котором центральное место занимает понятие регулярной меры, открыл перспективные пути для дальнейших обобшений.

В 1914 г. вышла книга Хаусдорфа [3], в которой были заложены основы теории топологических пространств. После этих работ стала актуальной проблема общего радоновского представления: для хаусдорфовых топологических пространств найти класс линейных функционалов, изоморфно интегрально представимых радоновскими (三 компактно регулярными) мерами. Более слабым вариантом этой проблемы стала проблема частного радоновского представления:

Авторы выражают благодарность ректору СПГУТД, академику, профессору В.Е. Романову и ректору МГУ, академику, профессору В.А. Садовничему за оказанное содействие совместному научному проекту двух университетов исследований по алгебре, топологии и теории меры, одним из результатов которого явилась эта работа.

(C) В.К. ЗАХАРов, А.В. МихАлёв, 1999 
для различных классов хаусдорфовых топологических пространств найти соответствующие классы функционалов, интегрально представимых радоновскими мерами.

Для компактных метрических пространств проблема радоновского представления была решена в 1937-38 гг. Банахом и Саксом [4], [5]. В 1941 г. Какутани [6] получил решение для произвольных компактных пространств.

$\mathrm{K}$ началу пятидесятых годов решение проблемы радоновского представления для локально компактных пространств было опубликовано Халмошем [7], Хьюитом [8], Эдвардсом [9], Бурбаки [10] и др. В результате появилась следующая замечательная теорема, получившая название первой теоремь Рисса-Радона о представлении (см., например, [11] и [12]).

Теорема. Пусть $(T, \mathscr{G})$ - локально компактное пространство и $C_{c}(T, \mathscr{G})$ решеточное векторное пространство всех непрерывных функиий на $(T, \mathscr{G})$ с компактными носителями. Тогда существует биекиия

$$
W:\left(C_{c}(T, \mathscr{G})^{\sim}\right)_{+} \longmapsto \mathfrak{R M}^{w o}(T, \mathscr{G})_{0}
$$

из множества всех положительных линейных функиионалов на $C_{c}(T, \mathscr{G})$ на множество всех положительных борелевско-радоновских мер на Т такая, ито $\varphi f=\int f d W \varphi$ для любой функиии $f \in C_{c}(T, \mathscr{G})$ и любого функиионала $\varphi \in\left(C_{c}(T, \mathscr{G})^{\sim}\right)_{+}$.

Для ограниченных радоновских мер на тихоновском пространстве проблема представления была решена в 1956 г. Ю. В. Прохоровым [13] с помошью введенного им класса компактно узких функционалов на решеточном векторном пространстве $C_{b}(T, \mathscr{G})$ (см. также [12]). Для неограниченных радоновских мер на тихоновском пространстве проблема оставалась открытой.

В [14], [15] Поллард и Топсое применили теорему типа Даниэля-Стоуна для рассмотрения радоновского представления на произвольном хаусдорфовом пространстве (однако ограничившись конусом (возможно, пустым) положительных непрерывных функций). В [16] Топсое перешел от использования пространства непрерывных функций к использованию конуса положительных полунепрерывных сверху функций. Это позволило Топсое [16] и Ангеру и Портенье [17] получить достаточные условия для представления функционалов радоновскими мерами.

На этом пути Кёниг [19], [20] получил биективную версию радоновского представления для положительных положительно-линейных функционалов на конусе положительных полунепрерывных сверху функций с компактными носителями.

Развивая методы Топсое и Фремлина [12], авторы в [21]-[23] получили линейно биективную и изоморфную версии общего радоновского представления.

В настоящей работе дается одно из возможных решений проблемы общего радоновского представления. Поскольку на произвольном хаусдорфовом пространстве семейство $C_{b}(T, \mathscr{G})$ может быть очень бедным, а семейство $C_{c}(T, \mathscr{G})$ может быть и вовсе тривиальным, используется семейство $S_{c}(T, \mathscr{G})$ всех метаполунепрерывны функиий с компактны.ми носителями, играющее для произвольных хаусдорфовых пространств ту же роль, которую для локально компактных пространств играет семейство $C_{c}(T, \mathscr{G})$. С решеточным векторным пространством $S_{c}(T, \mathscr{G})$ связывается решеточное векторное пространство $S_{c}(T, \mathscr{G})^{\triangle}$ всех тонких линейных функционалов. В итоге доказывается следующая теорема. 
ТеОрема 1 (см. п. $5 \S 2)$. Пусть $(T, \mathscr{G})$ - хаусдорфово пространство. Тогда существует биекиия

$$
V:\left(S_{c}(T, \mathscr{G})^{\triangle}\right)_{+} \longmapsto \mathfrak{R M}^{w o}(T, \mathscr{G})_{0}
$$

из множества всех тонких положительных линейных функиионалов на $S_{c}(T, \mathscr{G})$ на множество всех положительных борелевско-радоновских мер на $T$ такая, что $\varphi f=\int f d V \varphi$ для любой функиии $f \in S_{c}(T, \mathscr{G})$ и любого функиионала $\varphi \in\left(S_{c}(T, \mathscr{G})^{\triangle}\right)_{+}$.

Приведенные теоремы представляют собой биективные версии решения проблемы радоновского представления. Однако семейства $C_{c}(T, \mathscr{G})^{\sim}$ и $S_{c}(T, \mathscr{G})^{\triangle}$ являются решеточными векторными пространствами. Поэтому возникает задача поиска изоморфных версий решения проблемы общего радоновского представления.

Для получения изоморфных версий вводится семейство $\mathfrak{R} \mathfrak{B}(T, \mathscr{G})$ всех радоновских бимер на $T$ и строится биекция $E: \mathfrak{R M}^{w o}(T, \mathscr{G})_{0} \longmapsto \mathfrak{R B}(T, \mathscr{G})_{+}$такая, что любая бимера $\mathfrak{m} \in \mathfrak{R} \mathfrak{B}(T, \mathscr{G})$ представима в виде $\mathfrak{m}=E \mu^{\prime}-E \mu^{\prime \prime}$ для некоторых радоновских мер $\mu^{\prime}, \mu^{\prime \prime} \in \mathfrak{R M}^{w o}(T, \mathscr{G})_{0}$. Для изучения семейства бимер $\mathfrak{R} \mathfrak{B}(T, \mathscr{G})$ рассматривается сопутствуюшее решеточное векторное пространство узких радоновских мер. Это позволяет доказать, что семейство $\mathfrak{R} \mathfrak{B}(T, \mathscr{G})$ радоновских бимер является решеточным векторным пространством (теорема 1 из п. $2 \oint 2$ ). Далее определяется интеграл Лебега $\int f d \mathfrak{m}$ относительно бимеры m и устанавливается, что вложение $E$ сохраняет интеграл. В итоге доказываются следуюшие теоремы.

Теорема 2 (см. п. $5 \S 2)$. Пусть $(T, \mathscr{G})$ - хаусдорфово пространство. Тогда существует единственное продолжение биекции

$$
E \circ V:\left(S_{c}(T, \mathscr{G})^{\triangle}\right)_{+} \longmapsto \mathfrak{R B}(T, \mathscr{G})_{+}
$$

до изоморфизма $\mathfrak{I}: S_{c}(T, \mathscr{G})^{\triangle} \longmapsto \mathfrak{R B}(T, \mathscr{G})$ межуду данными решеточными векторнылми пространствами такое, что $\varphi f=\int f d \mathfrak{I} \varphi$ для любой функиии $f \in S_{c}(T, \mathscr{G})$ и любого функиионала $\varphi \in S_{c}(T, \mathscr{G})^{\triangle}$.

ТЕОРЕма 4 (см. П. $5 \S 2)$. Пусть $(T, \mathscr{G})$ - локально компактное пространство. Тогда существует единственное продолжение биекции

$$
E \circ W:\left(C_{c}(T, \mathscr{G})^{\sim}\right)_{+} \longmapsto \mathfrak{R B}(T, \mathscr{G})_{+}
$$

до изоморфизма $\mathfrak{J}: C_{c}(T, \mathscr{G})^{\sim} \longmapsto \mathfrak{R} \mathfrak{B}(T, \mathscr{G})$ между даннымми решеточнымми векторными пространствами такого, что $\varphi f=\int f d \mathfrak{J} \varphi$ для любой функиии $f \in C_{c}(T, \mathscr{G})$ и любого функиионала $\varphi \in C_{c}(T, \mathscr{G})^{\sim}$.

Частично результаты в этом направлении исследований были опубликованы в [21]-[23]. В данной работе дается подробное их изложение. Работа разбита на два параграффа. $\S 1$ носит вспомогательный вводный характер. Доказательства основных результатов помешены в $\S 2$. 


\section{§ 1. Предварительные сведения}

В этом параграфе мы приведем определения и утверждения, необходимые для основного § 2. Отметим, что изложение ведется в аксиоматике Неймана-БернайсаГёделя.

1. Меры и интеграл Лебега. Пусть $T$ - некоторое фиксированное множество, $\mathscr{P}(T)$ - множество всех подмножеств множества $T$ и $\mathscr{S}$ - некоторый ансамбль подмножеств множества $T$, т.е. $\mathscr{S} \subset \mathscr{P}(T)$. Любое отображение $\varepsilon: \mathscr{S} \rightarrow \overline{\mathbb{R}}$ будет называться оцениванием на $\mathscr{S}$. Оценивание $\varepsilon$ называется ограниченныцм, если $\operatorname{rng} \varepsilon \subset[a, b] \subset \mathbb{R}$ для некоторых $a, b \in \mathbb{R}$. Оценивание $\varepsilon$ называется положительным, если $\operatorname{rng} \varepsilon \subset \overline{\mathbb{R}}_{+}=[0, \infty]$. Оценивание $\varepsilon$ называется конечныц, если $\operatorname{rng} \varepsilon \subset \mathbb{R}$.

С оцениванием $\varepsilon$ связаны подмножества $\mathscr{S}^{0}(\varepsilon) \equiv\{S \in \mathscr{S} \mid \varepsilon S=0\}$ и $\operatorname{dom}_{f} \varepsilon \equiv$ $\mathscr{S}^{f}(\varepsilon) \equiv\{S \in \mathscr{S} \mid \varepsilon S \in \mathbb{R}\}$.

Оценивание $\varepsilon$ будет называться натуральным если:

1) $\varnothing \in \mathscr{S}$

2) $\varepsilon(\varnothing)=0$;

3) или $\operatorname{rng} \varepsilon \subset[-\infty, \infty[$, или $\operatorname{rng} \varepsilon \subset]-\infty, \infty]$.

Натуральное оценивание $\varepsilon$ называется $\sigma$-аддитивны.м, если

$$
\varepsilon S=\lim \left(\sum\left(\varepsilon S_{n} \mid n \in\{u(i) \in N \mid i \in \mathbb{N} \cap(k+1)\}\right) \mid k \in \mathbb{N}\right)
$$

для любого бесконечного подмножества $N \subset \omega \equiv\{0,1,2, \ldots\}$ и любой последовательности $\left(S_{n} \in \mathscr{S} \mid n \in N\right)$ попарно дизъюнктных элементов такой, что $S \equiv \cup\left\{S_{n} \mid n \in N\right\} \in \mathscr{S}$, где $u: \mathbb{N} \nrightarrow N$ - единственная изотонная биекция из множества $\mathbb{N} \equiv\{1,2, \ldots\}$ на множество $N$.

Натуральное $\sigma$-аддитивное оценивание $\mu: \mathscr{R} \rightarrow \overline{\mathbb{R}}$, определенное на кольце $\mathscr{R}$, называется мерой на $T$. Мера $\mu$ будет называться широкой, если $\mathscr{R}$ является $\sigma$-алгеброй.

Пусть $\mu: \mathscr{M} \rightarrow \overline{\mathbb{R}}_{+}-$широкая положительная мера. Через $F(T)$ обозначим семейство всех функций $f: T \rightarrow \mathbb{R}$, а через $M(T, \mathscr{M})$ - подсемейство всех $\mathscr{M}$-измеримых функций $f$ таких, что $f^{-1}[] x, y[] \in \mathscr{M}$ для любого интервала $] x, y[\subset \mathbb{R}$. Для $f \in F(T)_{+}$число $\sup \left\{\sum\left(\left(\inf f\left[M_{i}\right]\right) \mu M_{i} \mid i \in I\right) \mid\left(M_{i} \in \mathscr{M} \mid i \in I\right)\right.$ - конечное разбиение множества $T\} \in \overline{\mathbb{R}}_{+}$называется интегралом (Лебега) от функции $f$ относительно меры $\mu$ и обозначается через $\int f d \mu$ (см. [11, п. 12.2]). Семейство всех функций $f \in M(T, \mathscr{M})$ таких, что $\int f_{+} d \mu<\infty$ и $\int\left(-f_{-}\right) d \mu<\infty$, обозначим через $\mathrm{MI}(T, \mathscr{M}, \mu)$. Для $f \in \mathrm{MI}(T, \mathscr{M}, \mu)$ число

$$
\int f d \mu \equiv \int f_{+} d \mu-\int\left(-f_{-}\right) d \mu
$$

называется интегралом (Лебега) от функиии $f$ относительно меры $\mu$ (см. [11, п. 12.2]).

Семейство всех мер $\mu: \mathscr{R} \rightarrow \overline{\mathbb{R}}$ на кольце $\mathscr{R}$ обозначим через $\operatorname{Meas}(T, \mathscr{R})$. Его подсемейство всех положительных мер $\mu: \mathscr{R} \rightarrow \overline{\mathbb{R}}_{+}$обозначим через $\operatorname{Meas}(T, \mathscr{R})_{+}$. Для обозначения соответствуюших подсемейств ограниченных мер и конечных мер будем использовать символы $\operatorname{Meas}_{b}(T, \mathscr{R})$ и $\operatorname{Meas}_{f}(T, \mathscr{R})$. 
Teopema. 1) Ec $л u \mu, \nu \in \operatorname{Meas}(T, \mathscr{R})_{+} u \quad x, y \in \mathbb{R}_{+}, m o x \mu+y \nu \in \operatorname{Meas}(T, \mathscr{R})_{+}$.

2) Семейство $\operatorname{Meas}_{b}(T, \mathscr{R})$ является дедекиндово полным решеточным. векторныцм пространством.

3) $Е с л и \mathscr{R}$ является $\delta$-кольцом, то семейство $\operatorname{Meas}_{f}(T, \mathscr{R})$ является дедекиндово полным решеточным векторным пространством.

Для $\mu \in \operatorname{Meas}_{b}(T, \mathscr{R})$ рассмотрим $\mu_{+} \equiv \mu \vee o, \mu_{-} \equiv \mu \wedge o$ и $|\mu| \equiv \mu_{+} \vee\left(-\mu_{-}\right)$. Тогда $\mu=\mu_{+}+\mu_{-}$и $|\mu|=\mu_{+}-\mu_{-}$. Число $|\mu|(T)$ называется нормой меры $\mu$ и обозначается через $\|\mu\|$. Система $\left(\operatorname{Meas}_{b}(T, \mathscr{R}),\|\cdot\|\right)$ является нормированным решеточным векторным пространством с аддитивной нормой.

Пусть $\mu: \mathscr{M} \rightarrow \mathbb{R}$ - широкая ограниченная мера. Для нее рассмотрим дедекиндово полное решеточное векторное пространство

$$
\operatorname{MI}(T, \mathscr{M}, \mu) \equiv \operatorname{MI}\left(T, \mathscr{M}, \mu_{+}\right) \cap \operatorname{MI}\left(T, \mathscr{M},-_{-}\right) .
$$

Для $f \in \mathrm{MI}(T, \mathscr{M}, \mu)$ число

$$
\int f d \mu \equiv \int f d\left(\mu_{+}\right)-\int f d\left(-\mu_{-}\right)
$$

называется интегралом (Лебега) от функции $f$ относительно меры $\mu$ (см. [11, п. 19.17]).

Теорема. 1) Пусть $\mu, \nu$ - положительные меры на $\sigma$-алгебре $\mathscr{M} и x, y \in \mathbb{R}_{+}$. Тогда

$$
\begin{aligned}
\operatorname{MI}(T, \mathscr{M}, x \mu+y \nu) & =\mathrm{MI}(T, \mathscr{M}, \mu) \cap \mathrm{MI}(T, \mathscr{M}, \nu), \\
\int f d(x \mu+y \nu) & =x \int f d \mu+y \int f d \nu
\end{aligned}
$$

для любого $f \in \operatorname{MI}(T, \mathscr{M}, x \mu+y \nu)$.

2) Пусть $\mu, \nu$ - ограниченные меры на $\sigma$-алгебре $\mathscr{M} u x, y \in \mathbb{R}$. Если $f \in \operatorname{MI}(T, \mathscr{M}, \mu) \cap \operatorname{MI}(T, \mathscr{M}, \nu)$, mo $f \in \operatorname{MI}(T, \mathscr{M}, x \mu+y \nu) u \int f d(x \mu+y \nu)=$ $x \int f d \mu+y \int f d \nu$.

2. Некоторые классы функционалов. Далее $A(T)$ будет обозначать некоторое решеточное векторное подпространство в $F(T)$. Через $A(T)^{\sim}$ обозначается множество всех линейных функционалов $\varphi: A(T) \rightarrow \mathbb{R}$ таких, что $\varphi=\varphi_{1}-\varphi_{2}$ для некоторых линейных положительных функционалов $\varphi_{1}$ и $\varphi_{2}$. Будем называть такие функционалы бинарно-положительными.

Теорема (Рисса-Канторовича). $A(T)^{\sim}$ является дедекиндово полным решеточныц векторным пространством.

Для $\varphi \in A(T)^{\sim}$ рассмотрим $\varphi_{+} \equiv \varphi \vee 0, \varphi_{-} \equiv \varphi \wedge 0$ и $|\varphi| \equiv \varphi_{+} \wedge\left(-\varphi_{-}\right)$. Тогда $\varphi=\varphi_{+}+\varphi_{-}$и $|\varphi|=\varphi_{+}-\varphi_{-}$.

Если $A(T) \subset F_{b}(T) \equiv\left\{f \in F(T) \mid \exists x \in \mathbb{R}_{+}(|f| \leqslant x \mathbf{1})\right\}$, то на $A(T)$ рассматривается норма $\|f\|_{u} \equiv \sup \{|f(t)| \mid t \in T\}$. Семейство всех непрерывных линейных функционалов на нормированном векторном пространстве $\left(A(T),\|\cdot\|_{u}\right)$ обозначим через $\left(A(T),\|\cdot\|_{u}\right)^{\prime}$. 
TЕоремА. Если $A(T) \subset F_{b}(T)$, mo $\left(A(T),\|\cdot\|_{u}\right)^{\prime}$ является l-идеалом в $A(T)^{\sim}$ и, следовательно, $\left(A(T),\|\cdot\|_{u}\right)^{\prime}$ является дедекиндово полным решеточным векторным пространством. Относительно дуальной нормы $((A(T), \|$. $\left.\| u)^{\prime},\|\cdot\|\right)$ является нормированным решеточным векторным пространством.

Доказательство этих теорем можно найти в [24].

Пусть $f \in F(T)$ и $\left(f_{n} \in F(T) \mid n \in N \subset \omega\right)$ - бесконечная последовательность. Используем обозначение $f=p-\lim \left(f_{n} \mid n \in N\right)$, если $f(t)=\lim \left(f_{n}(t) \mid n \in N\right)$ для любого $t \in T$. Фукционал $\varphi: A(T) \rightarrow \mathbb{R}$ назовем поточечно $\sigma$-непрерывнылм, если $f=p-\lim \left(f_{n} \mid n \in N \subset \omega\right)$ влечет $\varphi f=\lim \left(\varphi f_{n} \mid n \in N\right)$ для каждой бесконечной последовательности $\left(f_{n} \in A(T) \mid n \in N \subset \omega\right)$ и каждой функции $f \in A(T)$. Множество всех поточечно $\sigma$-непрерывных функционалов на $A(T)$ обозначим через $A(T)^{\wedge}$. Если $A(T) \subset F_{b}(T)$, то множество $A(T)^{\wedge} \cap\left(A(T),\|\cdot\|_{u}\right)^{\prime}$ обозначим через $A(T) \otimes$.

Теорема. 1) $A(T)^{\wedge}$ является $l$-идеалом в $A(T)^{\sim}$ и, следовательно, является дедекиндово полным решеточным векторным пространством.

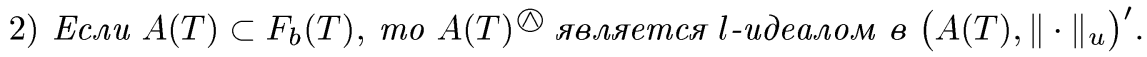

3. Продолжение некоторых оцениваний до мер. Пусть $\mathscr{K}$ и $\mathscr{L}$-некоторые ансамбли подмножеств множества $T$. Оценивание $\varepsilon: \mathscr{S} \rightarrow \overline{\mathbb{R}}$ назовем внутренне $\mathscr{K}$-регулярным, если $\mathscr{K} \subset \mathscr{S}$ и $\varepsilon S=\sup \left\{\varepsilon K \mid\left(K \in \mathscr{K} \cap \mathscr{S}^{f}(\varepsilon)\right) \wedge(K \subset S)\right\}$ для каждого $S \in \mathscr{S}$. Оценивание $\varepsilon$ называется верхне $\mathscr{K}$ - $\sigma$-непрерывным, если $\mathscr{K} \subset \mathscr{S}$ и $\left(\varepsilon K_{n} \mid n \in N\right) \downarrow \varepsilon K$ для каждого множества $K \in \mathscr{K}$ и каждой бесконечной последовательности $\left(K_{n} \in \mathscr{K} \mid n \in N \subset \omega\right) \downarrow K$ в $\mathscr{P}(T)$.

Насыщением ансамбля $\mathscr{S} \subset \mathscr{P}(T)$ назовем ансамбль

$$
\mathscr{U}(T, \mathscr{S}) \equiv\{U \in \mathscr{P}(T) \mid \forall S \in \mathscr{S}(U \cap S \in \mathscr{S})\}
$$

Если $\mathscr{S}=\mathscr{U}(T, \mathscr{S})$, то ансамбль $\mathscr{S}$ назовем насыщенным. Ансамбль $\mathscr{S}$ назовем $\mathscr{K}$-насыщенным, если $\mathscr{K} \subset \mathscr{S}$ и условия $U \in \mathscr{P}(T)$ и $U \cap K \in \mathscr{S}$ для каждого $K \in \mathscr{K}$ влекут $U \in \mathscr{S}$.

Для оценивания $\varepsilon: \mathscr{S} \rightarrow \overline{\mathbb{R}}$ рассмотрим ансамбли

$$
\begin{aligned}
\mathscr{N}_{0}(T, \mathscr{S}, \varepsilon) & \equiv\left\{N_{0} \in \mathscr{U}(T, \mathscr{S}) \mid \forall S \in \mathscr{S}\left(\left(S \subset N_{0}\right) \Rightarrow(\varepsilon S=0)\right)\right\} \\
& =\left\{N_{0} \in \mathscr{U}(T, S) \mid \forall S \in \mathscr{S}\left(\varepsilon\left(N_{0} \cap S\right)=0\right)\right\}
\end{aligned}
$$

и

$$
\mathscr{N}(T, \mathscr{S}, \varepsilon) \equiv\left\{N \subset T \mid \exists N_{0} \in \mathscr{N}_{0}(T, \mathscr{S}, \varepsilon)\left(N \subset N_{0}\right)\right\}
$$

Оценивание $\varepsilon$ называется полныл, если $\mathscr{N}(T, \mathscr{S}, \varepsilon) \subset \mathscr{S}$ (см. [25]). Для неполного оценивания $\varepsilon$ рассмотрим ансамбль $\mathscr{K}(T, \mathscr{S}, \varepsilon) \equiv\{S \cup N \mid S \in \mathscr{S} \wedge N \in \mathscr{N}(T, \mathscr{S}, \varepsilon)\}$ и оценивание $\stackrel{\vee}{\varepsilon}: \mathscr{K}(T, \mathscr{S}, \varepsilon) \rightarrow \overline{\mathbb{R}}$ такое, что $\stackrel{\vee}{\varepsilon}(S \cup N) \equiv \varepsilon S$. 
ТеОрема. Пусть $\mu: \mathscr{R} \rightarrow \overline{\mathbb{R}}-$ мера на кольце $\mathscr{R}$. Тогда $\mathscr{K}(T, \mathscr{R}, \mu)$ является кольиом, содержащим кольцо $\mathscr{R}$, и $\underset{\vee}{\mu}$ является полной мерой, продолжсающей меру н. Если $\mathscr{R}-\delta$-кольио, то $\mathscr{K}(T, \mathscr{R}, \mu)$ тоже $\delta$-кольцо.

Согласно [25] мера $\stackrel{\vee}{\mu}$ назьвается пополнением меры $\mu$.

Для оценивания $\varepsilon: \mathscr{S} \rightarrow \overline{\mathbb{R}}$ ансамбль $\mathscr{U}_{s}(T, \mathscr{S}, \varepsilon) \equiv\{U \in \mathscr{P}(T) \mid \forall S \in$ $\left.\mathscr{S}^{f}(\varepsilon)(U \cap S \in \mathscr{S})\right\}$ назовем сильным насышением ансамбля $\mathscr{S}$ относительно $\varepsilon$. Если $\mathscr{S}=\mathscr{U}_{s}(T, \mathscr{S}, \varepsilon)$, то оценивание $\varepsilon$ назовем сильно насыщенным.

Положительное оценивание $\varepsilon: \mathscr{S} \rightarrow \overline{\mathbb{R}}_{+}$назовем внутренне конечным, если для каждого $S \in \mathscr{S}$ такого, что $\varepsilon S=\infty$, существует $R \in \mathscr{S}$ такое, что $R \subset S$ и $0<\varepsilon R<\infty$. Для положительного оценивания $\varepsilon$ определим оценивание $\varepsilon^{\prime \prime}: \mathscr{U}_{s}(T, \mathscr{S}, \varepsilon) \rightarrow \overline{\mathbb{R}}_{+}$, полагая $\varepsilon^{\prime \prime} U \equiv \sup \left\{\varepsilon(U \cap S) \mid S \in \mathscr{S}^{f}(\varepsilon)\right\}$ для каждого $U \in \mathscr{U}_{s}(T, \mathscr{S}, \varepsilon)$.

ТЕОРеМА. Пусть $\mu: \mathscr{R} \rightarrow \overline{\mathbb{R}}_{+}-$положительная внутренне конечная мера на $\delta$-кольце $\mathscr{R}$. Тогда $\mu^{\prime \prime}$ является сильно насыщенной внутренне конечной мерой на б-алгебре $\mathscr{U}_{s}(T, \mathscr{R}, \mu)$, продолжающей меру $\mu$. Если $\mu$ является полной, то $\mu^{\prime \prime}$ тоже является полной.

Меру $\mu^{\prime \prime}$ назовем сильным насышением, а меру $\mu^{\#} \equiv(\stackrel{\vee}{\mu})^{\prime \prime}$ назовем полнылм сильным насыщением внутренне конечной положительной меры $\mu$, заданной на $\delta$-кольие $\mathscr{R}$.

Следуюшая теорема, доказательство которой приведено в $[12,71 \mathrm{~A}]$, является ключевым результатом для интегрального представления функционалов.

ТЕОРЕМА. Пусть $\mathscr{K}-$ аддитивный $\delta$-мультипликативный ансамбль на множестве $T$ и $\lambda: \mathscr{K} \rightarrow \mathbb{R}_{+}-$возрастающее натуральное оченивание такое, что:

1) $\lambda(H \cup K) \geqslant \lambda H+\lambda K$ для любых $H, K \in \mathscr{K}$ maкux, ито $H \cap K=\varnothing$;

2) $\lambda L \leqslant \lambda H+\sup \{\lambda K \mid(K \in \mathscr{K}) \wedge(K \subset L \backslash H)\}$ для любих $H, L \in \mathscr{K}$ mаких, что $H \subset L$;

3) $\left(\lambda K_{n} \mid n \in w\right) \downarrow 0$ для каждой последовательности $\left(K_{n} \in \mathscr{K} \mid n \in w\right) \downarrow \varnothing$ в $\mathscr{P}(T)$.

Определим оценивание $\nu: \mathscr{P}(T) \rightarrow \overline{\mathbb{R}}_{+}$, полагая $\nu E \equiv \sup \{\lambda K \mid(K \in \mathscr{K}) \wedge$ $(K \subset E)\}$. Рассмотрим ансамбль $\mathscr{M} \equiv\{M \subset T \mid \forall L \in \mathscr{K}(\lambda L \leqslant \nu(L \cap M)+$ $\nu(L \backslash M))\}$ и оченивание $\mu \equiv \nu \mid \mathscr{M}$. Тогда $\mathscr{M}$ является $\sigma$-алгеброй $u \mu: \mathscr{M} \rightarrow \overline{\mathbb{R}}_{+}$ является положстельной, полной, сильно насыщенной, внутренне $\mathscr{K}$-регулярной и Ж-насыщенной мерой, продолжсощей оценивание $\lambda$.

Обозначим множество всех оцениваний $\lambda: \mathscr{K} \rightarrow \mathbb{R}_{+}$на $T$, удовлетворяющих условиям этой теоремы, через $\mathfrak{L}(T, \mathscr{K})_{0}$, а множество всех положительных широких мер $\mu: \mathscr{M} \rightarrow \overline{\mathbb{R}}_{+}$на $T$ через $\mathfrak{M}(T)_{0}$. Для обозначения соответствующих подмножеств ограниченных оцениваний и мер будем использовать нижний индекс $b$. Согласно данной теореме мы можем корректно определить отображение $e(\mathscr{K}): \mathfrak{L}(T, \mathscr{K})_{0} \rightarrow \mathfrak{M}(T)_{0}$, полагая $e(\mathscr{K}) \lambda \equiv \mu$. Отображение $e(\mathscr{K})$ назовем расширяющим. 


\section{4. Теорема Александрова-Стоуна об интегральном представлении.} Если $\mu: \mathscr{M} \rightarrow \overline{\mathbb{R}}_{+}$является положительной мерой или $\mu: \mathscr{M} \rightarrow \mathbb{R}$ является ограниченной мерой на $\sigma$-алгебре $\mathscr{M}$, то согласно п. 1 мы можем определить на решеточном векторном пространстве $\mathrm{MI}(T, \mathscr{M}, \mu)$ функционал $\Lambda(\mu)$, полагая $\Lambda(\mu) f \equiv$ $\int f d \mu$ для любого $f \in \operatorname{MI}(T, \mathscr{M}, \mu)$. По теореме Лебега об ограниченной сходимости (см. [11, п. 12.24]) функционал $\Lambda(\mu)$ является поточечно $\sigma$-непрерывным.

Оказывается, что это свойство является не только необходимым, но и достаточным для интегрального представления функционалов.

Пусть $A(T)$ - функциональное семейство на множестве $T$, т.е. $A(T) \subset F(T)$. Оно называется усекаемым (三со свойством Стоуна), если $f \wedge \mathbf{1} \in A(T)$ для каждого $f \in A(T)$. Множество $K \subset T$ назовем $\sigma$-вписанным в семейство $A(T)$, если сушествует последовательность $\left(f_{n} \in A(T)_{+} \mid n \in N \subset \omega\right) \downarrow$ такая, что $\left(f_{n} \mid n \in N\right) \downarrow \chi(K)$ в $F(T)$ (т.е. поточечно). Ансамбль всех подмножеств из $T$, $\sigma$-вписанных в $A(T)$, обозначим через $\mathscr{J}_{\sigma}(A(T))$.

Лемма. Пусть $A(T)$ - усекаемое решеточное векторное подпространство в $F(T)$ u $f \in A(T)$. Тогда:

1) $f \wedge x \mathbf{1} \in A(T)$ для каждого $x>0$;

2) $S f \equiv\{t \in T \mid f(t) \geqslant 1\} \in \mathscr{J}_{\sigma}(A(T))$.

ПРЕДЛОЖЕНИЕ. Пусть $A(T)$ - усекаемое решеточное векторное пространство наТ. Тогда ансамбль $\mathscr{J}_{\sigma}(A(T))$ является аддитивныцм и $\delta$-мультипликативным.

Пусть $A(T)$ - решеточное векторное пространство на $T$ и $\varphi$ - линейный функционал на $A(T)$. Пусть $(T, \mathscr{M}, \mu)$ - измеримое пространство с мерой $\mu$ на $\sigma$-алгебре $\mathscr{M}$. Функционал $\varphi$ называется представимым интегралом Лебега над измеримымм пространством $(T, \mathscr{M}, \mu)$, или представимым в виде интеграла Лебега относительно меры $\mu$, если $A(T) \subset \mathrm{MI}(T, \mathscr{M}, \mu)$ и $\varphi=\Lambda(\mu) \mid A(T)$. Ясно, что такой функционал необходимо поточечно $\sigma$-непрерывен.

ПРЕДЛОЖЕНИЕ. Пусть $\mu$ - положительная мера на б-алгебре $\mathscr{M}$ на $Т$ и $A(T)$ - усекаемое решеточное векторное пространство на $T$ такое, что $S f \in \mathscr{M}^{f}(\mu)$ для каждого $f \in A(T)$. Пусть $\varphi-$ поточечно $\sigma$-непрерывный положительный линейный функционал на $A(T)$ такой, что $\mu(S f)=\inf \{\varphi g \mid$ $\left.\left(g \in A(T)_{+}\right) \wedge(g \geqslant \chi(S f))\right\}$. Тогда ч представляется интегралом Лебега над измеримым пространством $(T, \mathscr{M}, \mu)$.

Доказательство этого предложения приведено в $[12,71 \mathrm{~F}]$.

Следуюший важный результат может быть назван теоремой АлександроваСтоуна об интегральном представлении, поскольку он восходит к работам [26] и [27]. Стоун показал важность поточечной $\sigma$-непрерывности для существования меры $\mu$, а А. Д. Александров показал важность внутренней регулярности для единственности $\mu$.

Теорема (Александрова-Стоуна). Пусть $A(T)$ - усекаемое решеточное векторное пространство на $T$ и $\varphi \in\left(A(T)^{\wedge}\right)_{+}$. Определим на ансамбле $\mathscr{K} \equiv$ $\mathscr{J}_{\sigma}(A(T))$ оченивание $\lambda: \mathscr{K} \rightarrow \mathbb{R}_{+}$, полагая $\lambda K \equiv \inf \left\{\varphi f \mid\left(f \in A(T)_{+}\right) \wedge\right.$ $(f \geqslant \chi(K))\}$. Тогда: 
1) $\lambda \in \mathfrak{L}(T, \mathscr{K})$

2) мера $\mu \equiv е(\mathscr{K}) \lambda: \mathscr{M} \rightarrow \overline{\mathbb{R}}_{+}$из п. 3 является положительной, иирокой, полной, сильно насыщенной, $\mathscr{K}$-насыщенной, внутренне $\mathscr{K}$-регулярной и верхне $\mathscr{K}$ - - -непрерывной;

3) функционал $\varphi$ представим интегралом Лебега над измеримым пространством $(T, \mathscr{M}, \mu)$;

4) мера $\mu$ является единственной в следующем смысле: если существует другая положительная, полная, сильно насыщенная и внутренне ЖК-регулярная мера $\nu: \mathscr{N} \rightarrow \overline{\mathbb{R}}_{+}$такая, что функчионал $\varphi$ представим интегралом Лебега над пространством $(T, \mathcal{N}, \nu)$, то $\mathscr{N}=\mathscr{M}$ и $\nu=\mu$;

5) если $A(T) \subset F_{b}(T)$ и $\varphi \in\left(A(T)^{\otimes}\right)_{+}$, то мера $\mu$ является ограниченной.

Доказательство утверждений 1)-3) этой теоремы приведено в $[12,71 \mathrm{G}]$.

Из этой теоремы следует, что можно корректно определить отображение $r:\left(A(T)^{\wedge}\right)_{+} \rightarrow \mathfrak{L}\left(T, \mathscr{J}_{\sigma}(A(T))\right)_{0}$, полагая $r \varphi \equiv \lambda$. Более того, можно корректно определить отображение $V^{\wedge}:\left(A(T)^{\wedge}\right)_{+} \rightarrow \mathfrak{M}(T)_{0}$, полагая $V^{\wedge}(\varphi) \equiv \mu \equiv$ $e\left(\mathscr{J}_{\sigma}(A(T))\right)(r \varphi)$. Если $A(T) \subset F_{b}(T)$, то можно также определить отображение $V_{b}^{\wedge}:\left(A(T)^{\otimes}\right)_{+} \rightarrow \mathfrak{M}_{b}(T)_{+}$, полагая $V_{b}^{\wedge} \equiv V^{\wedge} \mid\left(A(T)^{\otimes}\right)_{+}$. Очевидно, что отображения $V^{\wedge}$ и $V_{b}^{\wedge}$ инъективны.

Если мы возьмем другой функционал $\psi \in\left(A(T)^{\wedge}\right)_{+}$, то по теореме Александрова-Стоуна для $\psi$ сушествует свое собственное измеримое пространство $(T, \mathcal{N}, \nu)$ такое, что $\varphi$ представим интегралом Лебега над $(T, \mathcal{N}, \nu)$. Поэтому важно рассмотреть для всех таких функционалов интегральное представление над одним и тем же измеримым пространством.

По этой причине для ансамбля $\mathscr{K} \equiv \mathscr{J}_{\sigma}(A(T))$ рассмотрим его борелевскую оболочку $\mathscr{B}(T, \mathscr{K})$ в $\mathscr{P}(T)$, т. е. наименьшую $\sigma$-алгебру в $\mathscr{P}(T)$, содержашую $\mathscr{K}$. Если $\varphi \in\left(A(T)^{\wedge}\right)_{+}, \mu \equiv V^{\wedge}(\varphi) \in \mathfrak{M}(T)_{0}$ и $\mathscr{M} \equiv \operatorname{dom} \mu$, то $\mathscr{K} \subset \mathscr{M}$ влечет $\mathscr{B}(T, \mathscr{K}) \subset \mathscr{M}$, поскольку $\mathscr{M}$ является $\sigma$-алгеброй.

В $\S 2$ мы рассмотрим произвольное решеточное векторное подпространство $A(T)^{\#}$ решеточного векторного пространства $A(T)^{\wedge}$ и соответствующее решеточное векторное подпространство $A(T)^{\boxplus} \equiv A(T)^{\#} \cap A(T)^{\otimes}$ в $A(T)^{\#}$ для $A(T) \subset F_{b}(T)$.

Возьмем любую $\sigma$-алгебру $\mathscr{M}_{0}$ такую, что

$$
\mathscr{B}(T, \mathscr{K}) \subset \mathscr{M}_{0} \subset \cap\left\{\operatorname{dom} V^{\wedge}(\varphi) \mid \varphi \in\left(A(T)^{\#}\right)_{+}\right\} .
$$

Тогда для каждого функционала $\varphi \in\left(A(T)^{\#}\right)_{+}$и для соответствующей меры $\mu \equiv V^{\wedge}(\varphi)$ мы можем рассмотреть меру $\mu_{0} \equiv \mu \mid \mathscr{M}_{0}$.

Это означает, что мы можем корректно определить отображение $V$ : $\left(A(T)^{\#}\right)_{+} \rightarrow \operatorname{Meas}\left(T, \mathscr{M}_{0}\right)_{+}$, полагая $V \varphi \equiv\left(V^{\wedge}(\varphi)\right) \mid \mathscr{M}_{0}$, и отображение $V_{b}:\left(A(T)^{\boxplus}\right)_{+} \rightarrow \operatorname{Meas}_{b}\left(T, \mathscr{M}_{0}\right)_{+}$, полагая $V_{b} \equiv V \mid\left(A(T)^{\boxplus}\right)_{+}$. Эти отображения инъективны.

Следуюшая теорема является непосредственным следствием из предыдущей.

Теорема. Пусть $A(T)$ - усекаемое решеточное векторное пространство на $T, \mathscr{K} \equiv \mathscr{J}_{\sigma}(A(T)), \quad A(T)^{\#}$ - решеточное векторное подпространство в $A(T)^{\wedge}, \varphi \in\left(A(T)^{\#}\right)_{+} u \mu_{0} \equiv V \varphi$. Тогда: 
1) $\mu_{0}$ является положительной, внутренне $\mathscr{K}$-регулярной и верхне $\mathscr{K}$-б-непрерывной мерой;

2) функиионал $\varphi$ представим интегралом Лебега над измеримым пространством $\left(T, \mathscr{M}_{0}, \mu_{0}\right)$;

3) мера $\mu_{0}$ единственна в следующем смьсле: если существует другая положительная, внутренне $\mathscr{K}$-регулярная мера $\varkappa: \mathscr{M}_{0} \rightarrow \overline{\mathbb{R}}_{+}$такая, что функиионал ч представим интегралом Лебега над $\left(T, \mathscr{M}_{0}, \varkappa\right)$, то $\varkappa=\mu_{0}$;

4) если $A(T) \subset F_{b}(T)$ и $\varphi\left(A(T)^{\boxplus}\right)_{+}$, то мера $\mu_{0}$ ограничена.

5. Широкие радоновские меры на хаусдорфовом топологическом пространстве. Далее $(T, \mathscr{G})$ будет обозначать хаусдорфово топологическое пространство с ансамблем $\mathscr{G}$ всех открытых подмножеств из $T$. Соответствуюшие ансамбли всех замкнутых и всех компактных подмножеств из $T$ обозначаем соответственно через $\mathscr{F}$ и $\mathscr{C}$. В силу хаусдорфовости $\mathscr{C} \subset \mathscr{F}$.

Оценивание $\varepsilon: \mathscr{S} \rightarrow \overline{\mathbb{R}}$ называется локально ограниченным, если для каждой точки $t \in T$ существует $G \in \mathscr{G}$ такое, что $t \in G$ и $\sup \{|\varepsilon S| \mid(S \in \mathscr{S}) \wedge$ $(S \subset G)\}<\infty$. Оценивание $\varepsilon$ называется (внутренне) компактно регулярным если $\varepsilon S=\sup \left\{\varepsilon C \mid\left(C \in \mathscr{C} \cap \mathscr{S}^{f}(\varepsilon)\right) \wedge(C \subset S)\right\}$ для каждого $S \in \mathscr{S}$.

Положительная мера $\mu: \mathscr{M} \rightarrow \overline{\mathbb{R}}_{+}$называется положительной (широкой) радоновской мерой (см. [12, 73A]; [10, X, § 3.2]; [28]), если она является компактно регулярной и локально ограниченной и $\mathscr{M}$ является $\sigma$-алгеброй, содержащей ансамбль $\mathscr{G}$. Ясно, что для такой меры $\sigma$-алгебра $\mathscr{M}$ содержит $\sigma$-алгебру $\mathscr{B}(T, \mathscr{G})$ всех борелевских подмножеств из $T$. Если $\mu$ является полной и сильно насышенной, то будем называть ее положительной (иирокой) расширенной радоновской мерой. Если $\mathscr{M}=\mathscr{B}(T, \mathscr{G})$, то $\mu$ будем называть положительной (иирокой) борелевско-радоновской мерой.

Семейство всех положительных (широких) радоновских мер $\mu: \mathscr{M} \rightarrow \overline{\mathbb{R}}_{+}$на хаусдорфовом топологическом пространстве $(T, \mathscr{G})$, определенных на всех возможных $\sigma$-алгебрах $\mathscr{M}$, обозначим через $\mathfrak{R M}^{w}(T, \mathscr{G})_{0}$. Его подсемейство, состояшее из всех мер, определенных на фиксированной $\sigma$-алгебре $\mathscr{M}$, обозначим через $\mathfrak{R M}^{w}(T, \mathscr{G}, \mathscr{M})_{0}$. Подсемейство в $\mathfrak{R M}^{w}(T, \mathscr{G})_{0}$, состоящее из всех расширенных мер, обозначим через $\mathfrak{R M} \mathfrak{M e}^{w}(T, \mathscr{G})_{0}$, а подсемейство, состоящее из всех борелевско-радоновских мер, обозначим через $\mathfrak{R M} \mathfrak{M}^{w o}(T, \mathscr{G})_{0}$. Для обозначения соответствующих подсемейств ограниченных мер будем использовать нижний индекс $b$.

Ограниченная мера $\mu: \mathscr{M} \rightarrow \mathbb{R}$, определенная на $(T, \mathscr{G})$, называется бинарно-положительной (широкой) ограниченной радоновской мерой, если $\mu=$ $\mu_{1}-\mu_{2}$ для некоторых положительных (широких) ограниченных радоновских мер $\mu_{1}: \mathscr{M} \rightarrow \mathbb{R}_{+}$и $\mu_{2}: \mathscr{M} \rightarrow \mathbb{R}_{+}$. Если $\mu_{1}, \mu_{2} \in \mathfrak{R M}_{b}^{w o}(T, \mathscr{G})_{0}$, то $\mu$ будем называть бинарно-положительной (широкой) ограниченной борелевско-радоновской мерой.

Семейство всех бинарно-положительньх (широких) ограниченных радоновских мер на $(T, \mathscr{G})$, определенных на всех возможных $\sigma$-алгебрах $\mathscr{M}$, обозначим через $\mathfrak{R M}_{b}^{w}(T, \mathscr{G})$. Его подсемейство, состоящее из всех борелевско-радоновских мер, обозначим через $\mathfrak{R M} w b(T, \mathscr{G})$, а подсемейство, состоящее из всех радоновских мер, определенных на фиксированной $\sigma$-алгебре $\mathscr{M}$, обозначим через $\mathfrak{R M}_{b}^{w}(T, \mathscr{G}, \mathscr{M})$.

Заметим, что Шварц [28] рассматривает только радоновские меры из $\mathfrak{R M}^{w o}(T, \mathscr{G})_{0}$, а $\Phi$ ремлин [12] рассматривает только радоновские меры 
из $\mathfrak{R M}^{w e}(T, \mathscr{G})_{0}$. Однако сушествует следующее биективное соответствие между этими классами радоновских мер.

Лемма. Пусть $\mu \in \mathfrak{R M}^{w}(T, \mathscr{G})_{0} . \quad$ Тогда ограничение $\mu_{0} \equiv \mu \mid \mathscr{B}(T, \mathscr{G})$ является борелевско-радоновской мерой, а расширения $\mu^{\#} u\left(\mu_{0}\right)^{\#}$ из $n .3$ являются расширенными радоновскими мерами и $\mu^{\#}=\left(\mu_{0}\right)^{\#}$. Более того, $\mu^{\#}$ является единственным положительным, широким, полньм и сильно насыщенным расширением мер $\mu$ и $\mu_{0}$ среди всех положстельных широких радоновских мер $\nu: \mathcal{N} \rightarrow \overline{\mathbb{R}}_{+}$на $(T, \mathscr{G})$, продолэсающих меру $\mu_{0}$. Все три данные меры $\mu, \mu_{0} и \mu^{\#}$ являются ограниченными одновременно. Если мера $\mu$ является $\sigma$-конечной, то $\mu^{\#}$ является тоже $\sigma$-конечной.

Отметим также, что понятие произвольной (широкой) радоновской меры $\mu$ : $\mathscr{M} \rightarrow \overline{\mathbb{R}}$ не определено.

ПРЕДЛОЖЕНИЕ. 1) $Е с л u \mu, \nu \in \mathfrak{R M}^{w}(T, \mathscr{G}, \mathscr{M})_{0} u x, y \in \mathbb{R}_{+}$, mo $x \mu+y \nu \in$ $\mathfrak{R M}^{w}(T, \mathscr{G}, \mathscr{M})_{0}$.

2) $\mathfrak{R M}_{b}^{w}(T, \mathscr{G}, \mathscr{M})$ является l-идеалом в $\operatorname{Meas}_{b}(T, \mathscr{M})$ и, следовательно, является дедекиндово полным решеточным векторным пространством.

3) $\mathfrak{R M}_{b}^{w}(T, \mathscr{G}, \mathscr{M})_{+}=\mathfrak{R M}_{b}^{w}(T, \mathscr{G}, \mathscr{M})_{0}$.

Из утверждения 3) этого предложения следует, что мы можем опустить слова "бинарно-положительньй" и называть бинарно-положительную (широкую) ограниченную радоновскую меру просто (иирокой) ограниченной радоновской мерой.

Относительно нормы $\|\cdot\|$, индуцированной из $\left(\operatorname{Meas}_{b}(T, \mathscr{M}),\|\cdot\|\right)$, система $\left(\mathfrak{R M}_{b}^{w}(T, \mathscr{G}, \mathscr{M}),\|\cdot\|\right)$ является нормированным решеточным векторным пространством.

6. Вторая теорема Рисса-Радона о представлении для локально компактных пространств. Первая теорема Рисса-Радона о представлении была приведена во введении. Для ограниченных радоновских мер на локально компактном пространстве справедлива также изоморфная версия решения проблемы радоновского представления, получившая название второй теоремы Puсcа-Радона о представлении.

ТЕОРемА. Пусть $(T, \mathscr{G})$ - локально компактное пространство $и \mathrm{~W}-$ биекция из первой теоремы Рисса-Радона о представлении. Тогда существует единственное продолэение биекции

$$
W_{b} \equiv W \mid\left(C_{c}(T, \mathscr{G}),\|\cdot\|_{u}\right)_{+}^{\prime}:\left(C_{c}(T, \mathscr{G}),\|\cdot\|_{u}\right)^{\prime} \longmapsto \mathfrak{R M}_{b}^{w o}(T, \mathscr{G})_{0}
$$

до (изометрического) изоморфизма

$$
J_{b}:\left(\left(C_{c}(T, \mathscr{G}),\|\cdot\|_{u}\right)^{\prime},\|\cdot\|\right) \longmapsto\left(\mathfrak{R M}_{b}^{w o}(T, \mathscr{G}),\|\cdot\|\right)
$$

данных нормированных решеточных векторных пространств такого, что $\varphi f=\int f d J_{b} \varphi$ для любой функиии $f \in C_{c}(T, \mathscr{G})$ и любого функционала $\varphi \in$ $\left(C_{c}(T, \mathscr{G}),\|\cdot\|_{u}\right)^{\prime}$.

Доказательство этой теоремы (в несколько другой формулировке) можно найти в $[11]$. 
7. Теорема Прохорова о представлении для ограниченных радоновских мер на тихоновских пространствах. Важное свойство интеграла Лебега над топологическим измеримым пространством $(T, \mathscr{G}, \mathscr{M}, \mu)$ с радоновской мерой $\mu$ было найдено Прохоровым в работе [13].

Пусть $A(T)$ является функциональным решеточным векторным пространством на топологическом пространстве $(T, \mathscr{G})$. Функционал $\varphi: A(T) \rightarrow \mathbb{R}$ называется (компактно) узким (или со свойством Прохорова), если для каждого $\varepsilon>0$ существует компактное подмножество $K \in \mathscr{C}$ такое, что условия $f \in A(T)$ и $|f| \leqslant \chi(T \backslash K)$ влекут $|\varphi f|<\varepsilon$. Линейный функционал $\varphi: A(T) \rightarrow \mathbb{R}$ называется бинарно-положительным (компактно) узким, если $\varphi=\varphi_{1}-\varphi_{2}$ для некоторых положительных компактно узких линейных функционалов $\varphi_{1}$ и $\varphi_{2}$. Ясно, что $\varphi$ является также компактно узким. Семейство всех положительных (компактно) узких линейных функционалов на $A(T)$ обозначим через $\left(A(T)^{\pi}\right)_{0}$. Семейство всех бинарно-положительных (компактно) узких линейных функционалов на $A(T)$ обозначим через $A(T)^{\pi}$. Если $A(T) \subset F_{b}(T)$, то семейство $A(T)^{\pi} \cap\left(A(T),\|\cdot\|_{u}\right)^{\prime}$ обозначаем через $A(T)^{\circledR \pi}$.

ЛЕмма. 1) $A(T)^{\pi}$ является l-идеалом в $A(T)^{\sim}$ и, следовательно, является дедекиндово полным решеточным векторным пространством $и\left(A(T)^{\pi}\right)_{+}=$ $\left(A(T)^{\pi}\right)_{0}$.

2) Если $A(T) \subset F_{b}(T)$, то $A(T)^{\pi}$ является l-идеалом в $\left(A(T),\|\cdot\|_{u}\right)^{\prime}$.

Из равенства $\left(A(T)^{\pi}\right)_{+}=\left(A(T)^{\pi}\right)_{0}$ следует, что мы можем опустить слова "бинарно-положительный" и называть бинарно-положительный (компактно) узкий линейный функционал просто (компактно) узким линейным функционалом.

Относительно нормы $\|\cdot\|$, индуцированной из $\left(\left(A(T),\|\cdot\|_{u}\right)^{\prime},\|\cdot\|\right)$, система $\left(A(T)^{\mathbb{\pi}},\|\cdot\|\right)$ является нормированным решеточным векторным пространством.

В работе [13] было дано следуюшее решение проблемы радоновского представления (см. также [10]).

Теорема (Прохорова о представлении). Пусть $(T, \mathscr{G})$ - тихоновское топологическое пространство и $C_{b}(T, \mathscr{G})$ - решеточное векторное пространство всех ограниченных непрерывных функций на $(T, \mathscr{G})$. Тогда существует (изометрический) изоморфизм $J_{b}:\left(C_{b}(T, \mathscr{G})^{\mathbb{\pi}},\|\cdot\|\right) \longmapsto\left(\mathfrak{R M}_{b}^{w o}(T, \mathscr{G}),\|\cdot\|\right)$ данных нормированных решеточных векторных пространств такой, что $\varphi f=$ $\int f d J_{b} \varphi$ для любой функиии $f \in C_{b}(T, \mathscr{G})$ и любого функиионала $\varphi \in C_{b}(T, \mathscr{G})^{\mathbb{\pi}}$.

Для неограниченных радоновских мер на тихоновском пространстве проблема представления оставалась открытой.

\section{§ 2. Основная часть}

В этом параграфе мы дадим подробное изложение решения проблемы общего радоновского представления. Далее $(T, \mathscr{G})$ будет обозначать произвольное хаусдорфово топологическое пространство.

1. Узкие радоновские меры. Поскольку семейство $\mathfrak{R} \mathfrak{M}^{w o}(T, \mathscr{G})_{0}$ не является векторным пространством, мы рассмотрим сначала важное решеточное векторное пространство, связанное с этим семейством. 
Напомним, что подмножество $P$ из T называется предкомпактным (三 относительно компактным), если сl $P$ является компактным, т.е. $P$ содержится в некотором компактном множестве. Подансамбль ансамбля $\mathscr{B}(T, \mathscr{G})$, состоящий из всех предкомпактных борелевских множеств, обозначим через $\mathscr{B}_{c}(T, \mathscr{G})$. Он является $\delta$-кольцом.

Конечную положительную меру $\mu: \mathscr{M} \rightarrow \mathbb{R}_{+}$на пространстве $(T, \mathscr{G})$ назовем положительной узкой радоновской мерой, если $\mu$ является компактно регулярной и локально ограниченной, а $\mathscr{M}$ является $\delta$-кольцом, содержащим $\delta$-кольцо $\mathscr{B}_{c}(T, \mathscr{G})$ и таким, что $M \cap G \in \mathscr{M}$ для каждых $M \in \mathscr{M}$ и $G \in \mathscr{G}$. Если $\mathscr{M}=\mathscr{B}_{c}(T, \mathscr{G})$, то $\mu$ назовем положительной узкой борелевско-радоновской мерой.

Семейство всех положительных узких радоновских мер $\mu: \mathscr{M} \rightarrow \mathbb{R}_{+}$на пространстве $(T, \mathscr{G})$, определенных на всех возможных $\delta$-кольцах $\mathscr{M}$, обозначим через $\mathfrak{R M}^{n}(T, \mathscr{G})_{0}$. Его подсемейство, состоящее из всех мер, определенных на фиксированном $\delta$-кольце $\mathscr{M}$, обозначим через $\mathfrak{R M}^{n}(T, \mathscr{G}, \mathscr{M})_{0}$. Семейство $\mathfrak{R M}^{n}(T, \mathscr{G})$, $\mathscr{B}_{c}(T, \mathscr{G})_{0}$ обозначим через $\mathfrak{R M}^{\text {no }}(T, \mathscr{G})_{0}$.

Конечную меру $\mu: \mathscr{M} \rightarrow \mathbb{R}$ на $(T, \mathscr{G})$ назовем бинарно-положительной узкой радоновской мерой, если $\mu=\mu_{1}-\mu_{2}$ для некоторых положительньх узких радоновских мер $\mu_{1}: \mathscr{M} \rightarrow \mathbb{R}_{+}$и $\mu_{2}: \mathscr{M} \rightarrow \mathbb{R}_{+}$. Если $\mu_{1}, \mu_{2} \in \mathfrak{R M}^{\text {по }}(T, \mathscr{G})_{0}$, то $\mu$ назовем бинарно-положительной узкой борелевско-радоновской мерой.

Семейство всех бинарно-положительных узких радоновских мер $\mu: \mathscr{M} \rightarrow \mathbb{R}$ на $(T, \mathscr{G})$, определенных на всех возможных $\delta$-кольцах $\mathscr{M}$, обозначим через $\mathfrak{R M}^{n}(T, \mathscr{G})$. Его подсемейство, состоящее из всех мер, определенных на фиксированном $\delta$-кольце $\mathscr{M}$, обозначим через $\mathfrak{R} \mathfrak{M}^{n}(T, \mathscr{G}, \mathscr{M})$, а подсемейство $\mathfrak{R M}^{n}\left(T, \mathscr{G}, \mathscr{B}_{c}(T, \mathscr{G})\right)$ обозначим через $\mathfrak{R M}^{n o}(T, \mathscr{G})$. Для обозначения соответствующих подсемейств ограниченных мер будем использовать нижний индекс $b$.

ПРЕДЛОЖЕНИЕ 1. Пусть $(T, \mathscr{G})$ - хаусдорфово пространство. Тогда:

1) семейство $\mathfrak{R M}^{n}(T, \mathscr{G}, \mathscr{M})\left[\mathfrak{R M}_{b}^{n}(T, \mathscr{G}, \mathscr{M})\right]$ является l-идеалом в дедекиндово полном решеточном векторном пространстве

$$
\operatorname{Meas}_{f}(T, \mathscr{M})\left[\operatorname{Meas}_{b}(T, \mathscr{M})\right]
$$

2) $\mathfrak{R M}^{n}(T, \mathscr{G}, \mathscr{M})$ является дедекиндово полным решеточным векторным пространством; наименьшие верхние (наибольиие нижние) границы подмножеств любой допустимой мощности в упорядоченных множествах $\operatorname{Meas}_{f}(T, \mathscr{M})$ и $\mathfrak{R M}^{n}(T, \mathscr{G}, \mathscr{M})$ совпадают;

3) утверждение, аналогичное 2), справедливо для $\mathfrak{R M ⿻}_{b}^{n}(T, \mathscr{G}, \mathscr{M}) \quad$ u $\operatorname{Meas}_{b}(T, \mathscr{M})$;

4) $\mathfrak{R M}_{b}^{n}(T, \mathscr{G}, \mathscr{M})$ является $l$-идеалом в $\mathfrak{R M}^{n}(T, \mathscr{G}, \mathscr{M})$;

5) $\mathfrak{R M}^{n}(T, \mathscr{G}, \mathscr{M})_{+}=\mathfrak{R M}^{n}(T, \mathscr{G}, \mathscr{M})_{0}$ u $\mathfrak{R M}_{b}^{n}(T, \mathscr{G}, \mathscr{M})_{+}=\mathfrak{R M}_{b}^{n}(T, \mathscr{G}, \mathscr{M})_{0}$.

ДоказАтЕльство. Обозначим первое семейство в 1) через $A\left[A_{b}\right]$, а второе через $B\left[B_{b}\right]$. Пусть $\mu, \nu \in A_{0}$ и $x, y \in \mathbb{R}_{+} \backslash\{0\}$. Тогда для каждого $M \in \mathscr{M}$ и каждого $\varepsilon>0$ существуют $C, D \in \mathscr{C}$ такие, что $C \cup D \subset M, \mu(M \backslash C)<\varepsilon /(2 x)$ и $\nu(M \backslash D)<\varepsilon /(2 y)$. Тогда для конечной меры $\lambda \equiv x \mu+y \nu$ на $\mathscr{M}$ мы имеем $\lambda(M \backslash C \cup D) \leqslant x \mu(M \backslash C)+y \nu(M \backslash D)<\varepsilon$. 
Кроме того, для каждой точки $t \in T$ существуют открытые окрестности $G$ и $H$ такие, что $u \equiv \sup \{\mu M \mid M \in \mathscr{M} \wedge M \subset G\}<\infty$ и $v \equiv \sup \{\nu M \mid M \in$ $\mathscr{M} \wedge M \subset H\}<\infty$. Тогда для каждого множества $M \in \mathscr{M}$ такого, что $M \subset G \cap H$, мы имеем $\lambda M \leqslant x u+y v<\infty$. Таким образом, $\lambda \in A_{0}$.

Из доказанного свойства следует, что $A=A_{0}-A_{0}$ является векторным подпространством векторного пространства $B$. Но $B$ является дедекиндово полньм решеточным векторным пространством. Пусть $\mu \in A_{0}, \nu \in B$ и $0 \leqslant \nu \leqslant \mu$. Тогда для каждых $M \in \mathscr{M}$ и $\varepsilon>0$ сушествует $C \in \mathscr{C}$ такое, что $C \subset M$ и $\mu M-\varepsilon<\mu C$. Поэтому

$$
\nu M-\nu C=\nu(M \backslash C) \leqslant \mu(M \backslash C)=\mu M-\mu C<\varepsilon
$$

означает, что $\nu M=\sup \{\nu C \mid C \in \mathscr{C} \wedge C \subset M\}$. Таким образом, $\nu$ компактно регулярно. Очевидно, что $\nu$ локально ограничено. В результате $\nu \in A_{0}$. Из доказанного свойства следует утверждение 5).

Теперь пусть $\mu \in A, \nu \in B$ и $|\nu| \leqslant|\mu|$. По определению $\mu=\mu_{1}-\mu_{2}$ для некоторых $\mu_{1}, \mu_{2} \in A_{0}$. Поэтому $|\mu| \leqslant \mu_{1}+\mu_{2} \equiv \lambda \in A_{0}$. По свойству, доказанному выше, неравенство $0 \leqslant \nu_{+} \leqslant|\nu| \leqslant \lambda$ влечет $\nu_{+} \in A_{0}$. Аналогично $-\nu_{-} \in A_{0}$. В результате $\nu=\nu_{+}-\left(-\nu_{-}\right) \in A$. Следовательно, $A$ есть $l$-идеал в $B$. Для $A_{b}$ и $B_{b}$ рассуждения аналогичны.

Остальные утверждения следуют из общей теории решеточных векторных пространств (см., например, [24], [30]).

Из утверждения 5) предложения 1 следует, что мы можем опустить слова “бинарно-положительная" и называть бинарно-положительную узкую радоновскую меру просто узкой радоновской мерой.

Таким образом, узкие радоновские меры образуют хорошее векторное пространство. Но, к сожалению, мы имеем технику лебеговского интегрирования только для широких мер. По этой причине мы вынуждены продолжать узкие радоновские меры на $\sigma$-алгебры.

Для каждой положительной узкой радоновской меры $\mu: \mathscr{M} \rightarrow \mathbb{R}_{+}$на $\delta$-кольце $\mathscr{M}$ рассмотрим ее полное сильно насышенное расширение $\mu^{\#}: \mathscr{M}^{\#} \rightarrow \overline{\mathbb{R}}_{+}$ из п. $3 \S 1$.

ПРЕДЛОЖЕНИЕ 2 . Пусть $(T, \mathscr{G})$ - хаусдорфово пространство $и \mu: \mathscr{M} \rightarrow \mathbb{R}_{+}-$ положительная узкая радоновская мера. Тогда $\mu^{\#}: \mathscr{M}^{\#} \rightarrow \overline{\mathbb{R}}_{+}$является положительной, широкой, полной и сильно насыщенной радоновской мерой, $а\left(\mu^{\#}\right)_{0}: \mathscr{B}(T, \mathscr{G}) \rightarrow \overline{\mathbb{R}}_{+}$является положительной широкой борелевско-радоновской мерой. Кроме того, если $\mu$ ограничено, то $\mu^{\#} u\left(\mu^{\#}\right)_{0}$ тоже ограничены.

ДоказАТЕЛЬСтво. Обозначим $\mathscr{M}^{\#}$ через $\mathscr{N}$ и $\mu^{\#}$ через $\nu$. Легко проверить, что $\nu$ является компактно регулярным. По определению для каждой точки $t \in T$ существует открытая окрестность $G$ такая, что $x \equiv \sup \{\mu R \mid R \in$ $\mathscr{M} \wedge R \subset G\}<\infty$. Пусть $M \in \mathscr{N}$ и $M \subset G$. Тогда для каждого компактного множества $C \subset M$ мы имеем $C \in \mathscr{M}$ и $C \subset G$; откуда $\nu C=\mu C \leqslant x$. Поэтому в силу компактной регулярности $\nu M=\sup \{\nu C \mid C \in \mathscr{C} \wedge C \subset M\} \leqslant x$. В результате $\sup \{\nu M \mid M \in \mathscr{N} \wedge M \subset G\} \leqslant x<\infty$. Таким образом, $\nu$ локально ограничено. Сходно, если $\mu$ ограничено, то $\nu$ тоже ограничено. 
Теперь надо проверить, что $\mathscr{G} \subset \mathscr{N}$. Обозначим $\mathscr{K}(T, \mathscr{M}, \mu)$ через $\mathscr{K}$ и $\stackrel{\vee}{\mu}$ через $\varkappa$. Пусть $G \in \mathscr{G}$ и $K \in \mathscr{K}$. Тогда $K=R \cup N$ для некоторых $R \in \mathscr{M}$ и $N \in \mathcal{N}(T, \mathscr{M}, \mu)$. Поэтому $G \cap K=(G \cap R) \cup(G \cap N)$. По определению узких мер $S \equiv G \cap R \in \mathscr{M}$. Кроме того, $P \equiv G \cap N \in \mathscr{N}(T, \mathscr{M}, \mu)$. В результате $G \cap K=S \cup P \in \mathscr{K}$. Это означает, что $G \in \mathscr{N}$.

Согласно п. $3 \oint 1 \nu$ является широкой, полной и сильно насышенной мерой. Согласно п. $5 \S 1 \nu_{0}$ является широкой положительной радоновской мерой. Если $\mu_{0}$ ограничено, то $\nu_{0}$ тоже ограничено.

СлЕДСТВИЕ. Пусть $\mu: \mathscr{M} \rightarrow \overline{\mathbb{R}}_{+}-$широкая положительная радоновская мера. Тогда $\mu_{00} \equiv \mu \mid \mathscr{B}_{c}(T, \mathscr{G})$ является узкой положительной борелевско-радоновской мерой и $\mu^{\#}=\left(\mu_{00}\right)^{\#}$.

Из приведенных свойств следует, что мы можем корректно определить биективные отображения

$$
\begin{aligned}
R: \mathfrak{R M}^{n o}(T, \mathscr{G})_{+} & \longmapsto \mathfrak{R M}^{w o}(T, \mathscr{G})_{0}, \\
R_{b}: \mathfrak{R M}_{b}^{n o}(T, \mathscr{G})_{+} & \longmapsto \mathfrak{R M}_{b}^{w o}(T, \mathscr{G})_{+},
\end{aligned}
$$

полагая $R \mu \equiv\left(\mu^{\#}\right)_{0}$ и $R_{b} \equiv R \mid \mathfrak{R M}_{b}^{n o}(T, \mathscr{G})_{+}$.

ПРЕДЛОЖЕНИЕ 3. Пусть $(T, \mathscr{G})$ - хаусдорфово пространство, $\mu, \nu \in$ $\mathfrak{R M}^{\text {no }}(T, \mathscr{G})_{+} u \quad x, y \in \mathbb{R}_{+} . \quad$ Тогда $R(x \mu+y \nu)=x R \mu+y R \nu . \quad$ Сходное свойство справедливо для $R_{b}$.

ДокАЗАТЕЛЬСТво. Рассмотрим положительную узкую радоновскую меру $\lambda \equiv$ $x \mu+y \nu$. По предложению из п. $5 \S 1 \pi \equiv x R \mu+y R \nu$ является положительной широкой радоновской мерой. По предложению $2 \varkappa \equiv R \lambda$ является такой же мерой. Если $C$ - компактное множество, то $\pi C=x \mu C+y \nu C=\lambda C=\varkappa C$. В силу компактной регулярности $\pi$ и $\varkappa$ мы выводим, что $\pi=\varkappa$.

Для ограниченных мер имеет место более сильное утверждение.

TЕОРема 1. Пусть $(T, \mathscr{G})$ - хаусдорфово пространство. Тогда биекиия $R_{b}$ имеет единственное расширение до изоморфизма $S_{b}: \mathfrak{R M}_{b}^{\text {no }}(T, \mathscr{G}) \longmapsto$ $\mathfrak{R M}_{b}^{w o}(T, \mathscr{G})$ между даннымм решеточными векторнылми пространствами maкого, что $S_{b} \mu=R_{b}\left(\mu_{+}\right)-R_{b}\left(-\mu_{-}\right)$.

ДокАЗАТЕльСтво. Попредложению $1 A \equiv \mathfrak{R M}_{b}^{\text {no }}(T, \mathscr{G})$-решеточное векторноепространство. По предложению из п. $5 \S 1 B \equiv \mathfrak{R M}_{b}^{w o}(T, \mathscr{G})$ - тоже решеточное векторное пространство. Поэтому по предложению 3.6.1 из [24] и предложению 3 отображение $R_{b}$ имеет единственное расширение до инъективного линейного опеpaтора $S_{b}: A \longmapsto B$ такого, что $S_{b} \mu=R_{b}\left(\mu_{+}\right)-R_{b}\left(-\mu_{-}\right)$.

Пусть $\mu, \nu \in A_{+}$. Если $\mu \leqslant \nu$, то $\left(R_{b} \mu\right) C \leqslant\left(R_{b} \nu\right) C$ для каждого компактного множества $C$. Используя компактную регулярность этих мер, мы выводим, что $R_{b} \mu M \leqslant R_{b} \nu M$ для каждого $M \in \mathscr{B}(T, \mathscr{G})$. Это означает, что $R_{b}$ является возрастающим. Если $R_{b} \mu \leqslant R_{b} \nu$, то, очевидно, $\mu \leqslant \nu$. Таким образом, $R_{b}$ является изотонным.

Следовательно, $R_{b}$ сохраняет любые границы. Поэтому $\mu \wedge \nu=0$ в $A_{+}$влечет $S_{b} \mu \wedge S_{b} \nu=R_{b} \mu \wedge R_{b} \nu=R_{b}(\mu \wedge \nu)=0$. По свойству $14 E(b)$ из [12] $S_{b}$ является решеточным линейным оператором. 
Пусть $\pi \in B$. Тогда $\pi=\pi_{+}+\pi_{-}$. По условию $\pi_{+}=R_{b} \mu^{\prime}$ и $\pi_{-}=-R_{b} \mu^{\prime \prime}$ для некоторых $\mu^{\prime}, \mu^{\prime \prime} \in A_{+}$. Рассмотрим меру $\mu=\mu^{\prime}-\mu^{\prime \prime} \in A$. Тогда $\pi=R_{b} \mu^{\prime}-$ $R_{b} \mu^{\prime \prime}=S_{b} \mu$. Таким образом, $S_{b}$ - биективный решеточный линейный оператор. Поэтому $S_{b}-$ необходимый изоморфизм.

Теперь мы докажем, что $S_{b}$ сохраняет нормы. Рассмотрим на $\mathfrak{R} \mathfrak{M}_{b}^{n o}(T, \mathscr{G})$ и $\mathfrak{R M}_{b}^{w o}(T, \mathscr{G})$ нормы, индуцированные из нормированных решеточных векторных пространств $\left(\operatorname{Meas}_{b}\left(T, \mathscr{B}_{c}(T, \mathscr{G})\right),\|\cdot\|\right)$ и $\left(\operatorname{Meas}_{b}(T, \mathscr{B}(T, \mathscr{G})),\|\cdot\|\right)$ соответственно.

ПрЕДЛОЖЕНИЕ 4. Пусть $(T, \mathscr{G})$ - хаусдорфово пространство. Тогда:

1) $\left(\mathfrak{R M}_{b}^{n o}(T, \mathscr{G}),\|\cdot\|\right)$ u $\left(\mathfrak{R M}_{b}^{w o}(T, \mathscr{G}),\|\cdot\|\right)$ являются нормированны.ми реиеточнымм векторными пространствами с аддитивнымми норммами;

2) $S_{b}:\left(\mathfrak{R M}_{b}^{n o}(T, \mathscr{G}),\|\cdot\|\right) \nrightarrow\left(\mathfrak{R M}_{b}^{w o}(T, \mathscr{G}),\|\cdot\|\right)$ является (изометрическим) изоморфизмом данных нормированных решеточных векторных пространств.

ДоКАЗАТЕЛЬСТво. 1) Обозначим первое семейство через $Y$, второе через $Z$ и $\operatorname{Meas}_{b}\left(T, \mathscr{B}_{c}(T, \mathscr{G})\right)$ через $X$. Согласно п. $1 \S 1(X,\|\cdot\|)$ является нормированным решеточным векторным пространством с аддитивной нормой $\|\mu\| \equiv|\mu|(T)$. В силу предложения $1|\mu|_{X}=|\mu|_{Y}$. Следовательно, $(Y,\|\cdot\|)$ тоже является нормированным решеточным векторным пространством с аддитивной нормой. То же самое справедливо для $(Z,\|\cdot\|)$.

2) Пусть $\mu \in Y$. Тогда по 1) $|\mu|=\mu_{+}+\left(-\mu_{-}\right)$влечет $\|\mu\|=\||\mu|\|=\left\|\mu_{+}\right\|+$ $\left\|-\mu_{-}\right\|=\mu_{+} T+\left(-\mu_{-}\right) T$. В силу компактной регулярности $\mu_{+} T=\sup \left\{\mu_{+} C \mid\right.$ $C \in \mathscr{C}\}$.

Рассмотрим меру $\pi \equiv S_{b} \mu$. По теореме $1 \pi_{+}=S_{b}\left(\mu_{+}\right)=R_{b}\left(\mu_{+}\right)$и $-\pi_{-}=S_{b}\left(-\mu_{-}\right)=R_{b}\left(-\mu_{-}\right)$. Снова в силу компактной регулярности $\left\|\pi_{+}\right\|=$ $\pi_{+} T=\sup \left\{\pi_{+} C \mid C \in \mathscr{C}\right\}=\sup \left\{\mu_{+} C \mid C \in \mathscr{C}\right\}=\mu_{+} T$. Точно так же $\left\|-\pi_{-}\right\|=\left(-\mu_{-}\right) T$. Поэтому $\|\pi\|=\left\|\pi_{+}\right\|+\left\|-\pi_{-}\right\|=\|\mu\|$. Таким образом, $S_{b}$ является изометрическим. В силу теоремы $1 S_{b}$ является необходимым изоморфизмом.

Таким образом, отображения

$$
\begin{aligned}
R: \mathfrak{R M}^{n o}(T, \mathscr{G})_{+} & \mapsto \mathfrak{R M}^{w o}(T, \mathscr{G})_{0}, \\
S_{b}: \mathfrak{R M}_{b}^{n o}(T, \mathscr{G}) & \mapsto \mathfrak{R M}_{b}^{w o}(T, \mathscr{G})
\end{aligned}
$$

дают семейство $\mathfrak{R M}^{w o}(T, \mathscr{G})_{0} \cup \mathfrak{R M}_{b}^{w o}(T, \mathscr{G})$ всех широких борелевско-радоновских мер. Но, к сожалению, это семейство не является векторным пространством. Поэтому в следующем пункте мы построим подходящее решеточное векторное пространство, содержашее семейство всех широких борелевско-радоновских мер и изоморфное решеточному векторному пространству $\mathfrak{R} \mathfrak{M}^{n o}(T, \mathscr{G})$ всех узких борелевско-радоновских мер.

2. Радоновские бимеры. Триплет $\beta \equiv\left(\mu, \mu_{1}, \mu_{2}\right)$, состоящий из мер $\mu, \mu_{1}$ и $\mu_{2}$ на $(T, \mathscr{G})$, назовем радоновским триплетом на хаусдорфовом пространстве $(T, \mathscr{G})$, если:

1) $\mu_{1}: \mathscr{M}_{1} \rightarrow \overline{\mathbb{R}}_{+}$и $\mu_{2}: \mathscr{M}_{2} \rightarrow \overline{\mathbb{R}}_{+}$являются положительными широкими радоновскими мерами; 
2) $\mu: \mathscr{M} \rightarrow \mathbb{R}$ является узкой радоновской мерой на $\delta$-кольце $\mathscr{M} \equiv \mathscr{M}_{1}^{f}\left(\mu_{1}\right) \cap$ $\mathscr{M}_{2}^{f}\left(\mu_{2}\right)$;

3) $\mu M=\mu_{1} M-\mu_{2} M$ для каждого $M \in \mathscr{M}$.

Если $\mu_{1}$ и $\mu_{2}$ принадлежат $\mathfrak{R M}^{w e}(T, \mathscr{G})_{0}$, то $\beta$ назовем расширенным радоновским триплетом. Если $\mu_{1}$ и $\mu_{2}$ принадлежат $\mathfrak{R M}^{w o}(T, \mathscr{G})_{0}$, то $\beta$ назовем борелевско-радоновским триплетом. Если $\mu_{1}$ и $\mu_{2}$ являются ограниченными, то $\beta$ назовем ограниченным.

Множество всех радоновских триплетов на $(T, \mathscr{G})$ обозначим через $R(T, \mathscr{G})$. Подмножества всех расширенных радоновских триплетов и всех борелевско-радоновских триплетов обозначим соответственно через $R^{e}(T, \mathscr{G})$ и $R^{0}(T, \mathscr{G})$. Для обозначения соответствуюших подсемейств ограниченных радоновских триплетов будем использовать нижний индекс $b$.

Радоновские триплеты $\beta \equiv\left(\mu, \mu_{1}, \mu_{2}\right)$ и $\gamma \equiv\left(\nu, \nu_{1}, \nu_{2}\right)$ назовем эквивалентными $(\beta \sim \gamma$ или $\beta \theta \gamma)$, если $\mu\left|\mathscr{B}_{c}(T, \mathscr{G})=\nu\right| \mathscr{B}_{c}(T, \mathscr{G})$. Класс эквивалентности в $R(T, \mathscr{G})$ радоновского триплета $\beta$ обозначим через $\bar{\beta} \equiv \theta \beta$. Множество всех классов $\bar{\beta}$ для всех радоновских триплетов $\beta \in R(T, \mathscr{G})$ обозначим через $\mathfrak{R} \mathfrak{B}(T, \mathscr{G})$.

Любой элемент $\mathfrak{m} \equiv \bar{\beta}$ из $\mathfrak{R B}(T, \mathscr{G})$ будем называть радоновской бимерой. Оправдание этого термина будет дано далее. Если $\beta \in R_{b}(T, \mathscr{G})$, то $\mathfrak{m} \equiv \bar{\beta}$ будем называть ограниченной радоновской бимерой. Семейство всех ограниченных радоновских бимер обозначим через $\mathfrak{R} \mathfrak{B}_{b}(T, \mathscr{G})$.

За нулевой элемент в $\mathfrak{R B}(T, \mathscr{G})$ возьмем элемент $O=\theta\left(\zeta, \zeta_{1}, \zeta_{2}\right)$, где $\zeta, \zeta_{1}$ и $\zeta_{2}$ являются нулевыми мерами на $\sigma$-алгебре $\mathscr{B}(T, \mathscr{G})$. Пусть $\beta \equiv\left(\mu, \mu_{1}, \mu_{2}\right) \sim \stackrel{\circ}{\beta} \equiv$ $\left(\stackrel{\circ}{\mu}, \stackrel{\circ}{\mu}_{1}, \stackrel{\circ}{\mu}_{2}\right)$ и $\left.\gamma \equiv\left(\nu, \nu_{1}, \nu_{2}\right) \sim \stackrel{\circ}{\gamma} \equiv(\stackrel{\circ}{\nu}, \stackrel{\circ}{\nu}, \stackrel{\circ}{\nu})_{2}\right)$ в $R(T, \mathscr{G})$. Рассмотрим $\sigma$-алгебры $\mathscr{L}_{1} \equiv \mathscr{M}_{1} \cap \mathscr{N}_{1}, \mathscr{L}_{2} \equiv \mathscr{M}_{2} \cap \mathscr{N}_{2}, \stackrel{\circ}{\mathscr{L}}_{1} \equiv \stackrel{\circ}{\mathscr{M}}_{1} \cap \stackrel{\circ}{\mathscr{N}}_{1}$ и $\stackrel{\circ}{\mathscr{L}}_{2} \equiv \stackrel{\circ}{\mathscr{M}}_{2} \cap \stackrel{\circ}{\mathscr{N}}_{2}$ и $\delta$-кольца

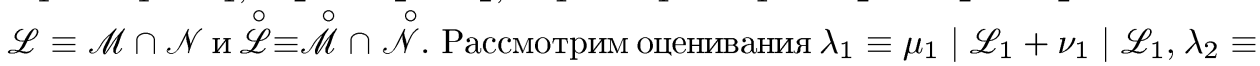
$\mu_{2}\left|\mathscr{L}_{2}+\nu_{2}\right| \mathscr{L}_{2}, \stackrel{\circ}{\lambda}_{1} \equiv \stackrel{\circ}{\mu}_{1}\left|\stackrel{\circ}{\mathscr{L}}_{1}+\stackrel{\circ}{\nu}_{1}\right| \stackrel{\circ}{\mathscr{L}}_{1}, \stackrel{\circ}{\lambda}_{2} \equiv \stackrel{\circ}{\mu}_{2}\left|\stackrel{\circ}{\mathscr{L}}_{2}+\stackrel{\circ}{\nu}_{2}\right| \stackrel{\circ}{\mathscr{L}}_{2}, \lambda \equiv \mu \mid \mathscr{L}+$ $\nu \mid \mathscr{L}$ и $\stackrel{\circ}{\lambda} \equiv \stackrel{\circ}{\mu}|\stackrel{\circ}{\mathscr{L}}+\stackrel{\circ}{\nu}| \stackrel{\circ}{\mathscr{L}}$. Рассмотрим триплеты $\alpha \equiv\left(\lambda, \lambda_{1}, \lambda_{2}\right)$ и $\stackrel{\circ}{\alpha} \equiv\left(\stackrel{\circ}{\lambda}, \stackrel{\circ}{\lambda}_{1}, \stackrel{\circ}{2}_{2}\right)$.

Лемма 1. $\alpha$ и $\stackrel{\circ}{\alpha}$ являются радоновскими триплетами, $и \alpha \sim \stackrel{\circ}{\alpha}$.

ДоКАЗАТЕЛЬСТВо. По предложению из п. $5 \S 1 \lambda_{1}$ и $\lambda_{2}$ являются положительными широкими радоновскими мерами. Следовательно, $\lambda$-мера на $\mathscr{L} \subset \mathscr{L}_{1} \cap \mathscr{L}_{2}$.

Далее,

$\mathscr{L}_{1}^{f}\left(\lambda_{1}\right)=\mathscr{L}_{1}^{f}\left(\mu_{1}\right) \cap \mathscr{L}_{1}^{f}\left(\nu_{1}\right)=\mathscr{M}_{1}^{f}\left(\mu_{1}\right) \cap \mathscr{N}_{1} \cap \mathscr{M}_{1} \cap \mathscr{N}_{1}^{f}\left(\nu_{1}\right)=\mathscr{M}_{1}^{f}\left(\mu_{1}\right) \cap \mathscr{N}_{1}^{f}\left(\nu_{1}\right)$.

Аналогично, $\mathscr{L}_{2}^{f}\left(\lambda_{2}\right)=\mathscr{M}_{2}^{f}\left(\mu_{2}\right) \cap \mathscr{N}_{2}^{f}\left(\nu_{2}\right)$. В результате

$$
\begin{gathered}
\mathscr{L}_{1}^{f}\left(\lambda_{1}\right) \cap \mathscr{L}_{2}^{f}\left(\lambda_{2}\right)=\mathscr{M}_{1}^{f}\left(\mu_{1}\right) \cap \mathscr{N}_{1}^{f}\left(\nu_{1}\right) \cap \mathscr{M}_{2}^{f}\left(\mu_{2}\right) \cap \mathscr{N}_{2}^{f}\left(\nu_{2}\right) \\
=\mathscr{M} \cap \mathscr{N}=\mathscr{L} \supset \mathscr{B}_{c}(T, \mathscr{G}) .
\end{gathered}
$$

Если $L \in \mathscr{L}$ и $G \in \mathscr{G}$, то $L \cap G \in \mathscr{M} \cap \mathscr{N}=\mathscr{L}$. В результате $\lambda_{1}^{\prime} \equiv \lambda_{1} \mid \mathscr{L}$ и $\lambda_{2}^{\prime} \equiv \lambda_{2} \mid \mathscr{L}$ являются положительными узкими радоновскими мерами. Значит, $\lambda=\lambda_{1}^{\prime}-\lambda_{2}^{\prime}$ является узкой радоновской мерой.

Наконец, если $L \in \mathscr{L}$, то $\lambda_{1} L$ и $\lambda_{2} L$ конечны и $\lambda L=\mu L+\nu L=\mu_{1} L-\mu_{2} L+\nu_{1} L-$ $\nu_{2} L=\lambda_{1} L-\lambda_{2} L$. Доказанные свойства означают, что $\alpha$ является радоновским 
триплетом. Сходно и $\stackrel{\circ}{\alpha}$ является радоновским триплетом. По условию $\mu B=\stackrel{\circ}{\mu} B$ и $\nu B=\stackrel{\circ}{\nu} B$ для каждого $B \in \mathscr{B}_{c}(T, \mathscr{G})$. Поэтому

$$
\begin{aligned}
\lambda B & =\lambda_{1} B-\lambda_{2} B=\mu_{1} B+\nu_{1} B-\mu_{2} B-\nu_{2} B=\mu B+\nu B=\stackrel{\circ}{\mu} B+\stackrel{\circ}{\nu} B \\
& =\stackrel{\circ}{\mu}_{1} B-\stackrel{\circ}{\mu}_{2} B+\stackrel{\circ}{\nu}_{1} B-\stackrel{\circ}{\nu}_{2} B=\stackrel{\circ}{\lambda}_{1} B-\stackrel{\circ}{\lambda_{2}} B=\stackrel{\circ}{\lambda} B .
\end{aligned}
$$

Таким образом, $\alpha \sim \stackrel{\circ}{\alpha}$.

Из леммы 1 следует, что мы можем корректно определить операцию сложения "+" в $\mathfrak{R B}(T, \mathscr{G})$, полагая $\bar{\beta}+\bar{\gamma} \equiv \bar{\alpha}$.

Пусть $\beta \equiv\left(\mu, \mu_{1}, \mu_{2}\right) \sim \stackrel{\circ}{\beta} \equiv\left(\stackrel{\circ}{\mu}, \stackrel{\circ}{\mu}_{1}, \stackrel{\circ}{\mu}_{2}\right)$ и $x \in \mathbb{R}$. Если $x \geqslant 0$, то рассмотрим оценивания $\pi_{1} \equiv x \mu_{1}, \pi_{2} \equiv x \mu_{2}, \stackrel{\circ}{\pi}_{1} \equiv x \stackrel{\circ}{\mu_{1}}, \stackrel{\circ}{\pi}_{2} \equiv x \stackrel{\circ}{\mu_{2}}, \pi \equiv x \mu$ и $\stackrel{\circ}{\pi} \equiv x \stackrel{\circ}{\mu}$. Если $x<0$, то рассмотрим оценивания $\varkappa_{1} \equiv-x \mu_{2}, \varkappa_{2} \equiv-x \mu_{1}, \stackrel{\circ}{\varkappa}_{1} \equiv-x \stackrel{\circ}{2}_{2}, \varkappa_{2} \equiv$ $-x \stackrel{\circ}{\mu}_{1}, \varkappa \equiv x \mu$ и $\stackrel{\circ}{\varkappa} \equiv x \stackrel{\circ}{\mu}$. Рассмотрим триплеты $\delta \equiv\left(\pi, \pi_{1}, \pi_{2}\right), \stackrel{\circ}{\delta} \equiv\left(\stackrel{\circ}{\pi}^{\circ} \stackrel{\circ}{\pi}_{1}, \stackrel{\circ}{\pi}_{2}\right)$, $\varepsilon \equiv\left(\varkappa, \varkappa_{1}, \varkappa_{2}\right)$ и $\stackrel{\circ}{\varepsilon} \equiv\left(\stackrel{\circ}{\varkappa}, \stackrel{\circ}{\varkappa_{1}}, \stackrel{\circ}{\varkappa_{2}}\right)$.

ЛЕмма 2. $\delta, \stackrel{\circ}{\delta}, \varepsilon u \stackrel{\circ}{\varepsilon}$ являются радоновскими триплетами, $\delta \sim \stackrel{\circ}{\delta} u \varepsilon \sim \stackrel{\circ}{\varepsilon}$.

ДоКАЗАТЕЛЬСТВо. По предложению из п. $5 \S 1 \varkappa_{1}$ и $\varkappa_{2}$ являются положительными широкими радоновскими мерами. По предложению 1 из п. $1 \S 2 \varkappa-$ узкая радоновская мера. Если $M \in \mathscr{M}$, то $\mu M=\mu_{1} M-\mu_{2} M$. Следовательно, $\varkappa M=\varkappa_{1} M-\varkappa_{2} M$. Это означает, что $\varepsilon-$ радоновский триплет. Сходно, $\stackrel{\circ}{\varepsilon}-$ радоновский триплет.

По условию $\mu B=\stackrel{\circ}{\mu} B$ для каждого $B \in \mathscr{B}_{c}(T, \mathscr{G})$. Поэтому $\varkappa B=\varkappa_{1} B-$ $\varkappa_{2} B=-x \mu_{2} B+x \mu_{1} B=x \mu B=x \stackrel{\circ}{\mu} B=-x \stackrel{\circ}{\mu}_{2} B+x \stackrel{\circ}{\mu}_{1} B=\stackrel{\circ}{\varkappa}_{1} B-\stackrel{\circ}{\varkappa}_{2} B=\stackrel{\circ}{\varkappa} B$. Таким образом, $\varepsilon \sim \stackrel{\circ}{\varepsilon}$.

Для $\delta$ и $\stackrel{\circ}{\delta}$ рассуждения такие же.

Из леммы 2 следует, что мы можем корректно определить композицию умножения "*” элементов из $\mathfrak{R} \mathfrak{B}(T, \mathscr{G})$ на вещественные числа, полагая $x * \bar{\beta} \equiv \bar{\delta}$ для $x \geqslant 0$ и $x * \bar{\beta} \equiv \bar{\varepsilon}$ для $x<0$.

Наша задача - доказать, что относительно операции $\bar{\beta}+\bar{\gamma}$ и композиции $x * \bar{\beta}$ семейство $\mathfrak{R B}(T, \mathscr{G})$ является векторным пространством. Для этого мы используем семейство $\mathfrak{R M}^{n o}(T, \mathscr{G})$ всех узких борелевско-радоновских мер.

Пусть $\pi \in \mathfrak{R M}^{\text {no }}(T, \mathscr{G})$. По условию $\pi=\pi_{1}-\pi_{2}$ для некоторых $\pi_{1}, \pi_{2} \in$ $\mathfrak{R M}^{n o}(T, \mathscr{G})_{+}$. Поэтому по предложению 2 из п. $1 \S 2$ меры $\mu_{1} \equiv \pi_{1}^{\#}$ и $\mu_{2} \equiv \pi_{2}^{\#}$ принадлежат семейству $\mathfrak{R} \mathfrak{M}^{w e}(T, \mathscr{G})_{0}$. Рассмотрим на $\delta$-кольце $\mathscr{M} \equiv \operatorname{dom}_{f} \mu_{1} \cap$ $\operatorname{dom}_{f} \mu_{2}$ конечную меру $\mu \equiv \mu_{1}\left|\mathscr{M}-\mu_{2}\right| \mathscr{M}$. Очевидно, что $\mu$-узкая радоновская мера. Таким образом, мы можем рассмотреть для $\pi$ триплет $\beta \equiv\left(\mu, \mu_{1}, \mu_{2}\right)$.

Сходно, если $\pi=\stackrel{\circ}{\pi}_{1}-\stackrel{\circ}{\pi}_{2}$ для некоторых $\stackrel{\circ}{\pi}_{1}, \stackrel{\circ}{\pi}_{2} \in \mathfrak{R M}^{n o}(T, \mathscr{G})_{+}$, то $\stackrel{\circ}{\mu}_{1} \equiv \stackrel{\circ \#}{\pi_{1}^{\#}}$ и $\stackrel{\circ}{\mu}_{2} \equiv \stackrel{\circ \#}{\pi_{2}^{\#}}$ принадлежат $\mathfrak{R M}^{w e}(T, \mathscr{G})_{0}$. Рассмотрим на $\delta$-кольце $\stackrel{\circ}{\mathscr{M}} \equiv \operatorname{dom}_{f} \stackrel{\circ}{\mu}_{1} \cap$ $\operatorname{dom}_{f} \stackrel{\circ}{\mu}$ узкую радоновскую меру $\stackrel{\circ}{\mu} \equiv \stackrel{\circ}{\mu}_{1}\left|\stackrel{\circ}{\mathscr{M}}-\stackrel{\circ}{\mu}_{2}\right| \stackrel{\circ}{\mathscr{M}}$. В результате мы получаем для $\pi$ другой триплет $\stackrel{\circ}{\beta} \equiv\left(\stackrel{\circ}{\mu}, \stackrel{\circ}{\mu}_{1}, \stackrel{\circ}{\mu}_{2}\right)$. 
ЛЕмма 3. $\beta$ и $\stackrel{\circ}{\beta}$ являются радоновскими триплетами, $и \beta \sim \stackrel{\circ}{\beta}$.

ДокаЗАТЕЛЬСтво. По свойству локальной конечности $\mathscr{C} \subset \mathrm{dom}_{f} \mu_{1}$. Поэтому $\mathscr{B}(T, \mathscr{G}) \subset \operatorname{dom} \mu_{1}$ влечет $\mathscr{B}_{c}(T, \mathscr{G}) \subset \operatorname{dom}_{f} \mu_{1}$, откуда $\mathscr{B}_{c}(T, \mathscr{G}) \subset \mathscr{M}$. Следовательно, $\beta$ - радоновский триплет. То же самое верно для $\stackrel{\circ}{\beta}$.

Если $B \in \mathscr{B}_{c}(T, \mathscr{G})$, то согласно определению узких радоновских мер $\mu B=$ $\mu_{1} B-\mu_{2} B=\pi_{1} B-\pi_{2} B=\pi B=\stackrel{\circ}{\pi}_{1} B-\stackrel{\circ}{\pi}_{2} B=\stackrel{\circ}{\mu}_{1} B-\stackrel{\circ}{\mu}_{2} B=\stackrel{\circ}{\mu} B$. Это означает, что $\beta \sim \stackrel{\circ}{\beta}$.

Из леммы 3 следует, что мы можем корректно определить отображение $H$ : $\mathfrak{R M}^{n o}(T, \mathscr{G}) \rightarrow \mathfrak{R B}(T, \mathscr{G})$, полагая $H \pi \equiv \bar{\beta} \equiv \overline{\bar{\circ}}$

Если $\pi \in \mathfrak{R M}_{b}^{n o}(T, \mathscr{G})$ и $\pi=\pi_{1}-\pi_{2}$, то по предложению 2 из п. $1 \S 2 \pi_{1}^{\#}$ и $\pi_{2}^{\#}$ ограничены. Поэтому $\beta \equiv\left(\mu, \pi_{1}^{\#}, \pi_{2}^{\#}\right) \in R_{b}^{e}(T, \mathscr{G})$ и $\bar{\beta} \in \mathfrak{R} \mathfrak{B}_{b}(T, \mathscr{G})$. Следовательно, мы можем корректно определить отображение $H_{b}: \mathfrak{R M}_{b}^{n o}(T, \mathscr{G}) \rightarrow \mathfrak{R} \mathfrak{B}_{b}(T, \mathscr{G})$, полагая $H_{\beta} \pi=\bar{\beta}$. Ясно, что $H_{b}=H \mid \mathfrak{R M}_{b}^{n o}(T, \mathscr{G})$.

ПРЕДЛОЖЕНИЕ 1. Пусть $(T, \mathscr{G})$ - хаусдорфово пространство. Тогда отображения $H: \mathfrak{R M}^{\text {по }}(T, \mathscr{G}) \rightarrow \mathfrak{R B}(T, \mathscr{G})$ и $H_{b}: \mathfrak{R M}_{b}^{n o}(T, \mathscr{G}) \rightarrow \mathfrak{R B}_{b}(T, \mathscr{G})$ являются биективными.

ДокАЗАТЕЛЬСтво. Пусть $\pi, \varkappa \in \mathfrak{R M}^{n o}(T, \mathscr{G})$, и предположим, что $H \pi=H \varkappa$. По определению $H \pi=\theta \beta$ для $\beta \equiv\left(\mu, \mu_{1}, \mu_{2}\right)$, где $\mu_{1} \equiv\left(\pi_{+}\right)^{\#}, \mu_{2} \equiv\left(-\pi_{-}\right)^{\#}$ и $\mu M \equiv \mu_{1} M-\mu_{2} M$ для каждого $M \in \mathscr{M} \equiv \operatorname{dom}_{f} \mu_{1} \cap \operatorname{dom}_{f} \mu_{2}$. Сходно, $H \varkappa=\theta \gamma$ для $\gamma \equiv\left(\nu, \nu_{1}, \nu_{2}\right)$, где $\nu_{1} \equiv\left(\varkappa_{+}\right)^{\#}, \nu_{2} \equiv\left(-\varkappa_{-}\right)^{\#}$ и $\nu N \equiv \nu_{1} N-\nu_{2} N$ для каждого $N \in \mathscr{N} \equiv \operatorname{dom}_{f} \nu_{1} \cap \operatorname{dom}_{f} \nu_{2}$.

Из $\beta \sim \gamma$ мы заключаем, что $\mu B=\nu B$ для каждого $B \in \mathscr{B}_{c}(T, \mathscr{G})$. В результате $\pi B=\pi_{+} B-\left(-\pi_{-}\right) B=\mu_{1} B-\mu_{2} B=\mu B=\nu B=\nu_{1} B-\nu_{2} B=\varkappa_{+} B-$ $\left(-\varkappa_{-}\right) B=\varkappa B$. Таким образом, $\pi=\varkappa$. Значит, $H$ инъективно.

Пусть $\alpha \equiv\left(\lambda, \lambda_{1}, \lambda_{2}\right) \in R(T, \mathscr{G})$, где $\lambda L=\lambda_{1} L-\lambda_{2} L$ для каждого $L \in \mathscr{L} \equiv$ $\operatorname{dom}_{f} \lambda_{1} \cap \operatorname{dom}_{f} \lambda_{2}$. Рассмотрим конечные меры $\eta \equiv \lambda, \eta_{1} \equiv \lambda_{1} \mid \mathscr{L}$ и $\eta_{2} \equiv \lambda_{2} \mid \mathscr{L}$. Очевидно, что $\eta_{1}$ и $\eta_{2}$ принадлежат $\mathfrak{R M}^{n o}(T, \mathscr{G})_{+}$. Рассмотрим меры $\mu_{1} \equiv \eta_{1}^{\#}$ и $\mu_{2} \equiv \eta_{2}^{\#}$ из $\mathfrak{R M}^{w e}(T, \mathscr{G})_{0}$. Пусть $\mathscr{M} \equiv \operatorname{dom}_{f} \mu_{1} \cap \operatorname{dom}_{f} \mu_{2}$. Рассмотрим меру $\mu \equiv \mu_{1}\left|\mathscr{M}-\mu_{2}\right| \mathscr{M}$ и триплет $\beta \equiv\left(\mu, \mu_{1}, \mu_{2}\right)$. По лемме $3 H \eta=\bar{\beta}$. Если $B \in$ $\mathscr{B}_{c}(T, \mathscr{G})$, то $\lambda_{1} B=\eta_{1} B=\mu_{1} B$ и $\lambda_{2} B=\eta_{2} B=\mu_{2} B$. По лемме 3 мы имеем $\mu B=\mu_{1} B-\mu_{2} B=\lambda_{1} B-\lambda_{2} B=\lambda B$. Это означает, что $\beta \sim \alpha$, откуда $H \eta=\bar{\alpha}$. Таким образом, $H$ сюръективно.

Если $\alpha \in R_{b}(T, \mathscr{G})$, то $\lambda_{1}$ и $\lambda_{2}$ являются ограниченными. Поэтому $\eta$ ограничено. Значит, $H_{b}$ сюръективно.

ПРЕДЛОЖЕНИЕ 2. Пусть $(T, \mathscr{G})$ - хаусдорфово пространство, $\pi, \varkappa \in$ $\mathfrak{R M}^{\text {no }}(T, \mathscr{G}) \quad u \quad x, y \in \mathbb{R}$. Тогда $H(x \pi+y \varkappa)=x * H \pi+y * H \varkappa$. Подобное свойство справедливо для $H_{b}$.

ДокАЗАТЕЛЬство. По предложению 1 из п. $1 \S 2 \rho \equiv x \pi+y \varkappa \in A \equiv$ $\mathfrak{R M}^{n o}(T, \mathscr{G})$. По определению $\pi=\pi_{1}-\pi_{2}$ и $\varkappa=\varkappa_{1}-\varkappa_{2}$ для некоторых $\pi_{i}, \varkappa_{i} \in A_{+}$. Рассмотрим меры $\mu_{1} \equiv \pi_{1}^{\#}, \mu_{2} \equiv \pi_{2}^{\#}, \nu_{1} \equiv \varkappa_{1}^{\#}$ и $\nu_{2} \equiv \varkappa_{2}^{\#}$ из $B \equiv \mathfrak{R M}^{w e}(T, \mathscr{G})_{0}$. Пусть $\mathscr{M} \equiv \operatorname{dom}_{f} \mu_{1} \cap \operatorname{dom}_{f} \mu_{2}$ и $\mathscr{N} \equiv \operatorname{dom}_{f} \nu_{1} \cap \operatorname{dom}_{f} \nu_{2}$. Рассмотрим меры $\mu \equiv \mu_{1}\left|\mathscr{M}-\mu_{2}\right| \mathscr{M}$ и $\nu \equiv \nu_{1}\left|\mathscr{N}-\nu_{2}\right| \mathscr{N}$. Тогда для триплетов $\beta \equiv\left(\mu, \mu_{1}, \mu_{2}\right)$ и $\gamma \equiv\left(\nu, \nu_{1}, \nu_{2}\right)$ мы имеем $H \pi=\bar{\beta}$ и $H \varkappa=\bar{\gamma}$. 
Предположим, что $x \geqslant 0$ и $y<0$. Тогда по определению

$$
x * \bar{\beta}=\theta\left(x \mu, x \mu_{1}, x \mu_{2}\right) \quad \text { и } y * \bar{\gamma}=\theta\left(y \nu,-y \nu_{2},-y \nu_{1}\right) .
$$

Рассмотрим $\sigma$-алгебры $\mathscr{L}_{1} \equiv \operatorname{dom} \mu_{1} \cap \operatorname{dom} \nu_{2}, \mathscr{L}_{2} \equiv \operatorname{dom} \mu_{2} \cap \operatorname{dom} \nu_{1}$ и $\delta$-кольцо $\mathscr{L} \equiv \mathscr{M} \cap \mathscr{N}$. Рассмотрим меры $\lambda_{1} \equiv x \mu_{1}\left|\mathscr{L}_{1}+\left(-y \nu_{2}\right)\right| \mathscr{L}_{1}, \lambda_{2} \equiv x \mu_{2} \mid \mathscr{L}_{2}+$ $\left(-y \nu_{1}\right) \mid \mathscr{L}_{2}$ и $\lambda \equiv x \mu|\mathscr{L}+y \nu| \mathscr{L}$. Тогда для радоновского триплета $\alpha \equiv\left(\lambda, \lambda_{1}, \lambda_{2}\right)$ мы имеем $x * \bar{\beta}+y * \bar{\gamma}=\bar{\alpha}$.

Далее рассмотрим меры $\rho_{1} \equiv x \pi_{1}-y \varkappa_{2}$ и $\rho_{2} \equiv x \pi_{2}-y \varkappa_{1}$. Тогда $\rho=\rho_{1}-\rho_{2}$, и по предложению 1 из п. $1 \S_{\circ} 2 \rho_{1}, \rho_{2} \in A_{+}$. Рассмотрим меры $\stackrel{\circ}{\lambda}_{1} \equiv \rho_{1}^{\#}$ и $\stackrel{\circ}{\lambda}_{2} \equiv \rho_{2}^{\#}$

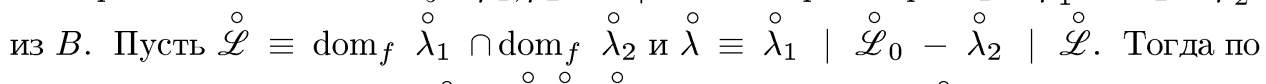
определению для триплета $\stackrel{\circ}{\alpha} \equiv\left(\stackrel{\circ}{\lambda}, \stackrel{\circ}{\lambda_{1}}, \stackrel{\circ}{\lambda_{2}}\right)$ мы имеем $H \rho=\theta \stackrel{\circ}{\alpha}$.

Если $B \in \mathscr{B}_{c}(T, \mathscr{G})$, то $\stackrel{\circ}{\lambda} B=\stackrel{\circ}{\lambda}_{1} B-\stackrel{\circ}{\lambda}_{2} B=\rho_{1} B-\rho_{2} B=x \pi_{1} B-y \varkappa_{2} B-x \pi_{2} B+$ $y \varkappa_{1} B=x \mu_{1} B-y \nu_{2} B-x \mu_{2} B+y \nu_{1} B=x \mu B+y \nu B=\lambda B$. Это означает, что $\stackrel{\circ}{\alpha} \sim \alpha$. В результате $x * H \pi+y * H \varkappa=\theta \alpha=\theta \stackrel{\circ}{\alpha}=H(x \pi+y \varkappa)$.

Для трех других возможностей для $x$ и $y$ рассуждения такие же.

СледСТВИЕ 1. Пусть $(T, \mathscr{G})$ - хаусдорфово пространство. Тогда относительно операции $\bar{\beta}+\bar{\gamma}$ и композичии $x * \bar{\beta}$ семейства $\mathfrak{R B}(T, \mathscr{G})$ и $\mathfrak{R} \mathfrak{B}_{b}(T, \mathscr{G})$ являются векторными пространствами.

ДокАЗАТЕЛЬСтво. По предложению 1 из п. $1 \S 2$ семейство $A \equiv \mathfrak{R M}^{n o}(T, \mathscr{G})$ является векторным пространством, т.е. оно удовлетворяет всем аксиомам векторного пространства. Используя свойства отображения $H$ из предложений 1 и 2 этого пункта, мы можем вывести, что семейство $B \equiv \mathfrak{R} \mathfrak{B}(T, \mathscr{G})$ удовлетворяет также всем необходимым аксиомам.

Проверим, например, что $x *(y * b)=(x y) * b$. По предложению $1 b=H a$ для некоторого $a \in A$. Из $x(y a)=(x y) a$ мы заключаем по предложению 2 , что $x *(y * b)=x *(y * H a)=x * H(y a)=H(x(y a))=H((x y) a)=(x y) * H a=(x y) * b$. Для ограниченного случая рассуждения такие же.

СлЕДСТВИЕ 2. Пусть $(T, \mathscr{G})$ - хаусдорфово пространство. Тогда $H$ : $\mathfrak{R M}^{n o}(T, \mathscr{G}) \longmapsto \mathfrak{R B}(T, \mathscr{G})$ и $H_{b}: \mathfrak{R M}_{b}^{n o}(T, \mathscr{G}) \longmapsto \mathfrak{R B}_{b}(T, \mathscr{G})$ являются изоморфизмами данных векторных пространств.

ДокАЗАТЕЛЬСтво. В силу предложения 2 и следствия 1 п. $2 \S 2$ H является гомоморфизмом данных векторных пространств. В силу предложения 1 п. $2 \S 2 \mathrm{H}$ есть изоморфизм. Для $H_{b}$ рассуждения такие же.

Далее вместо $x * \bar{\beta}$ мы будем писать просто $x \bar{\beta}$.

Теперь определим порядок на векторном пространстве $\mathfrak{R} \mathfrak{B}(T, \mathscr{G})$.

ЛЕмма 4. Пусть $\beta \equiv\left(\mu, \mu_{1}, \mu_{2}\right), \stackrel{\circ}{\beta} \equiv\left(\stackrel{\circ}{\mu}, \stackrel{\circ}{\mu_{1}}, \stackrel{\circ}{\mu}_{2}\right), \quad \gamma \equiv\left(\nu, \nu_{1}, \nu_{2}\right), \quad u \quad \stackrel{\circ}{\gamma} \equiv$ $\left(\stackrel{\circ}{\nu}, \stackrel{\circ}{\nu}, \stackrel{\circ}{\nu}_{2}\right)$ - радоновские триплеты такие, что $\beta \sim \stackrel{\circ}{\beta}$ u $\gamma \sim \stackrel{\circ}{\gamma}$. Тогда следующие утверждения равносильны:

1) $\mu B \leqslant \nu B$ для каждого $B \in \mathscr{B}_{c}(T, \mathscr{G})$;

2) $\stackrel{\circ}{\mu} B \leqslant \stackrel{\circ}{\nu} B$ для каждого $B \in \mathscr{B}_{c}(T, \mathscr{G})$. 
ДокаЗАтЕЛЬство. Утверждение следует из равенств $\mu B=\stackrel{\circ}{\mu} B$ и $\nu B=\stackrel{\circ}{\nu} B$ для каждого $B \in \mathscr{B}_{c}(T, \mathscr{G})$.

Из леммы 4 следует, что мы можем корректно определить бинарное отношение $\omega$ на $\mathfrak{R B}(T, \mathscr{G})$, полагая $\bar{\beta} w \bar{\gamma}$, если $\stackrel{\circ}{\mu} B \leqslant \stackrel{\circ}{\nu} B$ для каждого $B \in \mathscr{B}_{c}(T, \mathscr{G})$ и некоторых $\stackrel{\circ}{\beta} \equiv\left(\stackrel{\circ}{\mu}, \stackrel{\circ}{\mu}_{1}, \stackrel{\circ}{\mu}_{2}\right) \in \bar{\beta}$ и $\stackrel{\circ}{\gamma} \equiv\left(\stackrel{\circ}{\nu}, \stackrel{\circ}{\nu}_{1}, \stackrel{\circ}{\nu}_{2}\right) \in \bar{\gamma}$.

ЛЕмма 5. Отношение $\omega$ является отношением порядка.

ДокаЗАтельство. Очевидно, что $\bar{\beta} \omega \bar{\beta}$. Пусть $\bar{\beta} \omega \bar{\gamma}$ и $\bar{\gamma} \omega \bar{\beta}$. Тогда $\mu B \leqslant \nu B$ и $\stackrel{\circ}{\nu} B \leqslant \stackrel{\circ}{\mu} B$ для каждого $B \in \mathscr{B}_{c}(T, \mathscr{G})$ и некоторых $\beta \equiv\left(\mu, \mu_{1}, \mu_{2}\right) \in \bar{\beta}, \stackrel{\circ}{\beta} \equiv$ $\left(\stackrel{\circ}{\mu}, \stackrel{\circ}{\mu}_{1}, \stackrel{\circ}{\mu}_{2}\right) \in \bar{\beta}, \gamma \equiv\left(\nu, \nu_{1}, \nu_{2}\right) \in \bar{\gamma}$ и $\stackrel{\circ}{\gamma} \equiv\left(\stackrel{\circ}{\nu}, \stackrel{\circ}{\nu}_{1}, \stackrel{\circ}{\nu}_{2}\right) \in \bar{\gamma}$. Из $\mu B=\stackrel{\circ}{\mu} B$ и $\nu B=\stackrel{\circ}{\nu} B$ мы заключаем, что $\nu B=\stackrel{\circ}{\nu} B \leqslant \stackrel{\circ}{\mu} B=\mu B$, откуда $\nu B=\mu B$ для каждого $B$. Значит, $\gamma \sim \beta$ и $\bar{\gamma}=\bar{\beta}$.

Наконец, пусть $\bar{\beta} \omega \bar{\gamma}$ и $\bar{\gamma} \omega \bar{\delta}$. Тогда $\mu B \leqslant \nu B$ и $\stackrel{\circ}{\nu} B \leqslant \stackrel{\circ}{\pi} B$ для каждого $B \in$ $\mathscr{B}_{c}(T, \mathscr{G})$ и некоторых $\beta \equiv\left(\mu, \mu_{1}, \mu_{2}\right) \in \bar{\beta}, \gamma \equiv\left(\nu, \nu_{1}, \nu_{2}\right) \in \bar{\gamma}, \stackrel{\circ}{\gamma} \equiv\left(\stackrel{\circ}{\nu}, \stackrel{\circ}{\nu}_{1}, \stackrel{\circ}{\nu_{2}}\right) \in \bar{\gamma}$ и $\stackrel{\circ}{\delta} \equiv\left(\stackrel{\circ}{\pi}, \stackrel{\circ}{\pi}_{1}, \stackrel{\circ}{\pi}_{2}\right) \in \bar{\delta}$. Из $\nu B=\stackrel{\circ}{\nu} B$ мы заключаем, что $\mu B \leqslant \nu B=\stackrel{\circ}{\nu} B \leqslant \stackrel{\circ}{\pi} B$. Значит, по определению $\omega$ мы получаем $\bar{\beta} \omega \bar{\delta}$.

Теорема 1. Пусть $(T, \mathscr{G})$ - хаусдорфово пространство. Тогда:

1) отображсеня $H \quad$ и $H_{\beta}$ являются изотонными;

2) семейства $\mathfrak{R B}(T, \mathscr{G}) \quad$ и $\mathfrak{R B}_{b}(T, \mathscr{G})$ являются дедекиндово полными решеточно упорядоченными векторными пространствами и второе из них является $l$-идеалом в первом;

3) отображения $H: \mathfrak{R M}^{\text {no }}(T, \mathscr{G}) \longmapsto \mathfrak{R B}(T, \mathscr{G}) \quad u \quad H_{b}: \mathfrak{R M}_{b}^{\text {no }}(T, \mathscr{G}) \longmapsto$ $\mathfrak{R B}_{b}(T, \mathscr{G})$ являются изоморфизмами данных решеточных векторных пространств.

ДоказАТЕЛЬство. 1) Пусть $\pi, \varkappa \in A \equiv \mathfrak{R M}^{\text {no }}(T, \mathscr{G})$. По определению $\pi=$ $\pi_{1}-\pi_{2}$ и $\varkappa=\varkappa_{1}-\varkappa_{2}$ для некоторых положительных борелевско-радоновских мер. Рассмотрим $\mu_{i} \equiv \pi_{i}^{\#}, \nu_{i} \equiv \varkappa_{i}^{\#}, \mathscr{M}_{i} \equiv \operatorname{dom} \mu_{i}, N_{i} \equiv \operatorname{dom} \nu_{i}, \mathscr{M} \equiv \mathscr{M}_{1}^{f}\left(\mu_{1}\right) \cap$ $\mathscr{M}_{2}^{f}\left(\mu_{2}\right), \mathscr{N} \equiv \mathscr{N}_{1}^{f}\left(\nu_{1}\right) \cap \mathscr{N}_{2}^{f}\left(\nu_{2}\right), \mu \equiv \mu_{1}\left|\mathscr{M}-\mu_{2}\right| \mathscr{M}$ и $\nu \equiv \nu_{1}\left|\mathscr{N}-\nu_{2}\right| \mathscr{N}$. Тогда по определению $H$ для триплетов $\beta \equiv\left(\mu, \mu_{1}, \mu_{2}\right)$ и $\gamma \equiv\left(\nu, \nu_{1}, \nu_{2}\right)$ мы имеем $H \pi=\bar{\beta}$ и $Н \varkappa=\bar{\gamma}$.

Если $\pi \leqslant \varkappa$, то $B \in \mathscr{B}_{c}(T, \mathscr{G})$ влечет $\mu B=\mu_{1} B-\mu_{2} B=\pi_{1} B-\pi_{2} B \leqslant \varkappa_{1} B-$ $\varkappa_{2} B=\nu_{1} B-\nu_{2} B=\nu B$. Это дает $H \pi=\bar{\beta} \leqslant \bar{\gamma}=H \varkappa$.

Теперь пусть $\bar{\beta}=H \pi \leqslant H \varkappa=\bar{\gamma}$. Тогда по лемме $5 \mu B \leqslant \nu B$ для каждого $B$. Поэтому $\pi B=\pi_{1} B-\pi_{2} B=\mu_{1} B-\mu_{2} B=\mu B \leqslant \nu B=\nu_{1} B-\nu_{2} B=\varkappa_{1} B-$ $\varkappa_{2} B=\varkappa B$, т.е. $\pi \leqslant \varkappa$.

2) Из 1) следует, что отображение $H^{-1}$ тоже изотонно. Следовательно, $H$ и $H^{-1}$ сохраняют любые границы. Кроме того, по следствию 2 из предложения 2 этого пункта $H$ является изоморфизмом данных векторных пространств. Пусть $E \subset B \equiv \mathfrak{R B}(T, \mathscr{G})$ и $b \in B$ является верхней гранищей для $E$. Рассмотрим множество $D \equiv H^{-1}[E]$ и элемент $a \equiv H^{-1} b$. Если $d \in D$, то $H d \leqslant b=H a$ влечет $d \leqslant a$. Поэтому по предложению 1 из п. $1 \S 2$ существует $d_{0} \equiv \sup D$. В результате $H d_{0}=\sup H[D]=\sup E$. 
Если $E$ ограничено снизу, то рассуждения такие же. Таким образом, $B$ дедекиндово полно.

Пусть $b_{1}, b_{2} \in B$. Рассмотрим элементы $a_{i} \equiv H^{-1} b_{i}, a^{\prime} \equiv a_{1} \vee a_{2}$ и $a^{\prime \prime} \equiv a_{1} \wedge a_{2}$. Тогда $H a^{\prime}=H a_{1} \vee H a_{2}=b_{1} \vee b_{2}$ и $H a^{\prime \prime}=H a_{1} \wedge H a_{2}=b_{1} \wedge b_{2}$. Следовательно, упорядоченное множество $B$ является решеточно упорядоченным.

Сходным образом проверяется, что $B$ является решеточным векторным пространством. Например, пусть $b, b_{1}, b_{2} \in B$ и $b_{1} \leqslant b_{2}$. Возьмем $a_{i} \equiv H^{-1} b_{i}$ и $a \equiv H^{-1} b$. Тогда $a_{1} \leqslant a_{2}$ влечет $a_{1}+a \leqslant a_{2}+a$. Применяя $H$, мы получаем

$$
b_{1}+b=H\left(a_{1}+a\right) \leqslant H\left(a_{2}+a\right)=b_{2}+b .
$$

3) Из $H\left(a_{1} \vee a_{2}\right)=H a_{1} \vee H a_{2}$ и $H\left(a_{1} \wedge a_{2}\right)=H a_{1} \wedge H a_{2}$ и следствия 2 предложения 2 этого пункта следует, что $H: A \rightarrow B$ является гогоморфизмом данных решеточных векторных пространств. Так как $H$ биективно по предложению 1 , мы заключаем, что $H$ является необходимым изоморфизмом.

Для семейств $A_{b} \equiv \mathfrak{R M}_{b}^{n o}(T, \mathscr{G})$ и $B_{b} \equiv \mathfrak{R} \mathfrak{B}_{b}(T, \mathscr{G})$ и для отображения $H_{b}$ рассуждения такие же. Нам нужно только проверить, что $B_{b}$ есть $l$-идеал в $B$.

Пусть $b \in B_{b}, d \in B$ и $|d| \leqslant|b|$. Возьмем $a \equiv H^{-1} b \in A$ и $c \equiv H^{-1} d \in A$. Так как $H_{b}$ есть ограничение $H$, имеем $a \in A_{b}$. Так как $H$ есть изоморфизм, вьводим, что $|c| \leqslant|a|$ в $A$. Теперь по предложению 1 из п. $1 c \in A_{b}$. В результате $d=H_{b} c \in B_{b}$.

Теперь мы свяжем положительные широкие радоновские меры и радоновские бимеры. Конусы положительных элементов в решеточных векторных пространствах $\mathfrak{R} \mathfrak{B}(T, \mathscr{G})$ и $\mathfrak{R} \mathfrak{B}_{b}(T, \mathscr{G})$ будут обозначаться, как обычно, через $\mathfrak{R} \mathfrak{B}(T, \mathscr{G})_{+}$и $\mathfrak{R B}_{b}(T, \mathscr{G})_{+}$соответственно.

Для каждой меры $\mu \in \mathfrak{R M}^{w o}(T, \mathscr{G})_{0}$ возьмем узкую положительную радоновскую меру $\mu_{f} \equiv \mu \mid \operatorname{dom}_{f} \mu$, радоновский триплет $\beta \equiv\left(\mu_{f}, \mu, 0\right)$ и положительную радоновскую бимеру $\mathfrak{m} \equiv \bar{\beta}$. Эта процедура определяет отображение $E: \mathfrak{R M}^{w o}(T, \mathscr{G})_{0} \rightarrow \mathfrak{R B}(T, \mathscr{G})_{+}$такое, что $E \mu \equiv \mathfrak{m}$. Если $\mu \in$ $\mathfrak{R M}_{b}^{w o}(T, \mathscr{G})_{+}$, то $E \mu \in \mathfrak{R B}_{b}(T, \mathscr{G})_{+}$. Поэтому мы можем определить отображение $E_{b}: \mathfrak{R M}_{b}^{w o}(T, \mathscr{G})_{+} \rightarrow \mathfrak{R B}_{b}(T, \mathscr{G})_{+}$, полагая $E_{b} \equiv E \mid \mathfrak{R M}_{b}^{w o}(T, \mathscr{G})_{+} \cdot$

Теперь мы можем доказать основной результат этого пункта.

Теорема 2. Пусть $(T, \mathscr{G})$ - хаусдорфово пространство. Тогда:

1) отображения $E: \mathfrak{R M}^{w o}(T, \mathscr{G})_{0} \rightarrow \mathfrak{R B}(T, \mathscr{G})_{+} \quad u E_{b}: \mathfrak{R M}_{b}^{w o}(T, \mathscr{G})_{+} \rightarrow$ $\mathfrak{R B}_{b}(T, \mathscr{G})_{+}$являются биективными;

2) ecлu $\mu, \nu \in \mathfrak{R M}^{w o}(T, \mathscr{G})_{0} \quad u \quad x, y \in \mathbb{R}_{+}$, mo $E(x \mu+y \nu)=x E \mu+y E \nu$; сходное свойство справедливо для $E_{b}$;

3) отображсния $E \quad$ u $E_{b}$ являются изотонными и сохраняют любые порядковие гранищы;

4) для каждой бимеры $\mathfrak{m} \in \mathfrak{R} \mathfrak{B}(T, \mathscr{G})$ существуют единственные меры $\mu^{\prime}, \mu^{\prime \prime} \in \mathfrak{R M}^{w o}(T, \mathscr{G})_{0}$ такие, что $\mathfrak{m}=E \mu^{\prime}-E \mu^{\prime \prime}, E \mu^{\prime}=\mathfrak{m}_{+} \quad u \quad E \mu^{\prime \prime}=-\mathfrak{m}_{-}$; если м⿰ ограничено, то $\mu^{\prime}$ и $\mu^{\prime \prime}$ тожсе ограничены. 
ДокаЗАТЕЛЬСтво. Пусть $E \mu=E \nu$. Это означает, что $\mu_{f} B=\nu_{f} B$ для каждого $B \in \mathscr{B}_{c}(T, \mathscr{G})$. В силу компактной регулярности этих мер $\mu=\nu$. Таким образом, $E$ инъективно.

Рассмотрим меру $\pi \equiv x \mu+y \nu \in \mathfrak{R M}^{w o}(T, \mathscr{G})_{0}$. Предположим, что $x>0$ и $y>0$. По определению $E \mu=\bar{\beta}, E \nu=\bar{\gamma}$ и $E \pi=\bar{\delta}$ для $\beta \equiv\left(\mu_{f}, \mu, 0\right), \gamma \equiv\left(\nu_{f}, \nu, 0\right)$ и $\delta=\left(\pi_{f}, \pi, 0\right)$. Ясно, что $\pi_{f}=x \mu_{f}+y \nu_{f}$. Рассмотрим $\sigma$-алгебры $\mathscr{L}_{1} \equiv \mathscr{L}_{2} \equiv$ $\mathscr{B}(T, \mathscr{G})$ и $\delta$-кольцо $\mathscr{L} \equiv \operatorname{dom}_{f}(x \mu) \cap \operatorname{dom}_{f}(y \nu)=\operatorname{dom}_{f} \nu \cap \operatorname{dom}_{f} \nu=\operatorname{dom}_{f} \pi$. Рассмотрим меры $\lambda_{1} \equiv \pi$ на $\mathscr{L}_{1}, \lambda_{2} \equiv 0$ на $\mathscr{L}_{2}, \lambda \equiv \pi_{f}$ на $\mathscr{L}$ и соответствуюший радоновский триплет $\alpha \equiv\left(\lambda, \lambda_{1}, \lambda_{2}\right)$. По определению $x \bar{\beta}=\theta\left(x \mu_{f}, x \mu, 0\right), y \bar{\gamma}=$ $\theta\left(y \nu_{f}, y \nu, 0\right)$ и $x E \mu+y E \nu=x \bar{\beta}+y \bar{\gamma}=\bar{\alpha}=\theta\left(\pi_{f}, \pi, 0\right)=E \pi$.

Пусть $\mathfrak{m} \in \mathfrak{R B}(T, \mathscr{G})$ и $\mathfrak{m}=\bar{\beta}$ для некоторого $\beta \equiv\left(\mu, \mu_{1}, \mu_{2}\right) \in R(T, \mathscr{G})$. Рассмотрим широкие борелевско-радоновские меры $\nu^{\prime} \equiv \mu_{1} \mid \mathscr{B}(T, \mathscr{G})$ и $\nu^{\prime \prime} \equiv$ $\mu_{2} \mid \mathscr{B}(T, \mathscr{G})$. Рассмотрим $\delta$-кольцо $\mathscr{N} \equiv \operatorname{dom}_{f} \nu^{\prime} \cap \operatorname{dom}_{f} \nu^{\prime \prime} \subset \operatorname{dom}_{f} \mu$ и конечную меру $\nu \equiv \nu^{\prime}\left|\mathscr{N}-\nu^{\prime \prime}\right| \mathscr{N}$. Тогда мы получаем радоновский триплет $\gamma \equiv\left(\nu, \nu^{\prime}, \nu^{\prime \prime}\right)$. Из $\nu B=\nu^{\prime} B-\nu^{\prime \prime} B=\mu_{1} B-\mu_{2} B=\mu B$ для каждого $B \in \mathscr{B}_{c}(T, \mathscr{G})$ заключаем, что $\beta \sim \gamma$. Таким образом, $\mathfrak{m}=\bar{\gamma}$. По определению отображения $E$ имеем $E \nu^{\prime}=\theta\left(\nu_{f}^{\prime}, \nu^{\prime}, 0\right)$ и $E \nu^{\prime \prime}=\theta\left(\nu_{f}^{\prime \prime}, \nu^{\prime \prime}, 0\right)$. Соответственно по определению композиции имеем $-E \nu^{\prime \prime}=\theta\left(-\nu_{f}^{\prime \prime}, 0, \nu^{\prime \prime}\right)$. Рассмотрим $\sigma$-алгебры $\mathscr{L}_{1} \equiv \mathscr{L}_{2}=$ $\mathscr{B}(T, \mathscr{G})$ и меры $\lambda_{1} \equiv \nu^{\prime}$ на $\mathscr{L}_{1}$ и $\lambda_{2} \equiv \nu^{\prime \prime}$ на $\mathscr{L}_{2}$. Рассмотрим $\delta$-кольцо $\mathscr{L} \equiv$ $\operatorname{dom}_{f}\left(\nu_{f}^{\prime}\right) \cap \operatorname{dom}_{f}\left(-\nu_{f}^{\prime \prime}\right)=\mathscr{N}$, мepу $\lambda \equiv \nu_{f}^{\prime}\left|\mathscr{L}+\left(-\nu_{f}^{\prime \prime}\right)\right| \mathscr{L}=\nu^{\prime}\left|\mathscr{N}-\nu^{\prime \prime}\right| \mathscr{N}=\nu$ и радоновский триплет $\alpha \equiv\left(\lambda, \lambda_{1}, \lambda_{2}\right)$. По определению сложения мы имеем $E \nu^{\prime}-$ $E \nu^{\prime \prime}=\bar{\alpha}=\theta\left(\nu, \nu^{\prime}, \nu^{\prime \prime}\right)=\mathfrak{m}$. Если бимера $\mathfrak{m}$ ограничена, то $\beta \in R_{b}(T, \mathscr{G})$ и, следовательно, $\nu^{\prime}$ и $\nu^{\prime \prime}$ ограничены.

Теперь предположим, что $\mathfrak{m}=\bar{\gamma} \in \mathfrak{R B}(T, \mathscr{G})_{+}$, т.е. $\bar{\gamma} \geqslant \theta\left(\zeta, \zeta_{1}, \zeta_{2}\right)$. По определению порядка в $\mathfrak{R B}(T, \mathscr{G})_{+}$мы имеем $\nu B \geqslant \zeta B=0$ для каждого $B \in \mathscr{B}_{c}(T, \mathscr{G})$. По следствию к предложению 2 из п. $1 \S 2 \nu_{00}^{\prime}, \nu_{00}^{\prime \prime} \in \mathfrak{R M}^{n o}(T, \mathscr{G})_{+}$. По определению равенство $\nu_{00}=\nu_{00}^{\prime}-\nu_{00}^{\prime \prime} \geqslant 0$ означает, что $\nu_{00} \in \mathfrak{R M}^{n o}(T, \mathscr{G})_{+}$. Рассмотрим меру $\pi \equiv\left(\nu_{00}\right)^{\#} \in \mathfrak{R M}^{w e}(T, \mathscr{G})_{0}$ из предложения 2 п. $1 \S 2$. Если $\mathfrak{m}$ ограничено, то $\pi$ тоже ограничено. Наконец, рассмотрим меру $\lambda \equiv \pi \mid \mathscr{B}(T, \mathscr{G}) \in$ $\mathfrak{R M}^{w o}(T, \mathscr{G})_{0}$.

Для $\lambda$ возьмем соответствуюший радоновский триплет $\alpha \equiv\left(\lambda_{f}, \lambda, 0\right)$. Если $B \in \mathscr{B}_{c}(T, \mathscr{G})$, то $\lambda_{f} B=\lambda B=\pi B=\nu_{00} B=\nu B$. Это означает, что $\alpha \sim \gamma$. В результате $\mathfrak{m}=\bar{\gamma}=\bar{\alpha}=E \lambda$. Таким образом, $E$ сюръективно и, значит, биективно. Если $\mathfrak{m}$ ограничено, то $\lambda$ и $\alpha$ тоже ограничены. Следовательно, $E_{b}$ биективно.

Пусть $\mu \leqslant \nu$ в $\mathfrak{R M}^{w o}(T, \mathscr{G})_{0}$. Тогда $\mu_{f} B \leqslant \nu_{f} B$ для каждого $B \in \mathscr{B}_{c}(T, \mathscr{G})$ означает, что $E \mu=\theta\left(\mu_{f}, \mu, 0\right) \leqslant \theta\left(\nu_{f}, \nu, 0\right)=E \nu$. Обратно, пусть $E \mu \leqslant E \nu$ в $\mathfrak{R} \mathfrak{B}(T, \mathscr{G})_{+}$. По определению $\stackrel{\circ}{\mu} B \leqslant \stackrel{\circ}{\nu} B$ для каждого $B$ и некоторых $\stackrel{\circ}{\beta} \equiv$ $\left.\left(\stackrel{\circ}{\mu}, \stackrel{\circ}{\mu}_{1}, \stackrel{\circ}{\mu}_{2}\right) \in E \mu, \stackrel{\circ}{\gamma} \equiv\left(\stackrel{\circ}{\nu}, \stackrel{\circ}{\nu}_{1}, \stackrel{\circ}{\nu}\right)_{2}\right) \in E \nu$. Согласно лемме 4 этого пункта $\mu_{f} B \leqslant \nu_{f} B$. Поэтому для каждого компактного множества $\mathscr{C}$ имеем $\mu \mathscr{C} \leqslant \nu \mathscr{C}$. Если $M \in \mathscr{B}(T, \mathscr{G})$, то в силу компактной регулярности этих мер получаем

$$
\mu M=\sup \{\mu C \mid C \in \mathscr{C} \wedge C \subset M\} \leqslant \sup \{\nu C \mid C \in \mathscr{C} \wedge C \subset M\}=\nu M .
$$

Поэтому $\mu \leqslant \nu$. Таким образом, $E$ является изотонным. Следовательно, $E$ сохраняет любые порядковые границы. То же самое справедливо для $E_{b}$. 
Пусть $\mathfrak{m} \in \mathfrak{R} \mathfrak{B}(T, \mathscr{G})$. Тогда в силу теоремы 1 этого пункта $\mathfrak{m}=\mathfrak{m}_{+}+\mathfrak{m}_{-}$. Так как $E$ биективно, $\mathfrak{m}_{+}=E \mu^{\prime}$ и $-\mathfrak{m}_{-}=E \mu^{\prime \prime}$ для единственных мер $\mu^{\prime}$ и $\mu^{\prime \prime}$. Если m ограничено, то по 1) $\mu^{\prime}$ и $\mu^{\prime \prime}$ тоже ограничены.

Отображение $E: \mathfrak{R M} \mathfrak{M o}^{\text {wo }}(T, \mathscr{G})_{0} \longmapsto \mathfrak{R B}(T, \mathscr{G})_{+}$назовем каноническим вложением семейства всех (иироких) положительных борелевско-радоновских мер в решеточное векторное пространство всех радоновских бимер. Меры $\mu^{\prime}$ и $\mu^{\prime \prime}$ назовем родительскими положительньми (широкими) борелевско-радоновскими мерами радоновской бимеры $\mathfrak{m}$ и обозначим через $p^{\prime} \mathfrak{m}$ и $p^{\prime \prime} \mathfrak{m}$ соответственно.

Между бимерой $\mathfrak{m}$ и ее родительскими мерами $p^{\prime} \mathfrak{m}$ и $p^{\prime \prime} \mathfrak{m}$ имеется следующая связь.

ПРЕДЛОЖЕНИЕ 3. Пусть $(T, \mathscr{G})$ - хаусдорфово пространство, и пусть $\mathfrak{m} \in$ $\mathfrak{R B}(T, \mathscr{G})$. Тогда $\left(\mu, p^{\prime} \mathfrak{m}, p^{\prime \prime} \mathfrak{m}\right) \in R(T, \mathscr{G}) u \mathfrak{m}=\theta\left(\mu, p^{\prime} \mathfrak{m}, p^{\prime \prime} \mathfrak{m}\right)$, где $\mu \equiv p^{\prime} \mathfrak{m} \mid \mathscr{M}-$ $p^{\prime \prime} \mathfrak{m} \mid \mathscr{M}$ есть узкая радоновская мера на $\delta$-кольче $\mathscr{M} \equiv \operatorname{dom}_{f}\left(p^{\prime} \mathfrak{m}\right) \cap \operatorname{dom}_{f}\left(p^{\prime \prime} \mathfrak{m}\right)$.

ДокАЗАТЕЛЬство. Обозначим $p^{\prime} \mathfrak{m}$ и $p^{\prime \prime} \mathfrak{m}$ через $\mu^{\prime}$ и $\mu^{\prime \prime}$ соответственно. Очевидно, что $\mu_{1} \equiv \mu^{\prime} \mid \mathscr{M}$ и $\mu_{2} \equiv \mu^{\prime \prime} \mid \mathscr{M}$ являются положительными узкими радоновскими мерами. Поэтому по определению $\mu=\mu_{1}-\mu_{2}$ есть узкая радоновская мера. Ясно также, что $\left(\mu, \mu^{\prime}, \mu^{\prime \prime}\right)$ является радоновским триплетом. По определению отображения $E$ мы имеем $E \mu^{\prime}=\theta\left(\mu_{f}^{\prime}, \mu^{\prime}, 0\right)$ и $E \mu^{\prime \prime}=\theta\left(\mu_{f}^{\prime \prime}, \mu^{\prime \prime}, 0\right)$. По определению композиции имеем $-E \mu^{\prime \prime}=\theta\left(-\mu_{f}^{\prime \prime}, 0, \mu^{\prime \prime}\right)$. Из определения сложения выводим, как и в доказательстве теоремы 2 этого пункта, что $\mathfrak{m}=E \mu^{\prime}-E \mu^{\prime \prime}=\theta\left(\mu, \mu^{\prime}, \mu^{\prime \prime}\right)$.

ПРЕДЛОЖЕНИЕ 4. Пусть $(T, \mathscr{G})$ - хаусдорфово пространство, и пусть $\mathfrak{m} \in$ $\mathfrak{R B}(T, \mathscr{G}) . \quad$ Ecлu $\gamma \equiv\left(\nu, \nu_{1}, \nu_{2}\right) \in \mathfrak{m} \quad u \quad \mu \equiv \nu \mid \mathscr{B}_{c}(T, \mathscr{G})$, mo $p^{\prime} \mathfrak{m}=\left(\left(\mu_{+}\right)^{\#}\right)_{0} u$ $p^{\prime \prime} \mathfrak{m}=\left(\left(-\mu_{-}\right)^{\#}\right)_{0}$.

ДоказАТельство. Обозначим $p^{\prime} \mathfrak{m}$ и $p^{\prime \prime} \mathfrak{m}$ через $\mu^{\prime}$ и $\mu^{\prime \prime}$ соответственно. В силу предложения 1 из п. $1 \S 2 \mu=\mu_{+}+\mu_{-}$. А в силу теорем 1 и 2 этого пункта $E \mu^{\prime}=H\left(\mu_{+}\right)$и $E \mu^{\prime \prime}=H\left(-\mu_{-}\right)$.

Для $\mu_{+}$возьмем $\varkappa_{1} \equiv \mu_{+}$и $\varkappa_{2} \equiv 0$. Тогда $\varkappa_{1}^{\#}=\left(\mu_{+}\right)^{\#}$ и $\varkappa_{2}^{\#}=0$. Мы можем предположить, что $\operatorname{dom} \varkappa_{2}^{\#}=\operatorname{dom} \varkappa_{1}^{\#}=\operatorname{dom}\left(\mu_{+}\right)^{\#}$. Рассмотрим на $\delta$-кольце $\mathscr{K} \equiv \operatorname{dom}_{f}\left(\mu_{+}\right)^{\#}$ положительную узкую радоновскую меру $\varkappa \equiv \varkappa_{1}^{\#} \mid \mathscr{K}=\left(\mu_{+}\right)^{\#}$. Тогда по определению $H$ мы имеем $H\left(\mu_{+}\right)=\theta\left(\left(\mu_{+}\right)_{f}^{\#},\left(\mu_{+}\right)^{\#}, 0\right)$.

Аналогично, $H\left(-\mu_{-}\right)=\theta\left(\left(-\mu_{-}\right)_{f}^{\#},\left(-\mu_{-}\right)^{\#}, 0\right)$.

По определению $E$ имеем $E \mu^{\prime}=\theta\left(\mu_{f}^{\prime}, \mu^{\prime}, 0\right)$ и $E \mu^{\prime \prime}=\theta\left(\mu_{f}^{\prime \prime}, \mu^{\prime \prime}, 0\right)$. Из $E \mu^{\prime}=$ $H\left(\mu_{+}\right)$и $E \mu^{\prime \prime}=H\left(-\mu_{-}\right)$мы выводим, что $\mu^{\prime} B=\left(\mu_{+}\right) \# B$ и $\mu^{\prime \prime} B=\left(-\mu_{-}\right)^{\#} B$ для каждого $B \in \mathscr{B}_{c}(T, \mathscr{G})$. Из компактной регулярности этих мер заключаем, что $\mu^{\prime}=\left(\left(\mu_{+}\right)^{\#}\right)_{0}$ и $\mu^{\prime \prime}=\left(\left(-\mu_{-}\right)^{\#}\right)_{0}$.

Для ограниченных мер мы можем доказать намного более сильное утверждение, чем в теореме 2 .

Теорема 3. Пусть $(T, \mathscr{G})$ - хаусдорфово пространство. Тогда:

1) отображсние $E_{b}$ имеет единственное расширение до изоморфизма $F_{b}$ : $\mathfrak{R M}_{b}^{w o}(T, \mathscr{G}) \longmapsto \mathfrak{R B}_{b}(T, \mathscr{G})$ межсду данны.ми решеточнымми векторны.ми пространствами такого, что $F_{b} \mu=E_{b}\left(\mu_{+}\right)-E_{b}\left(-\mu_{-}\right)$; 
2) если $\mu \in \mathfrak{R M}_{b}^{w o}(T, \mathscr{G})$ u $\mu=\mu_{1}-\mu_{2}$ для некоторых $\mu_{1}, \mu_{2} \in \mathfrak{R M}_{b}^{w o}(T, \mathscr{G})$, mo $F_{b} \mu=\theta\left(\mu, \mu_{1}, \mu_{2}\right)$.

ДокаЗАТЕЛЬСтво. По предложению из п. $5 \S 1 A \equiv \mathfrak{R M}_{b}^{w o}(T, \mathscr{G})$ является решеточным векторным пространством. По теореме 1 этого пункта $B \equiv \mathfrak{R} \mathfrak{B}_{b}(T, \mathscr{G})$ тоже является решеточным векторным пространством. Поэтому в силу утверждения 2) теоремы 2 и предложения 3.6.1 из [24] отображение $E_{b}$ имеет едиственное расширение до инъективного линейного оператора $F_{b}: A \rightarrow B$ такого, что $F_{b} \mu=E_{b}\left(\mu_{+}\right)-E_{b}\left(-\mu_{-}\right)$.

Пусть $\mu \in A$ и $\mu=\mu_{1}-\mu_{2}$ для некоторых $\mu_{1}, \mu_{2} \in A_{+}$. Тогда $\beta \equiv\left(\mu, \mu_{1}, \mu_{2}\right)$, $\beta_{1} \equiv\left(\mu_{1}, \mu_{1}, 0\right)$ и $\beta_{2} \equiv\left(\mu_{2}, \mu_{2}, 0\right)$ являются борелевско-радоновскими триплетами. Поэтому мы можем рассмотреть радоновские бимеры $\mathfrak{m} \equiv \bar{\beta}, \mathfrak{m}_{1} \equiv \bar{\beta}_{1}$ и $\mathfrak{m}_{2} \equiv \bar{\beta}_{2}$. Ясно, что $\mathfrak{m}_{1}=E_{b} \mu_{1}$ и $\mathfrak{m}_{2}=E_{b} \mu_{2}$. По определению $-\mathfrak{m}_{2}=\theta\left(-\mu_{2}, 0, \mu_{2}\right)$ и $\mathfrak{m}_{1}-\mathfrak{m}_{2}=\theta\left(\mu_{1}-\mu_{2}, \mu_{1}, \mu_{2}\right)=\mathfrak{m}$. В результате $F_{b} \mu=F_{b} \mu_{1}-F_{b} \mu_{2}=E_{b} \mu_{1}-$ $E_{b} \mu_{2}=\mathfrak{m}_{1}-\mathfrak{m}_{2}=\mathfrak{m}$.

По теореме $2 E_{b}$ сохраняет любые порядковые границы. Поэтому $\mu \wedge \nu=0$ в $A_{+}$ влечет $F_{b} \mu \wedge F_{b} \nu=E_{b} \mu \wedge E_{b} \nu=E_{b}(\mu \wedge \nu)=0$. По свойству $14 E(b)$ из [12] $F_{b}$ является решеточным линейным оператором.

Пусть $\mathfrak{m} \in B$. По теореме $2 \mathfrak{m}=E_{b} \mu^{\prime}-E_{b} \mu^{\prime \prime}$ для некоторых $\mu^{\prime}, \mu^{\prime \prime} \in A_{+}$. Рассмотрим меру $\mu \equiv \mu^{\prime}-\mu^{\prime \prime} \in A$. Тогда $\mathfrak{m}=F_{b} \mu^{\prime}-F_{b} \mu^{\prime \prime}=F_{b} \mu$ означает, что $F_{b}$ сюръективно. Таким образом, $F_{b}$ является биективным решеточным линейным оператором. Поэтому $F_{b}-$ необходимый изоморфизм.

СлеДСТВИЕ. Пусть $\mu \in \mathfrak{R M}_{b}^{w o}(T, \mathscr{G})$ u $F_{b} \mu \in \mathfrak{R B}_{b}(T, \mathscr{G})$. Тогда:

1) $p^{\prime} F_{b} \mu=\mu_{+} \quad u \quad p^{\prime \prime} F_{b} \mu=-\mu_{-}$;

2) $F_{b} \mu=\theta\left(\mu, \mu_{+},-\mu_{-}\right)$.

ДоКАЗАТЕЛЬСТВо. В силу предложения из п. $5 \S 1$ мы можем рассмотреть положительные, широкие и ограниченные борелевско-радоновские меры $\mu^{\prime} \equiv \mu_{+}$ и $\mu^{\prime \prime} \equiv-\mu_{-}$. По теореме $3 \mathfrak{m} \equiv F_{b} \mu=E_{b}\left(\mu^{\prime}\right)-E_{b}\left(\mu^{\prime \prime}\right), \mathfrak{m}_{+}=F_{b}\left(\mu_{+}\right)=$ $E_{b}\left(\mu^{\prime}\right)$ и $-\mathfrak{m}_{-}=F_{b}\left(-\mu_{-}\right)=E_{b}\left(\mu^{\prime \prime}\right)$. Поэтому согласно теореме $2 \mu^{\prime}=p^{\prime} \mathfrak{m}$ и $\mu^{\prime \prime}=p^{\prime \prime} \mathfrak{m}$. В результате $\mu=p^{\prime} \mathfrak{m}-p^{\prime \prime} \mathfrak{m}$. Следовательно, в силу предложения 3 $F_{b} \mu=\theta\left(\mu, \mu_{+},-\mu_{-}\right)$.

Отображение $F_{b}: \mathfrak{R M} \mathfrak{M}_{b}^{w o}(T, \mathscr{G}) \nrightarrow \mathfrak{R B}_{b}(T, \mathscr{G})$ назовем каноническим изоморфизмом между решеточным векторным пространством всех иироких ограниченных борелевско-радоновских мер и решеточным векторным пространством всех ограниченных радоновских бимер.

Теоремы 1-3 показывают, что радоновские бимеры образуют естественную решеточную векторную оболочку для семейства всех широких радоновских мер. Более того, в следующем пункте мы установим, что отображения $E$ и $F_{b}$ сохраняют интеграл Лебега.

Теперь мы введем норму в $\mathfrak{R} \mathfrak{B}_{b}(T, \mathscr{G})$ и докажем, что $F_{b}$ сохраняет нормы.

Из теоремы 2 следует, что мы можем корректно определить ранжирование $\rho: \mathfrak{R B _ { b }}(T, \mathscr{G}) \rightarrow \mathbb{R}_{+}$, полагая $\rho \mathfrak{m} \equiv\left(p^{\prime} \mathfrak{m}\right)(T)+\left(p^{\prime \prime} \mathfrak{m}\right)(T)$.

ПРЕДЛОЖЕНИЕ 5. Пусть $(T, \mathscr{G})$ - хаусдорфово пространство. Тогда:

1) $\|\mu\|=\rho\left(H_{b} \mu\right)$ для каждого $\mu \in \mathfrak{R M}_{b}^{n o}(T, \mathscr{G})$; 
2) $\rho$ является монотонной нормой на решеточном векторном пространcmве $\mathfrak{R} \mathfrak{B}_{b}(T, \mathscr{G})$.

ДокаЗАТЕЛЬСтво. 1) Обозначим $\mathfrak{R M}_{b}^{n o}(T, \mathscr{G})$ через $Y$. По предложению 4 из п. $1 \S 2(Y,\|\cdot\|)$ является решеточным векторным пространством с аддитивной нормой. Пусть $\mu \in Y$. Тогда $|\mu|=\mu_{+}+\left(-\mu_{-}\right)$влечет $\|\mu\|=\||\mu|\|=\left\|\mu_{+}\right\|+$ $\left\|-\mu_{-}\right\|$.

Рассмотрим положительные широкие радоновские меры $\nu_{1} \equiv\left(\mu_{+}\right)^{\#}$ и $\nu_{2} \equiv\left(-\mu_{-}\right)^{\#}$. Рассмотрим $\delta$-кольцо $\mathscr{N} \equiv \operatorname{dom}_{f} \nu_{1} \cap \operatorname{dom}_{f} \nu_{2}$ и узкую радоновскую меру $\nu \equiv \nu_{1}\left|\mathscr{N}-\nu_{2}\right| \mathscr{N}$. По определению $H_{b}$ мы имеем $H_{b} \mu=\theta\left(\nu, \nu_{1}, \nu_{2}\right)$. Для каждого $B \in \mathscr{B}_{c}(T, \mathscr{G})$ мы имеем $\nu_{00} B=\nu B=\nu_{1} B-\nu_{2} B=\mu_{+} B-$ $\left(-\mu_{-}\right) B=\mu B . \quad$ Следовательно, по предложению $4 \quad p^{\prime}\left(H_{b} \mu\right)=\left(\left(\mu_{+}\right)^{\#}\right)_{0}$ и $p^{\prime \prime}\left(H_{b} \mu\right)=\left(\left(-\mu_{-}\right)^{\#}\right)_{0}$. В результате $x \equiv\left(p^{\prime} H_{b} \mu\right) T=\left(\mu_{+}\right)^{\#} T$ и $y \equiv\left(p^{\prime \prime} H_{b} \mu\right) T=$ $\left(-\mu_{-}\right) \# T$. По предложениям 1 и 2 из п. $1 \S 2 \mu_{+}$и $\left(\mu_{+}\right)^{\#}$ компактно регулярны. Поэтому

$$
x=\sup \left\{\left(\mu_{+}\right)^{\#} C \mid C \in \mathscr{C}\right\}=\sup \left\{\mu_{+} C \mid C \in \mathscr{C}\right\}=\mu_{+} T=\left\|\mu_{+}\right\| .
$$

Точно так же $y=\left\|-\mu_{-}\right\|$. В заключение мы получаем $\|\mu\|=x+y=\rho\left(H_{b} \mu\right)$.

2) Обозначим $\mathfrak{R} \mathfrak{B}_{b}(T, \mathscr{G})$ через $Z$. По теореме 1 этого пункта $H_{b}: Y \longmapsto Z$ является изоморфизмом данных решеточных векторных пространств. Поэтому

$$
\rho\left(z_{1}+z_{2}\right)=\left\|H_{b}^{-1} z_{1}+H_{b}^{-1} z_{2}\right\| \leqslant\left\|H_{b}^{-1} z_{1}\right\|+\left\|H_{b}^{-1} z_{2}\right\|=\rho z_{1}+\rho z_{2}
$$

для каждых $z_{1}, z_{2} \in Z$. Если $a \in \mathbb{R}$ и $z \in Z$, то

$$
\rho(a z)=\left\|H_{b}^{-1}(a z)\right\|=|a|\left\|H_{b}^{-1} z\right\|=|a| \rho z .
$$

Если $\rho z=0$, то $\left\|H_{b}^{-1} z\right\|=0$ влечет $H_{b}^{-1} z=0$, откуда $z=0$. Таким образом, $\rho$ является нормой на $Z$. Если $\left|z_{1}\right| \leqslant\left|z_{2}\right|$ в $Z$, то по утверждению 1$)\left|H_{b}^{-1} z_{1}\right| \leqslant$ $\left|H_{b}^{-1} z_{2}\right|$ влечет $\rho z_{1}=\left\|H_{b}^{-1} z_{1}\right\| \leqslant\left\|H_{b}^{-1} z_{2}\right\|=\rho z_{2}$. Поэтому норма $\rho$ монотонна.

Далее мы будем обозначать $\rho$ через $\|\cdot\|$.

СлЕДСТВИЕ. 1) $\left(\mathfrak{R B}_{b}(T, \mathscr{G}),\|\cdot\|\right)$ является решеточным векторным пространством с аддитивной нормой.

2) $H_{b}:\left(\mathfrak{R M}_{b}^{\text {no }}(T, \mathscr{G}),\|\cdot\|\right) \longmapsto\left(\mathfrak{R B}_{b}(T, \mathscr{G}),\|\cdot\|\right)$ является ( изометрическим) изоморфизмом данных нормированных решеточных векторных пространств.

ДокАЗАТЕЛЬСТво. Будем использовать обозначения из доказательства предложения 5. Согласно этому предложению $(Z,\|\cdot\|)$ является нормированным решеточным векторным пространством и $H_{b}:(Y,\|\cdot\|) \longmapsto(Z,\|\cdot\|)$ является изометрическим изоморфизмом данных решеточных векторных пространств. Значит, $H_{b}-$ необходимый изоморфизм. По предложению 4 из п. $1 \S 2(Y,\|\cdot\|)$ обладает аддитивной нормой. Используя упомянутый изоморфизм $H_{b}$, мы проверяем, что $(Z,\|\cdot\|)$ также обладает аддитивной нормой.

В заключение мы свяжем все рассмотренные биекции и изоморфизмы. 
ПРЕДЛОЖЕНИЕ 6 . Пусть $(T, \mathscr{G})$ - хаусдорфово пространство. Тогда:

1) $H \mu=(E \circ R) \mu$ для каждого $\mu \in \mathfrak{R M}^{n o}(T, \mathscr{G})_{+}$;

2) $H_{b}=F_{b} \circ S_{b}$.

ДокаЗАТЕльство. 1) Для $\mu$ рассмотрим меры $\varkappa_{1} \equiv \mu$ и $\varkappa_{2} \equiv 0$. Тогда $\varkappa_{1}^{\#}=\mu^{\#}$ и $\varkappa_{2}^{\#}=0$. Мы можем предположить, что $\operatorname{dom} \varkappa_{2}^{\#}=\operatorname{dom} \varkappa_{1}^{\#}=\operatorname{dom} \mu^{\#}$. Рассмотрим на $\delta$-кольце $\operatorname{dom}_{f} \mu^{\#}$ положительную узкую радоновскую меру $\varkappa=$ $\varkappa_{1}^{\#} \mid \operatorname{dom}_{f} \mu^{\#}=\left(\mu^{\#}\right)_{f}$. Тогда по определению $H$ мы имеем $H \mu=\theta\left(\left(\mu^{\#}\right)_{f}, \mu^{\#}, 0\right)$.

$\mathrm{C}$ другой стороны, $R \mu=\left(\mu^{\#}\right)_{0}$ и $E R \mu=\theta\left(\left(\left(\mu^{\#}\right)_{0}\right)_{f},\left(\mu^{\#}\right)_{0}, 0\right)$. Так как $\left(\mu^{\#}\right)_{f} B=\mu B=\left(\left(\mu^{\#}\right)_{0}\right)_{f} B$ для каждого $B \in \mathscr{B}_{c}(T, \mathscr{G})$, мы заключаем, что $H \mu=E R \mu$.

Если $\mu$ является ограниченной положительной мерой, то $H_{b} \mu=E_{b} R_{b} \mu$.

2) Если $\mu \in \mathfrak{R M}_{b}^{n o}(T, \mathscr{G})$, то по теореме 1 из п. $1 \S 2$ и теоремам 1 и 3 этого пункта $H_{b} \mu=H_{b}\left(\mu_{+}\right)-H_{b}\left(-\mu_{-}\right)=E_{b} R_{b}\left(\mu_{+}\right)-E_{b} R_{b}\left(-\mu_{-}\right)=F_{b} S_{b}\left(\mu_{+}\right)-$ $F_{b} S_{b}\left(-\mu_{-}\right)=F_{b} S_{b} \mu$.

СледСТВИЕ. $F_{b}:\left(\mathfrak{R M}_{b}^{w o}(T, \mathscr{G}),\|\cdot\|\right) \longmapsto\left(\mathfrak{R B}_{b}(T, \mathscr{G}),\|\cdot\|\right)$ является $($ изометрическим) изоморфизмом данных нормированных решеточных векторных пространств.

ДокАЗАТЕЛЬСтво. Если $\mu$ - широкая ограниченная радоновская мера, то по следствию к предложению $5\left\|S_{b}^{-1} \mu\right\|=\left\|H_{b} S_{b}^{-1} \mu\right\|=\left\|F_{b} \mu\right\|$. Сходно, по предложению 4 из п. $1 \S 2\left\|S_{b}^{-1} \mu\right\|=\|\mu\|$. В результате $\|\mu\|=\left\|F_{b} \mu\right\|$.

3. Интеграл Лебега относительно радоновских бимер. Теперь мы введем интегрирование относительно радоновской бимеры. Для каждого радоновского триплета $\beta \equiv\left(\mu, \mu_{1}, \mu_{2}\right)$ рассмотрим соответствуюшие $\sigma$-алгебру $\operatorname{dom} \beta \equiv$ $\operatorname{dom} \mu_{1} \cap \operatorname{dom} \mu_{2}$ и $\delta$-кольцо $\operatorname{dom}_{f} \beta \equiv \operatorname{dom}_{f} \mu_{1} \cap \operatorname{dom}_{f} \mu_{2}$.

Рассмотрим также широкие борелевско-радоновские меры $\lambda_{1} \equiv\left(\mu_{1}\right)_{0}$ и $\lambda_{2} \equiv$ $\left(\mu_{2}\right)_{0}$ и полные сильно насьшенные радоновские меры $\nu_{1} \equiv \mu_{1}^{\#}$ и $\nu_{2} \equiv \mu_{2}^{\#}$ из леммы п. $5 \S 1$. Рассмотрим $\delta$-кольца $\mathscr{L} \equiv \operatorname{dom}_{f} \lambda_{1} \cap \operatorname{dom}_{f} \lambda_{2}$ и $\mathscr{N} \equiv \operatorname{dom}_{f} \nu_{1} \cap$ $\operatorname{dom}_{f} \nu_{2}$ и соответствуюшие конечные меры $\lambda \equiv \lambda_{1}\left|\mathscr{L}-\lambda_{2}\right| \mathscr{L}$ и $\nu \equiv \nu_{1} \mid \mathscr{N}-$ $\nu_{2} \mid \mathcal{N}$. Тогда мы получим борелевско-радоновский триплет $\beta_{0} \equiv\left(\lambda, \lambda_{1}, \lambda_{2}\right)$ и расширенный радоновский триплет $\beta_{e} \equiv\left(\nu, \nu_{1}, \nu_{2}\right)$.

Лемма 1. Если $\beta \in R(T, \mathscr{G}), \operatorname{mo} \beta_{0} \in R^{0}(T, \mathscr{G}), \beta_{e} \in R^{e}(T, \mathscr{G}) u \beta \sim \beta_{0} \sim \beta_{e}$.

ДоказАтельство. Если $B \in \mathscr{B}_{c}(T, \mathscr{G})$, то $\mu B=\mu_{1} B-\mu_{2} B=\lambda_{1} B-$ $\lambda_{2} B=\lambda B$ и $\mu B=\mu_{1} B-\mu_{2} B=\nu_{1} B-\nu_{2} B=\nu B$. Это означает, что $\beta \sim \beta_{0}$ и $\beta \sim \beta_{e}$.

СлЕДСТВИЕ. Для каждой радоновской бимерь $\mathfrak{m}$ существуют радоновские триплеты $\alpha \in R^{0}(T, \mathscr{G})$ u $\gamma \in R^{e}(T, \mathscr{G})$ такие, ито $\mathfrak{m}=\bar{\alpha}=\bar{\gamma}$.

Лемма 2. Пусть $\beta \equiv\left(\mu, \mu_{1}, \mu_{2}\right) u \gamma \equiv\left(\nu, \nu_{1}, \nu_{2}\right)-$ радоновские триплеть u $\beta \sim \gamma$. Тогда для каждой $\sigma$-алгебры $\mathscr{L} \subset \operatorname{dom} \beta \cap \operatorname{dom} \gamma$ такой, что $\mathscr{B}_{c}(T, \mathscr{G}) \subset \mathscr{L}$, и каждой функиии $f \in \operatorname{MI}\left(T, \mathscr{L}, \mu_{1} \mid \mathscr{L}\right) \cap \mathrm{MI}\left(T, \mathscr{L}, \mu_{2} \mid \mathscr{L}\right) \cap$ $\operatorname{MI}\left(T, \mathscr{L}, \nu_{1} \mid \mathscr{L}\right) \cap \operatorname{MI}\left(T, \mathscr{L}, \nu_{2} \mid \mathscr{L}\right)$ справедливо $\int f d \mu_{1}-\int f d \mu_{2}=\int f d \nu_{1}-$ $\int f d \nu_{2}$. 
ДокАЗАТЕЛЬСтво. Обозначим ограничения мер $\mu_{1}, \mu_{2}, \nu_{1}, \nu_{2}$ на $\sigma$-алгебру $\mathscr{L}$ через $\mu_{1}^{\prime} \mu_{2}^{\prime}, \nu_{1}^{\prime}$ и $\nu_{2}^{\prime}$ соответственно. По определению $\mu_{1}^{\prime} B-\mu_{2}^{\prime} B=\mu B=\nu B=$ $\nu_{1}^{\prime} B-\nu_{2}^{\prime} B$ для каждого $B \in \mathscr{B}_{c}(T, \mathscr{G})$. Рассмотрим положительные широкие радоновские меры $\pi \equiv \mu_{1}^{\prime}+\nu_{2}^{\prime}$ и $\varkappa \equiv \mu_{2}^{\prime}+\nu_{1}^{\prime}$ на $\mathscr{L}$. Тогда $\pi C=\varkappa C$ для каждого компактного множества $C$. Из компактной регулярности этих мер мы выводим, что $\pi=\varkappa$. Поэтому $X \equiv \mathrm{MI}\left(T, \mathscr{L}, \mu_{1}^{\prime}\right) \cap \mathrm{MI}\left(T, \mathscr{L}, \nu_{2}^{\prime}\right)=\mathrm{MI}(T, \mathscr{L}, \pi)=\mathrm{MI}(T, \mathscr{L}, \varkappa)=$ $\operatorname{MI}\left(T, \mathscr{L}, \mu_{2}^{\prime}\right) \cap \operatorname{MI}\left(T, \mathscr{L}, \nu_{1}^{\prime}\right) \equiv Y$ и $\int f d \mu_{1}^{\prime}+\int f d \nu_{2}^{\prime}=\int f d \pi=\int f d \varkappa=\int f d \mu_{2}^{\prime}+$ $\int f d \nu_{1}^{\prime}$ для каждой функции $f$ из этого семейства.

Если $f \in X \cap Y$, то $f$ является также интегрируемой относительно мер $\mu_{1}, \mu_{2}, \nu_{1}$ и $\nu_{2}$ и $\int f d \mu_{1}^{\prime}=\int f d \mu_{1}, \int f d \mu_{2}^{\prime}=\int f d \mu_{2}, \int f d \nu_{1}^{\prime}=\int f d \nu_{1}$ и $\int f d \nu_{2}^{\prime}=\int f d \nu_{2}$. В результате для $f \in X \cap Y$ мы получаем $\int f d \mu_{1}-\int f d \mu_{2}=\int f d \nu_{1}-\int f d \nu_{2}$.

Согласно лемме 2 естественно рассмотреть для каждой положительной широкой борелевско-радоновской меры $\mu$ семейства $\mathrm{BM}(T, \mathscr{G}) \equiv M(T, \mathscr{B}(T, \mathscr{G}))$ и $\mathrm{BI}(T, \mathscr{G}, \mu) \equiv \operatorname{MI}(T, \mathscr{B}(T, \mathscr{G}), \mu)$ всех борелевско-измеримых функций и всех борелевско-измеримых н-интегрируемых функций соответственно.

СЛЕДСТВИЕ 1 . Пусть $\beta \equiv\left(\mu, \mu_{1}, \mu_{2}\right) u \gamma \equiv\left(\nu, \nu_{1}, \nu_{2}\right)-$ радоновские триплеты и $\beta \sim \gamma$. Тогда $\int f d \mu_{1}-\int f d \mu_{2}=\int f d \nu_{1}-\int f d \nu_{2}$ для каждой функиии $f \in \mathrm{BI}\left(T, \mathscr{G},\left(\mu_{1}\right)_{0}\right) \cap \mathrm{BI}\left(T, \mathscr{G},\left(\mu_{2}\right)_{0}\right) \cap \mathrm{BI}\left(T, \mathscr{G},\left(\nu_{1}\right)_{0}\right) \cap \mathrm{BI}\left(T, \mathscr{G},\left(\nu_{2}\right)_{0}\right)$.

СЛЕДСТВИЕ 2 . Пусть $\beta \equiv\left(\mu, \mu_{1}, \mu_{2}\right) u \gamma \equiv\left(\nu, \nu_{1}, \nu_{2}\right)$ - борелевско-радоновские триплетьи и $\beta \sim \gamma$. Тогда $\int f d \mu_{1}-\int f d \mu_{2}=\int f d \nu_{1}-\int f d \nu_{2}$ для каждой функиии $f \in \mathrm{BI}\left(T, \mathscr{G}, \mu_{1}\right) \cap \mathrm{BI}\left(T, \mathscr{G}, \mu_{2}\right) \cap \mathrm{BI}\left(T, \mathscr{G}, \nu_{1}\right) \cap \mathrm{BI}\left(T, \mathscr{G}, \nu_{2}\right)$.

Пусть теперь $\mathfrak{m}$ - радоновская бимера. Согласно следствию из леммы 1 этого пункта $\mathfrak{m} \cap R^{0}(T, \mathscr{G}) \neq \varnothing$. Поэтому мы можем рассмотреть для $\mathfrak{m}$ семейство $\mathrm{WI}(T, \mathscr{G}, \mathfrak{m}) \equiv \cap\left\{\operatorname{BI}\left(T, \mathscr{G}, \mu_{1}\right) \cap \mathrm{BI}\left(T, \mathscr{G}, \mu_{2}\right) \mid\left(\mu, \mu_{1}, \mu_{2}\right) \in \mathfrak{m} \cap R^{0}(T, \mathscr{G})\right\}$ всех борелевско-измеримых функций, которые интегрируемы относительно (широких) положительных борелевско-радоновских мер $\mu_{1}$ и $\mu_{2}$ из каждого борелевско-радоновского триплета $\left(\mu, \mu_{1}, \mu_{2}\right)$ из бимеры $\mathfrak{m}$.

Рассмотрим для $\mathfrak{m}$ также более широкое семейство $\mathrm{BI}(T, \mathscr{G}, \mathfrak{m}) \equiv \mathrm{BI}\left(T, \mathscr{G}, p^{\prime} \mathfrak{m}\right) \cap$ $\mathrm{BI}\left(T, \mathscr{G}, p^{\prime \prime} \mathfrak{m}\right)$.

Каждую функцию из семейства $\mathrm{BI}(T, \mathscr{G}, \mathfrak{m})$ назовем борелевско-измеримой интегрируемой по Лебегу относительно бимерь $\mathfrak{m}$. А каждую функцию из семейства $\mathrm{WI}(T, \mathscr{G}, \mathfrak{m})$ назовем борелевско-измеримой вполне интегрируемой по Лебегу относительно бимеры $\mathfrak{m}$.

Число $\int f d p^{\prime} \mathfrak{m}-\int f d p^{\prime \prime} \mathfrak{m}$ назовем интегралом (Лебега) от функиии $f \in$ $\mathrm{BI}(T, \mathscr{G}, \mathfrak{m})$ над топологическим биизмеримымм пространством $(T, \mathscr{G}, \mathfrak{m})$ и обозначим через $\int f d \mathfrak{m}$.

ЛЕмма 3. Пусть $\mathfrak{m} \in \mathfrak{R} \mathfrak{B}(T, \mathscr{G}) u\left(\nu, \nu_{1}, \nu_{2}\right) \in \mathfrak{m} \cap R^{0}(T, \mathscr{G})$. Тогда $\int f d \mathfrak{m}=$ $\int f d \nu_{1}-\int f d \nu_{2}$ для каждой функиии $f \in \mathrm{WI}(T, \mathscr{G}, \mathfrak{m})$.

ДокАЗАТЕЛЬСТво. Рассмотрим $\delta$-кольцо $\mathscr{M} \equiv \operatorname{dom}_{f}\left(p^{\prime} \mathfrak{m}\right) \cap \operatorname{dom}_{f}\left(p^{\prime \prime} \mathfrak{m}\right)$ и узкую борелевско-радоновскую меру $\mu \equiv p^{\prime} \mathfrak{m}\left|\mathscr{M}-p^{\prime \prime} \mathfrak{m}\right| \mathscr{M}$. По предложению 3 из п. $2 \S 2\left(\mu, p^{\prime} \mathfrak{m}, p^{\prime \prime} \mathfrak{m}\right) \in \mathfrak{m} \cap R^{0}(T, \mathscr{G})$. Поэтому по следствию 2 из леммы 2 этого пункта $\int f d \mathfrak{m}=\int f d \nu_{1}-\int f d \nu_{2}$. 
ПРЕДЛОЖЕНИЕ 1. Пусть $(T, \mathscr{G})$ - хаусдорфово пространство.

1) $E с л и \mu \in \mathfrak{R M}^{w o}(T, \mathscr{G})_{0}$, mo $\int f d \mu=\int f d E \mu$ для каждой функции $f \in$ $\mathrm{BI}(T, \mathscr{G}, \mu)=\mathrm{BI}(T, \mathscr{G}, E \mu)$.

2) Ecли $\mu \in \mathfrak{R M}_{b}^{w o}(T, \mathscr{G})$, mo $\int f d \mu=\int f d F_{b} \mu$ для каждой функиии $f \in$ $\mathrm{BI}(T, \mathscr{G}, \mu)=\mathrm{BI}\left(T, \mathscr{G}, F_{b} \mu\right)$.

ДоказАТЕЛЬСтво. 1) Обозначим $E \mu$ через $\mathfrak{m}$. В силу теоремы 2 из п. $2 \S 2$ $\mathfrak{m} \geqslant 0$. Поэтому $\mathfrak{m}_{+}=E \mu,-\mathfrak{m}_{-}=0=E 0$ и $\mathfrak{m}=E \mu-E 0$. Следовательно, по теореме 2 из п. $2 \S 2 \mu=p^{\prime} \mathfrak{m}$ и $0=p^{\prime \prime} \mathfrak{m}$. Это влечет $\mathrm{BI}(T, \mathscr{G}, \mu)=\mathrm{BI}\left(T, \mathscr{G}, p^{\prime} \mathfrak{m}\right) \cap$ $\mathrm{BI}\left(T, \mathscr{G}, p^{\prime \prime} \mathfrak{m}\right) \equiv \mathrm{BI}(T, \mathscr{G}, \mathfrak{m})$ и $\int f d \mu=\int f d p^{\prime} \mathfrak{m}-\int f d p^{\prime \prime} \mathfrak{m} \equiv \int f d \mathfrak{m}$ для каждого $f \in \mathrm{BI}(T, \mathscr{G}, \mu)$.

2) Обозначим $F_{b} \mu$ через $\mathfrak{m}$. По следствию из теоремы 3 п. $2 \S 2 p^{\prime} \mathfrak{m}=\mu_{+}$и $p^{\prime \prime} \mathfrak{m}=-\mu_{-}$. Поэтому $\mathrm{BI}(T, \mathscr{G}, \mu) \equiv \mathrm{BI}\left(T, \mathscr{G}, \mu_{+}\right) \cap \mathrm{BI}\left(T, \mathscr{G},-\mu_{-}\right)=\mathrm{BI}\left(T, \mathscr{G}, p^{\prime} \mathfrak{m}\right) \cap$ $\mathrm{BI}\left(T, \mathscr{G}_{,} p^{\prime \prime} \mathfrak{m}\right) \equiv \mathrm{BI}(T, \mathscr{G}, \mathfrak{m})$ и $\int f d \mu \equiv \int f d\left(\mu_{+}\right)-\int f d\left(-\mu_{-}\right)=\int f d p^{\prime} \mathfrak{m}-$ $\int f d p^{\prime \prime} \mathfrak{m} \equiv \int f d \mathfrak{m}$.

Таким образом, биекция $E: \mathfrak{R M}^{w o}(T, \mathscr{G})_{0} \longmapsto \mathfrak{R B}(T, \mathscr{G})_{+}$и изоморфизм $F_{b}$ : $\mathfrak{R M}_{b}^{w o}(T, \mathscr{G}) \longmapsto \mathfrak{R B}_{b}(T, \mathscr{G})$ сохраняют интеграл Лебега. Это показывает, что определения интегралов Лебега для широких мер (см. п. 1 из $§ 1$ ) и для бимер согласованы друг с другом.

ПРЕДЛОЖЕНИЕ 2. Пусть $(T, \mathscr{G})-$ хаусдорфово пространство, $\mathfrak{m}, \mathfrak{n} \in$ $\mathfrak{R B}(T, \mathscr{G}) \quad$ u $x, y \in \mathbb{R} . \quad$ Тогда $\int f d(x \mathfrak{m}+y \mathfrak{n})=x \int f d \mathfrak{m}+y \int f d \mathfrak{n}$ для каждой функиии $f \in \mathrm{WI}(T, \mathscr{G}, \mathfrak{m}) \cap \mathrm{WI}(T, \mathscr{G}, \mathfrak{n}) \cap \mathrm{WI}(T, \mathscr{G}, x \mathfrak{m}+y \mathfrak{n})$.

ДокАЗАТЕЛЬСТво. Возьмем любые борелевско-радоновские триплеты $\beta \equiv$ $\left(\mu, \mu_{1}, \mu_{2}\right) \in \mathfrak{m}$ и $\gamma \equiv\left(\nu, \nu_{1}, \nu_{2}\right) \in \mathfrak{n}$. Предположим, что $x \geqslant 0$ и $y<0$. По определению $x \mathfrak{m}=\theta\left(x \mu, x \mu_{1}, x \mu_{2}\right)$ и $y \mathfrak{n}=\theta\left(y \nu,-y \nu_{2},-y \nu_{1}\right)$. Рассмотрим $\delta$-кольцо $\mathscr{L} \equiv \operatorname{dom}_{f} \beta \cap \operatorname{dom}_{f} \gamma$. Рассмотрим положительные широкие борелевско-радоновские меры $\lambda_{1} \equiv x \mu_{1}+\left(-y \nu_{2}\right)$ и $\lambda_{2} \equiv x \mu_{2}+\left(-y \nu_{1}\right)$ и узкую радоновскую меру $\lambda \equiv x \mu|\mathscr{L}+y \nu| \mathscr{L}$. Тогда для борелевско-радоновского триплета $\alpha \equiv\left(\lambda, \lambda_{1}, \lambda_{2}\right)$ имеем $x \mathfrak{m}+y \mathfrak{n}=\bar{\alpha}$.

Возьмем любую функцию $f$, как в утверждении предложения 2 . Тогда по лемме $3 a \equiv \int f d \mathfrak{m}=\int f d \mu_{1}-\int f d \mu_{2}, \quad b \equiv \int f d \mathfrak{n}=\int f d \nu_{1}-\int f d \nu_{2}$ и $c \equiv \int f d(x \mathfrak{m}+y \mathfrak{n})=\int f d \lambda_{1}-\int f d \lambda_{2}$. По линейности интеграла Лебега относительно положительных широких мер и положительных чисел мы получаем $c=x \int f d \mu_{1}-y \int f d \nu_{2}-x \int f d \mu_{2}+y \int f d \nu_{1}=x a+y b$.

Обозначим через $B M_{b c}(T, \mathscr{G})$ семейство всех ограниченных борелевско-измеримых функций с компактными носителями.

ПРЕДЛОЖЕНИЕ 3. Пусть $(T, \mathscr{G})$ - хаусдорфово пространство. Тогда

$$
B M_{b c}(T, \mathscr{G}) \subset \mathrm{WI}(T, \mathscr{G}, \mathfrak{m})
$$

для каждой бимеры $\mathfrak{m} \in \mathfrak{R} \mathfrak{B}(T, \mathscr{G})$. 
ДоКАЗАТЕЛЬСтво. Если $f \in B M_{b c}(T, \mathscr{G})$, то множество $S \equiv \operatorname{supp} f$ является компактным. Пусть $\left(\mu, \mu_{1}, \mu_{2}\right) \in \mathfrak{m} \cap R^{0}(T, \mathscr{G})$. Тогда $\mu_{1} S<\infty$ и $\mu_{2} S<\infty$. Поэтому $\int f_{+} d \mu_{1} \leqslant \int\|f\|_{u} \chi(S) d \mu_{1}=\|f\|_{u} \mu_{1} S<\infty$. Это означает, что $f_{+} \in$ $\mathrm{BI}\left(T, \mathscr{G}, \mu_{1}\right)$. То же самое справедливо для $-f_{-}$. По определению из п. $1 \S 1$ $f \in \mathrm{BI}\left(T, \mathscr{G}, \mu_{1}\right)$. Аналогично $f \in \mathrm{BI}\left(T, \mathscr{G}, \mu_{2}\right)$. В результате мы заключаем, что $f \in \mathrm{WI}(T, \mathscr{G}, \mathfrak{m})$.

4. Метаполунепрерывные функции и тонкие функционалы. В работах [31]-[33] был введен новый класс функций, сушественно расширяюший класс измеримых функций. Мы будем использовать функции из этого класса для решения проблемы радоновского представления.

Если $f \in F(T)$ и $S \subset T$, то число $\omega(f, S) \equiv \sup \{|f(s)-f(t)| \mid s, t \in S\}$ назьвается колебанием функиии $f$ на множестве $S$.

Пусть $\mathscr{S}$ - некоторый ансамбль подмножеств множества $T$. Функцию $f \in F(T)$ будем называть равномерной (счетно-равномерной, конечно-равномерной) относительно $\mathscr{S}$, если для каждого $\varepsilon>0$ существует покрытие (счетное покрытие, конечное покрытие) $\varkappa \equiv\left(S_{i} \in \mathscr{S} \mid i \in I\right)$ множества $T$ такое, что $\omega\left(f, S_{i}\right)<\varepsilon$ для каждого $i \in I$. Семейства всех равномерных, всех счетно-равномерных и всех конечно-равномерных относительно $\mathscr{S}$ функций на множестве $T$ обозначим через $U(T, \mathscr{S}), U^{c}(T, \mathscr{S})$ и $U^{f}(T, \mathscr{S})$ соответственно. Ясно, что $U^{f}(T, \mathscr{S}) \subset F_{b}(T) \cap$ $U^{c}(T, \mathscr{S}) \equiv U_{b}^{c}(T, \mathscr{S}) \subset F_{b}(T) \cap U(T, \mathscr{S}) \equiv U_{b}(T, \mathscr{S})$ и $U^{c}(T, \mathscr{S}) \subset U(T, \mathscr{S})$.

Ансамбль $\mathscr{S}$ называется основой [33], если он содержит подмножества $\varnothing$ и $T$ и замкнут относительно конечных пересечений.

ПРЕДЛОЖЕНИЕ 1. Пусть $T$ - множество и $\mathscr{S}$ - основа на T. Тогда $U(T, \mathscr{S})$, $U^{c}(T, \mathscr{S})$ и $U^{f}(T, \mathscr{S})$ являются решеточными векторными пространствами.

ДокаЗАтЕльСтво. Пусть $f, g \in U(T, \mathscr{S})$ и $x, y \in \mathbb{R} \backslash\{0\}$. Возьмем $\varepsilon>0$. Тогда для $\varepsilon_{1} \equiv \varepsilon /(2|x|)$ и $\varepsilon_{2} \equiv \varepsilon /(2|y|)$ найдутся покрытия $\pi \equiv\left(P_{i} \in \mathscr{S} \mid i \in I\right)$ и $\rho \equiv\left(R_{j} \in \mathscr{S} \mid j \in J\right)$ такие, что $\omega\left(f, P_{i}\right)<\varepsilon_{1}$ и $\omega\left(g, R_{j}\right)<\varepsilon_{2}$ для всех $i, j$. Возьмем покрытие $\varkappa \equiv\left(S_{k} \mid k \in K\right)$ такое, что $K \equiv I \times J$ и $S_{k} \equiv P_{i} \cap R_{j}$ для $k \equiv(i, j)$. Тогда для $h \equiv x f+y g$ мы имеем

$$
|h(s)-h(t)| \leqslant|x||f(s)-f(t)|+|y||g(s)-g(t)|<|x| \varepsilon_{1}+|y| \varepsilon_{2}=\varepsilon
$$

для всех $s, t \in S_{k}$ и $k \in K$. Следовательно, $\omega\left(h, S_{k}\right)<\varepsilon$ для каждого $k \in K$. Значит, $x f+y g \in U(T, \mathscr{S})$.

Далее, для $\varepsilon_{1} \equiv \varepsilon / 2$ и $\varepsilon_{2} \equiv \varepsilon / 2$ найдутся покрытия $\pi$ и $\rho$ такие же, как в предыдущем абзаце. Возьмем такое же покрытие $\varkappa$. Тогда для $k \in K$ и $s, t \in S_{k}$ мы имеем

$$
\begin{aligned}
|f(t) \vee g(t)-f(s) \vee g(s)| & \leqslant|f(t) \vee g(t)-f(t) \vee g(s)|+|f(t) \vee g(s)-f(s) \vee g(s)| \\
& \leqslant|g(t)-g(s)|+|f(t)-f(s)| \leqslant \varepsilon_{1}+\varepsilon_{2}=\varepsilon .
\end{aligned}
$$

Следовательно, $\omega\left(f \vee g, S_{k}\right)<\varepsilon$ для каждого $k \in K$. Значит, $f \vee g \in U(T, \mathscr{S})$. Точно так же $f \wedge g \in U(T, \mathscr{S})$.

На хаусдорфовом пространстве $(T, \mathscr{G})$ рассмотрим семейство $S C_{b}^{l}(T, \mathscr{G})$ всех ограниченных полунепрерывных снизу функций $f$, т.е. таких, что $f^{-1}[] x, \infty[] \in \mathscr{G}$ 
для каждого $x \in \mathbb{R}$. Так как это семейство не является векторным пространством, Хаусдорф $[34, \S 37.5]$ рассмотрел семейство $S C^{b}(T, \mathscr{G}) \equiv\left\{f-g \mid f, g \in S C_{b}^{l}(T, \mathscr{G})\right\}$. Элементы из $S C^{b}(T, \mathscr{G})$ естественно называть ограниченными полунепрерывными функциями.

Лемма 1. Семейство $S C^{b}(T, \mathscr{G})$ является решеточным векторным пространством.

ДокАЗАТЕЛЬСТво. Пусть $f=f_{1}-f_{2}, g=g_{1}-g_{2}$ и $x \in \mathbb{R}$. Если $x>0$, то $x f=x f_{1}-x f_{2} \in S C^{b}(T, \mathscr{G})$. Если $x<0$, то $x f=(-x) f_{2}-(-x) f_{1} \in S C^{b}(T, \mathscr{G})$. Аналогично $f+g=\left(f_{1}+g_{1}\right)-\left(f_{2}+g_{2}\right) \in S C^{b}(T, \mathscr{G})$.

Далее, $f \wedge g=\left(f_{1}-f_{2}\right) \wedge\left(g_{1}-g_{2}\right)=f_{1} \wedge\left(g_{1}+f_{2}-g_{2}\right)-f_{2}=\left(f_{1}+g_{2}\right) \wedge\left(g_{1}+f_{2}\right)-$ $\left(g_{2}+f_{2}\right) \in S C^{b}(T, \mathscr{G})$. Наконец, $f \vee g=\left(f_{1}+g_{2}\right) \vee\left(g_{1}+f_{2}\right)-\left(g_{2}+f_{2}\right) \in S C^{b}(T, \mathscr{G})$.

Таким образом, семейство $S C^{b}(T, \mathscr{G})$ обладает достаточно хорошими свойствами. Однако Серпинский [35] установил, что оно не является замкнутым в $F_{b}(T)$ относительно равномерной сходимости последовательностей, и рассмотрел равномерное замыкание $S(T, \mathscr{G})$ семейства $S C^{b}(T, \mathscr{G})$ в нормированном пространстве $\left(F_{b}(T),\|\cdot\|_{u}\right)$. Долгое время не было известно никакого функционального описания семейства $S(T, \mathscr{G})$. Оно было дано в работах [36] и [37]. В работе [38] семейство Хаусдорфа-Серпинского $S(T, \mathscr{G})$ было названо семейством метаполунепрерывных функций. Приведем сначала функциональное описание $S(T, \mathscr{G})$.

Рассмотрим алгебру $\mathscr{A}(T, \mathscr{G})$ всех александровских подмножеств из $T$, порожденную решеткой $\mathscr{G}$. Эта алгебра широко использовалась Александровым в работе [26].

ПРеДЛОЖЕНИЕ 2. Пусть $(T, \mathscr{G})$ - хаусдорфово пространство. Тогда $A \in$ $\mathscr{A}(T, \mathscr{G})$, если и только если $A=\cup\left\{G_{i} \cap F_{i} \mid i \in I\right\}$ для некоторых конечных коллекиий $\left(G_{i} \in \mathscr{G} \mid i \in I\right)$ u $\left(F_{i} \in \mathscr{F} \mid i \in I\right)$.

ДокАЗАТЕЛЬСТво. Ясно, что ансамбль $\mathscr{E}$ всех множеств $A$ такого вида замкнут относительно конечных объединений и содержит множество $T$. Пусть $A=\cup\left\{G_{i}^{\prime} \cap\right.$ $\left.F_{i}^{\prime} \mid i \in I\right\}$. Рассмотрим множества $G_{i} \equiv T \backslash F_{i}^{\prime}$ и $F_{i} \equiv T \backslash G_{i}^{\prime}$. Тогда $T \backslash A=$ $\cap\left\{G_{i} \cup F_{i} \mid i \in I\right\}$. Ясно, что

$$
T \backslash A \subset \cup\left\{\cap\left\{G_{m} \mid m \in M\right\} \cap \cap\left\{F_{l} \mid l \in L\right\} \mid L \cup M=I\right\} \equiv B .
$$

Обратно: рассмотрим множество $Q \equiv \cap\left\{G_{m} \mid m \in M\right\} \cap \cap\left\{F_{l} \mid l \in L\right\}$. Возьмем произвольное $i \in I$. Если $i=l$, то $Q \subset F_{l} \subset F_{i} \cup G_{i}$. Если $i=m$, то $Q \subset G_{m} \subset$ $F_{i} \cup G_{i}$. Поэтому $Q \subset T \backslash A$. Таким образом, $T \backslash A=B \in \mathscr{E}$. Следовательно, $\mathscr{E}$ является алгеброй. Очевидно, что $\mathscr{E}=\mathscr{A}(T, \mathscr{G})$.

Обозначим через $\mathrm{St}(T, \mathscr{A}(T, \mathscr{G}))$ решеточное векторное пространство всех ступенчатых функций $f \equiv \sum\left(x_{k} \chi\left(A_{k}\right) \mid k \in K\right)$ относительно алгебры $\mathscr{A}(T, \mathscr{G})$.

ЛЕммa 2. St $(T, \mathscr{A}(T, \mathscr{G})) \subset S C^{b}(T, \mathscr{G})$.

ДокаЗАТЕЛЬСТво. Пусть $A \in \mathscr{A}(T, \mathscr{G})$. По предложению $2 A=\cup\left\{G_{i} \cap\right.$ $\left.F_{i} \mid i \in I\right\}$. Ясно, что $\chi\left(G_{i}\right) \in S C_{b}^{l}(T, \mathscr{G})$. Следовательно, $\chi\left(F_{i}\right)=\mathbf{1}-\chi\left(T \backslash F_{i}\right) \in$ $S C^{b}(T, \mathscr{G})$. По лемме $1 \chi(A)=\sup \left\{\chi\left(G_{i} \cap F_{i}\right) \mid i \in I\right\} \in S C^{b}(T, \mathscr{G})$. Отсюда в силу леммы 1 следует, что $f \equiv \sum\left(x_{k} \chi\left(A_{k}\right) \mid k \in K\right) \in S C^{b}(T, \mathscr{G})$. 
TEOРема 1. Пусть $(T, \mathscr{G})$ - хаусдорфово пространство. Тогда $S(T, \mathscr{G})=$ $U^{f}(T, \mathscr{A}(T, \mathscr{G}))$.

ДокаЗАТЕЛЬСТво. Пусть $f \in S C_{b}^{l}(T, \mathscr{G})$ и $\varepsilon>0$. Разобьем интервал, содержаший множество значений функции $f$, точками $a_{k}$ так, что $a_{k+1}-a_{k} \leqslant \varepsilon / 3$. Рассмотрим множества

$$
\begin{aligned}
A_{k} & \equiv f^{-1}[] a_{k-1}, \infty[\backslash] a_{k+1}, \infty[] \\
& =f^{-1}[] a_{k-1}, \infty[] \backslash f^{-1}[] a_{k+1}, \infty[] \in \mathscr{A}(T, \mathscr{G})
\end{aligned}
$$

Так как $f^{-1}\left[\left[a_{k}, a_{k+1}\right]\right] \subset A_{k}$, то $\cup A_{k}=T$. Кроме того, $\omega\left(f, A_{k}\right) \leqslant a_{k+1}-$ $a_{k-1}<\varepsilon$. Значит, $f \in U^{f}(T, \mathscr{A}(T, \mathscr{G}))$. В силу предложения 1 этого пункта $S C^{b}(T, \mathscr{G}) \subset U^{f}(T, \mathscr{A}(T, \mathscr{G}))$.

Пусть даны функция $f \in F_{b}(T)$ и последовательность $\left(f_{n} \in U^{f}(T, \mathscr{A}(T, \mathscr{G})) \mid\right.$ $n \in N \subset \omega)$ такие, что $f=\lim \left(f_{n} \mid n \in N\right)$ в нормированном пространстве $\left(F_{b}(T),\|\cdot\|_{u}\right)$. Возьмем любое $\varepsilon>0$. По условию существует $n \in N$ такое, что $\left|f-f_{n}\right|<(\varepsilon / 3) \mathbf{1}$. Для $f_{n}$ сушествует конечное покрытие $\varkappa \equiv\left(A_{j} \in \mathscr{A}(T, \mathscr{G}) \mid\right.$ $j \in J)$ такое, что $\omega\left(f_{n}, A_{j}\right)<\varepsilon / 3$ для каждого $j \in J$. Тогда для каждых $s, t \in A_{j}$ вьполнено

$$
|f(s)-f(t)| \leqslant\left|f(s)-f_{n}(s)\right|+\left|f_{n}(s)-f_{n}(t)\right|+\left|f_{n}(t)-f(t)\right|<\varepsilon .
$$

Следовательно, $\omega\left(f, A_{j}\right)<\varepsilon$. Значит, $f \in U^{f}(T, \mathscr{A}(T, \mathscr{G}))$.

Из установленных свойств следует, что $S(T, \mathscr{G}) \subset U^{f}(T, \mathscr{A}(T, \mathscr{G}))$. Возьмем произвольную функцию $f \in U^{f}(T, \mathscr{A}(T, \mathscr{G}))$. В силу аксиомы выбора существует последовательность $\left(\varkappa_{n} \mid n \in \mathbb{N}\right)$ конечных покрытий $\varkappa_{n} \equiv\left(A_{n_{i}} \in \mathscr{A}(T, \mathscr{G}) \mid\right.$ $\left.i \in I_{n}\right)$ таких, что $\omega\left(f, A_{n_{i}}\right)<1 / n$. Разобьем интервал, содержаший множество значений функции $f$, точками некоторых последовательностей $\left(x_{n k} \mid k \in K_{n} \subset \omega\right)$ так, что $x_{n k+1}-x_{n k}=1 / n$. Рассмотрим множества $X_{n k} \equiv f^{-1}\left[\left[x_{n k}, x_{n k+1}\right]\right]$ и $B_{n k} \equiv \cup\left\{A_{n i} \mid A_{n i} \cap X_{n k} \neq \varnothing\right\} \in \mathscr{A}(T, \mathscr{G})$ и ступенчатые функции $f_{n} \equiv$ $\sup \left\{x_{n k} \chi\left(B_{n k}\right) \mid k \in K_{n}\right\} \in \operatorname{St}(T, \mathscr{A}(T, \mathscr{G}))$.

Пусть $t \in B_{n k} \cap B_{n l}$. Тогда $t \in A_{n i} \cap A_{n j}$ для некоторых $i$ и $j$ таких, что $A_{n i} \cap X_{n k} \neq \varnothing$ и $A_{n j} \cap X_{n l} \neq \varnothing$. Возьмем из последних двух множеств точки $r$ и $s$ соответственно. Тогда

$$
\begin{aligned}
x_{n l}-x_{n k} & \leqslant f(s)-x_{n k}<f(t)+1 / n-x_{n k}<f(r)+2 / n-x_{n k} \\
& \leqslant x_{n k+1}+2 / n-x_{n k}=3 / n .
\end{aligned}
$$

Это означает, что $x_{n k} \leqslant f_{n}(t)=\sup \left\{x_{n l} \mid t \in B_{n l}, l \in K_{n}\right\} \leqslant x_{n k}+3 / n$.

Пусть $t \in T$. Тогда $t \in X_{n k}$ для некоторого $k$ и $t \in A_{n i}$ для некоторого $i$. Следовательно, $t \in B_{n k}$. Поэтому $f(t)-f_{n}(t) \leqslant x_{n k+1}-x_{n k}=1 / n$ и $f_{n}(t)-$ $f(t) \leqslant x_{n k}+3 / n-x_{n k}=3 / n$ означает, что $\left|f-f_{n}\right| \leqslant(3 / n) \mathbf{1}$.

Возьмем $\varepsilon>0$ и подберем $n$ такое, что $3 / n<\varepsilon$. Тогда для каждого $p>n$ справедливо $\left|f-f_{p}\right| \leqslant(3 / p) \mathbf{1}<(3 / n) \mathbf{1}<\varepsilon \mathbf{1}$. Значит, $f=\lim \left(f_{n} \mid n \in \mathbb{N}\right)$ относительно $\|\cdot\|_{u}$. По лемме 2 этого пункта $f_{n} \in S C^{b}(T, \mathscr{G})$. Поэтому по определению $f \in S(T, \mathscr{G})$. В результате мы получаем необходимое равенство. 
СЛЕДСТВИЕ. $A \in \mathscr{A}(T, \mathscr{G})$ тогда и только тогда, когда $\chi(A) \in S(T, \mathscr{G})$.

Рассмотрим теперь на пространстве $(T, \mathscr{G})$ семейство $S_{c}(T, \mathscr{G})$ всех метаполунепрерывных функций с компактными носителями. Ясно, что $S_{c}(T, \mathscr{G})$ является усекаемым решеточным векторным подпространством в $S(T, \mathscr{G})$. Мы можем рассмотреть норму равномерной сходимости $\|\cdot\|_{u}$ на $S_{c}(T, \mathscr{G})$. Тогда $\left(S_{c}(T, \mathscr{G}),\|\cdot\|_{u}\right)-$ нормированное решеточное векторное пространство.

Пусть $\mu: \mathscr{M} \rightarrow \overline{\mathbb{R}}_{+}-$положительная широкая радоновская мера на $(T, \mathscr{G})$. Тогда $S_{c}(T, \mathscr{G}) \subset \mathrm{MI}(T, \mathscr{M}, \mu)$. Поэтому мы можем рассмотреть функционал $\varphi \equiv \Lambda(\mu) \mid S_{c}(T, \mathscr{G})$ (см. п. 4 из $\left.\S 1\right)$. Согласно п. $4 \S 1$ функционал $\varphi$ является поточечно $\sigma$-непрерывным. Но это свойство функционала $\varphi$ является очень общим. Нам нужно дополнительное свойство, связанное с компактной регулярностью меры $\mu$.

Подансамбль ансамбля $\mathscr{A}(T, \mathscr{G})$, состоящий из всех предкомпактных александровских множеств, обозначим через $\mathscr{A}_{c}(T, \mathscr{G})$. Ясно, что $A \in \mathscr{A}_{c}(T, \mathscr{G})$ тогда и только тогда, когда $\chi(A) \in S_{c}(T, \mathscr{G})$.

Лемма 3. Пусть $A \in \mathscr{A}(T, \mathscr{G})$. Тогда следующие условия равносильны:

1) $A \in \mathscr{A}_{c}(T, \mathscr{G})$;

2) существуют множество $B \in \mathscr{C}$ и конечные коллекции $\left(C_{i} \in \mathscr{C} \mid i \in I\right)$ u $\left(D_{i} \in \mathscr{C} \mid i \in I\right)$ такие, ито $A=\cup\left\{\left(B \cap C_{i}\right) \cap\left(B \backslash D_{i}\right) \mid i \in I\right\} ;$

3) существуют конечные коллекиии $\left(C_{i} \in \mathscr{C} \mid i \in I\right), \quad\left(D_{i} \in \mathscr{C} \mid i \in I\right)$ u $\left(E_{i} \in \mathscr{C} \mid i \in I\right)$ такие, ито $A=\cup\left\{C_{i} \cap\left(E_{i} \backslash D_{i}\right) \mid i \in I\right\}$.

ДокаЗАтельство. 1) $\Rightarrow 2$ ). Пусть $A=\cup\left\{F_{i} \cap G_{i} \mid i \in I\right\}$ для некоторых конечных коллекций $\left(F_{i} \in \mathscr{F} \mid i \in I\right)$ и $\left(G_{i} \in \mathscr{G} \mid i \in I\right)$ и $B \equiv \operatorname{cl} A \in \mathscr{C}$. Рассмотрим множества $H_{i} \equiv T \backslash G_{i} \in \mathscr{F}, C_{i} \equiv F_{i} \cap B \in \mathscr{C}$ и $D_{i} \equiv H_{i} \cap B \in \mathscr{C}$. Тогда $A=A \cap B=\cup\left\{\left(B \cap C_{i}\right) \cap\left(B \backslash D_{i}\right) \mid i \in I\right\}$.

$2) \Rightarrow 3)$. Эта импликация очевидна.

$3) \Rightarrow 1)$. Если $A=\cup\left\{C_{i} \cap\left(E_{i} \backslash D_{i}\right) \mid i \in I\right\}$, то $A \subset \cup\left\{C_{i} \cup E_{i} \mid i \in I\right\} \in \mathscr{C}$ влечет $A \in \mathscr{A}_{c}(T, \mathscr{G})$.

Функциональное семейство $A(T)$ на хаусдорфовом пространстве $(T, \mathscr{G})$ назовем топологически обиирным , если $S_{c}(T, \mathscr{G}) \subset A(T)$.

Положительный линейный функционал $\varphi$ на топологически обширном решеточном векторном пространстве $A(T)$ назовем положсительным внутренне комnактны.м, если для каждого $\varepsilon>0$ и для каждой убывающей последовательности $\left(A_{n} \in \mathscr{A}(T, \mathscr{G}) \mid n \in \omega\right)$ такой, что $\left\{\chi\left(A_{n} \backslash C\right) \mid n \in \omega, C \in \mathscr{C}\right\} \subset A(T)$, сушествуют $n \in \omega$ и $C \in \mathscr{C}$ такие, что $C \subset \cap\left\{A_{n} \mid n \in \omega\right\}$ и $\varphi\left(\chi\left(A_{n} \backslash C\right)\right)<\varepsilon$. Семейство всех положительных внутренне компактных линейных функционалов на $A(T)$ обозначим через $\left(A(T)^{z}\right)_{0}$.

Заметим, что если $A(T)=S_{c}(T, \mathscr{G})$, то условия $\left(A_{n} \in \mathscr{A}(T, \mathscr{G}) \mid n \in \omega\right)$ и $\left\{\chi\left(A_{n} \backslash C\right) \mid n \in \omega, C \in \mathscr{C}\right\} \subset A(T)$ равносильны условию $\left(A_{n} \in \mathscr{A}_{c}(T, \mathscr{G}) \mid\right.$ $n \in \omega)$. Если $A(T)=S(T, \mathscr{G})$, то условие $\left\{\chi\left(A_{n} \backslash C\right) \mid n \in \omega, C \in \mathscr{C}\right\}$ является излишним.

Линейный функционал $\varphi$ на $A(T)$ назовем бинарно-положительным внутренне компактным, если $\varphi=\varphi_{1}-\varphi_{2}$ для некоторых положительных внутренне компактных линейных функционалов $\varphi_{1}$ и $\varphi_{2}$ из $\left(A(T)^{z}\right)_{0}$. Семейство всех бинар- 
но-положительных внутренне компактных линейных функционалов на $A(T)$ обозначим через $A(T)^{z}$. Ясно, что $A(T)^{z} \subset A(T)^{\sim}$.

Следующая лемма дает внутреннюю характеризацию бинарно-положительных внутренне компактных линейных функционалов.

Лемма 4. Пусть $A(T)$ - топологически обиирное решеточное векторное пространство на $T$ и $\varphi \in A(T)^{\sim}$. Тогда следующие утверәсдения равносильны:

1)

2) для каждого $\varepsilon>0$ и каждой убьвающей последовательности $\left(A_{n} \in\right.$ $\mathscr{A}(T, \mathscr{G}) \mid n \in \omega)$ такой, что $\left\{\chi\left(A_{n} \backslash C\right) \mid n \in \omega, C \in \mathscr{C}\right\} \subset A(T)$, существуют $n \in \omega \quad u \quad C \in \mathscr{C}$ такие, что $C \subset \cap A_{n} \quad u \quad|\varphi f|<\varepsilon$ для каждой функиии $f \in A(T)+$ такой, что $f \leqslant \chi\left(A_{n} \backslash C\right)$.

Доказательство. 1$) \Rightarrow 2)$. Возьмем любые $\varepsilon>0$ и $\left(A_{n} \in \mathscr{A}(T, \mathscr{G}) \mid n \in \omega\right) \downarrow$ такие, что $\left\{\chi\left(A_{n} \backslash C\right) \mid n \in \omega, C \in \mathscr{C}\right\} \subset A(T)$. По условию $\varphi=\varphi_{1}-\varphi_{2}$ для некоторых положительных внутренне компактных линейных функционалов $\varphi_{1}$ и $\varphi_{2}$. Поэтому существуют $n_{i} \in \omega$ и $C_{i} \in \mathscr{C}$ такие, что $C_{i} \subset \cap A_{n}$ и $\varphi_{i}\left(\chi\left(A_{n_{i}} \backslash C_{i}\right)\right)<\varepsilon / 2$. Пусть $n \equiv \max \left\{n_{1}, n_{2}\right\}$ и $C \equiv C_{1} \cup C_{2}$.

Если $f \in A(T)_{+}$и $f \leqslant \chi\left(A_{n} \backslash C\right)$, то

$$
|\varphi f|=\left|\varphi_{1} f-\varphi_{2} f\right| \leqslant \varphi_{1} f+\varphi_{2} f \leqslant \varphi_{1} \chi\left(A_{n_{1}} \backslash C_{1}\right)+\varphi_{2} \chi\left(A_{n_{2}} \backslash C_{2}\right)<\varepsilon .
$$

$2) \Rightarrow 1)$. Возьмем любые $\varepsilon>0$ и $\left(A_{n} \in \mathscr{A}(T, \mathscr{G}) \mid n \in \omega\right) \downarrow$ такие, что $\left\{\chi\left(A_{n} \backslash C\right) \mid n \in \omega, C \in \mathscr{C}\right\} \subset A(T)$. По условию существуют $n \in \omega$ и $C \in \mathscr{C}$ такие, что $C \subset \cap A_{n}$ и $|\varphi f|<\varepsilon$ для каждой функции $f \in A(T)_{+}$такой, что $f \leqslant \chi\left(A_{n} \backslash C\right)$. По теореме Рисса-Канторовича

$$
\varphi_{+}\left(\chi\left(A_{n} \backslash C\right)\right)=\sup \left\{\varphi f \mid f \in A(T)_{+}, f \leqslant \chi\left(A_{n} \backslash C\right)\right\}<\varepsilon .
$$

Значит, функционал $\varphi_{+}$является положительным внутренне компактным. Аналогично, функционал $-\varphi$ - является положительным внутренне компактным. Так как $\varphi=\varphi_{+}-\left(-\varphi_{-}\right)$, то $\varphi$ является бинарно положительным внутренне компактным.

ПРЕДЛОЖЕНИЕ 3. Пусть $A(T)$ - топологически обиирное решеточное векторное пространство на хаусдорфовом пространстве $(T, \mathscr{G})$. Тогда $A(T)^{z}$ является l-идеалом в дедекиндово полном решеточно векторном пространстве $A(T)^{\sim} u\left(A(T)^{z}\right)_{+}=\left(A(T)^{z}\right)_{0}$.

ДокаЗАТЕльство. Обозначим первое семейство через $\Phi$ и второе через $\Psi$. Пусть $\varphi, \psi \in \Phi_{0}$ и $x, y \in \mathbb{R}_{+} \backslash\{0\}$. Ясно, что функционал $\eta \equiv x \varphi+y \psi$ является линейным и положительным. Возьмем любую последовательность $\left(A_{n} \in\right.$ $\mathscr{A}(T, \mathscr{G}) \mid n \in \omega) \downarrow$ такую, что $\left\{\chi\left(A_{n} \backslash C\right) \mid n \in \omega, C \in \mathscr{C}\right\} \subset A(T)$, и любое число $\varepsilon>0$. По условию сушествуют числа $l, m$ и компактные множества $C, D$ такие, что $C \cup D \subset \cap A_{n}, \varphi \chi\left(A_{l} \backslash C\right)<\varepsilon /(2 x)$ и $\varphi \chi\left(A_{m} \backslash D\right)<\varepsilon /(2 y)$. Следовательно, для $n \equiv \max \{l, m\}$ мы получаем

$$
\eta \chi\left(A_{n} \backslash C \cup D\right) \leqslant x \varphi \chi\left(A_{l} \backslash C\right)+y \psi \chi\left(A_{m} \backslash D\right)<\varepsilon .
$$

Это означает, что $\eta \in \Phi_{0}$. 
Из доказанного свойства следует, что $\Phi=\Phi_{0}-\Phi_{0}$ является векторным подпространством векторного пространства $\Psi$.

Пусть $\varphi \in \Phi_{0}, \psi \in \Psi$ и $0 \leqslant \psi \leqslant \varphi$. Тогда для $\left(A_{n} \in \mathscr{A}_{c}(T, \mathscr{G}) \mid n \in \omega\right) \downarrow$ такого, что $\left\{\chi\left(A_{n} \backslash C\right) \mid n \in \omega, C \in \mathscr{C}\right\} \subset A(T)$, и для $\varepsilon>0$ существуют $m$ и $C \subset \cap A_{n}$ такие, что $\varphi \chi\left(A_{m} \backslash C\right)<\varepsilon$. Следовательно, $\psi \chi\left(A_{m} \backslash C\right)<\varepsilon$ означает, что $\psi \in \Phi_{0}$.

Пусть теперь $\varphi \in \Phi, \psi \in \Psi$ и $|\psi| \leqslant \varphi$. По определению $\varphi=\varphi_{1}-\varphi_{2}$ для некоторых $\varphi_{1}, \varphi_{2} \in \Phi_{0}$. Поэтому по свойству, доказанному выше, условия $0 \leqslant \psi_{+} \leqslant|\psi| \leqslant \varphi_{1}+\varphi_{2} \in \Phi_{0}$ влекут $\psi_{+} \in \Phi_{0}$. Аналогично, $-\psi_{-} \in \Phi_{0}$. Значит, $\psi=\psi_{+}-\left(-\psi_{-}\right) \in \Phi$. Следовательно, $\Phi$ является $l$-идеалом в $\Psi$.

Наконец, пусть $\varphi \in \Phi_{+}$. По определению $\varphi=\varphi_{1}-\varphi_{2}$ для некоторых $\varphi_{1}, \varphi_{2} \in \Phi_{0}$. Поэтому $0 \leqslant \varphi \leqslant \varphi_{1}$ в $\Psi$. По доказанному выше $\varphi \in \Phi_{0}$. Это означает, что $\Phi_{+}=\Phi_{0}$.

Из равенства $\left(A(T)^{z}\right)_{+}=\left(A(T)^{z}\right)_{0}$ следует, что мы можем опускать слова "бинарно-положительный" и называть бинарно-положительные внутренне компактные линейные функционалы просто внутренне компактными линейными функиионалами.

Линейный функционал $\varphi$ на функциональном семействе $A(T)$ назовем локально ограниченны.м, если для каждой точки $t \in T$ существует открытая окрестность $G$ точки $t$ такая, что $\sup \{|\varphi f| \mid(f \in A(T)) \wedge(|f| \leqslant \chi(G))\}<\infty$.

ЛЕмма 5. Пусть $A(T)$ - решеточное векторное пространство и $\varphi \in A(T)^{\sim}$. Тогда $\varphi$ является локально ограниченным, если и только если $|\varphi|$ является локально ограниченным.

ДокАЗАТЕЛЬСТво. Пусть $\varphi$ является локально ограниченным и $t \in T$. Тогда $x \equiv \sup \{|\varphi f||f \in A(T) \wedge| f \mid \leqslant \chi(G)\}<\infty$ для некоторой открытой окрестности $G$ точки $t$. По теореме Рисса-Канторовича $|\varphi||f|=\sup \{|\varphi g| \mid g \in A(T) \wedge$ $|g| \leqslant|f|\}$. Поэтому $f, g \in A(T)$ и $|g| \leqslant|f| \leqslant \chi(G)$ влекут $|\varphi g| \leqslant x$ и $|\varphi||f| \leqslant x$. В результате ||$\varphi|f| \leqslant|\varphi||f| \leqslant x$. Это означает, что $|\varphi|$ локально ограничен.

Пусть теперь $|\varphi|$ локально ограничен и $t \in T$. Тогда $y \equiv \sup \{\| \varphi|f| \mid f \in A(T) \wedge$ $|f| \leqslant \chi(H)\}<\infty$ для некоторой открытой окрестности $H$ точки $t$. Если $f \in A(T)$ и $|f| \leqslant \chi(H)$, то $g \equiv|f| \in A(T)$ и $|g| \leqslant \chi(H)$. Откуда $|\varphi f| \leqslant|| \varphi|g| \leqslant y$. Это означает, что $\varphi$ локально ограничен.

Линейный функционал на топологически обширном решеточном векторном пространстве $A(T)$ назовем тонким, если он является поточечно $\sigma$-непрерывньм, внутренне компактным и локально ограниченным. Семейство всех тонких линейных функционалов на $A(T)$ обозначим через $A(T)^{\triangle}$. Если $A(T) \subset F_{b}(T)$, то семейство $A(T)^{\triangle} \cap\left(A(T),\|\cdot\|_{u}\right)^{\prime}$ обозначим через $A(T)^{\ominus}$.

ПРЕДЛОЖЕНИЕ 4. Пусть $(T, \mathscr{G})$ - хаусдорфово пространство и $A(T)-$ топологически обширное решеточное векторное пространство на T. Тогда:

1) $A(T)^{\triangle}$ является l-идеалом в дедекиндово полном решеточном векторном пространстве $A(T)^{\sim}$;

2) $\varphi \in\left(A(T)^{\triangle}\right)_{+}\left[\left(A(T)^{\otimes}\right)_{+}\right]$, если и только если $\varphi$ является положительным внутренне компактным, поточечно б-непрерывным и локально ограниченным (равномерно ограниченным) линейным функиионалом. 
ДокаЗАТЕЛЬСТво. 1) Пусть $\varphi \in A(T)^{\triangle}, \psi \in A(T)^{\sim}$ и $|\psi| \leqslant|\varphi|$. По теореме из п. $2 \S 1 \psi \in A(T)^{\wedge}$. По предложению 3 этого пункта $\psi \in A(T)^{z}$. По лемме $5|\varphi|$ локально ограничен. Поэтому для каждой точки $t \in T$ сушествует открытая окрестность $G$ точки $t$ такая, что $x \equiv \sup \{|| \varphi|f||f \in A(T) \wedge| f \mid \leqslant \chi(G)\}<\infty$. Следовательно, $f \in A(T)$ и $|f| \leqslant \chi(G)$ влекут $|\psi f| \leqslant|\psi||f| \leqslant|\varphi||f| \leqslant x$, поскольку $|f| \in A(T)$. Это означает, что $\psi$ является локально ограниченным. В результате $\psi \in A(T)^{\triangle}$.

2) Пусть $\varphi \in\left(A(T)^{\triangle}\right)_{+}$. Тогда $\varphi \in\left(A(T)^{\wedge}\right)_{+} \cap\left(A(T)^{z}\right)_{+}$. По предложению $3 \varphi$ является положительным внутренне компактным.

СлеДСТвИЕ 1. В условиях предложения $4 \quad A(T)^{\triangle}$ является дедекиндово полным решеточным векторным пространством; наименьшие верхние (наибольшие нижсие) границь множеств любой допустимой мощности в упорядоченных множествах $A(T)^{\sim}$ и $A(T)^{\triangle}$ совпадают.

СлЕДСТВИЕ 2. В условиях предложения 4 следующие условия равносильны:

1) $\varphi \in A(T)^{\triangle}\left[\varphi \in A(T)^{\otimes}\right]$;

2) $\varphi=\varphi_{1}-\varphi_{2}$ для некоторых положительных внутренне компактных, поточечно б-непрерывных и локально ограниченных (равномерно ограниченных) линейных функционалов $\varphi_{1} u \varphi_{2}$.

СлЕДСТВИЕ 3. В условиях предложения $4 A(T) \otimes$ является l-идеалом в дедекиндово полном решеточном векторном пространстве $A(T)^{\triangle}$. Cле-


пространством.

ПРЕДЛОЖЕНИЕ 5. Пусть $(T, \mathscr{G}, \mathscr{M}, \mu)$ - Хаусдорфово пространство с положительной широкой радоновской мерой $\mu: \mathscr{M} \rightarrow \overline{\mathbb{R}}_{+} u A(T)$ - решеточное векторное пространство на $T$ такое, что $S_{c}(T, \mathscr{G}) \subset A(T) \subset \operatorname{MI}(T, \mathscr{M}, \mu)$. Тогда положстельный функиионал $\varphi \equiv \Lambda(\mu) \mid A(T)$ является тонким (см. n. $4 \S 1)$. Если мера $\mu$ ограничена, то $\varphi \in A(T) \oslash$.

ДокАЗАТЕльство. Возьмем последовательность $\left(A_{n} \in \mathscr{A}(T, \mathscr{G}) \mid n \in \omega\right) \downarrow$ такую, что $\left\{\chi\left(A_{n} \backslash C\right) \mid n \in \omega, C \in \mathscr{C}\right\} \subset A(T)$. Тогда $\left\{A_{n} \mid n \in \omega\right\} \subset \mathscr{M}^{f}(\mu)$. Рассмотрим множество $K \equiv \cap A_{n} \in \mathscr{M}^{f}(\mu)$. Так как мера $\mu$ компактно регулярна, для каждого $\varepsilon>0$ существует компактное множество $C$ такое, что $C \subset K$ и $\mu K-\varepsilon / 2<\mu C$. По свойству меры $\mu K=\lim \left(\mu A_{n} \mid n \in \omega\right)$. Поэтому для $\varepsilon$ существует $n$ такое, что $\mu A_{n}-\mu K<\varepsilon / 2$. В результате

$$
\mu\left(A_{n} \backslash C\right)=\mu A_{n}-\mu C=\left(\mu A_{n}-\mu K\right)+(\mu K-\mu C)<\varepsilon .
$$

Следовательно, $\varphi \chi\left(A_{n} \backslash C\right)=\mu\left(A_{n} \backslash C\right)<\varepsilon$. Таким образом, $\varphi$ является внутренне компактным.

Согласно п. $4 \S 1 \varphi$ является поточечно $\sigma$-непрерывным. Далее, для каждой точки $t$ сушествует открытая окрестность $G$ такая, что $\mu G=\sup \{\mu M \mid M \in$ $\mathscr{M} \wedge M \subset G\}<\infty$. Пусть $f \in A(T) \subset \operatorname{MI}(T, \mathscr{M}, \mu)$ и $|f| \leqslant \chi(G)$. Тогда $|\varphi f| \leqslant$ $\int|f| d \mu \leqslant \int \chi(G) d \mu=\mu G$. Это означает, что $\varphi$ является локально ограниченным. Если $\mu$ ограничено, то $\varphi$ равномерно ограничено. 
5. Теоремы об общем радоновском представлении. В этом пункте мы дадим одно из возможных решений проблемы обшего радоновского представления. Будут представлены биективная и изоморфная версии этого решения.

Функциональное семейство $A(T)$ на пространстве $(T, \mathscr{G})$ назовем $\mathscr{F}$ - усекаемы. если $f \wedge \chi(F) \in A(T)$ для каждых $f \in A(T)$ и $F \in \mathscr{F}$. Очевидно, что семейства $S_{c}(T, \mathscr{G})$ и $S(T, \mathscr{G})$ являются $\mathscr{F}$-усекаемыми.

Рассмотрим отображение $V^{\wedge}:\left(S_{c}(T, \mathscr{G})^{\wedge}\right)_{+} \rightarrow \mathfrak{M}(T)_{0}$ из п. $4 \S 1$ и его ограничения $V^{\#} \equiv V^{\wedge} \mid\left(S_{c}(T, \mathscr{G})^{\triangle}\right)_{+}$и $V_{b}^{\#} \equiv V^{\#} \mid\left(S_{c}(T, \mathscr{G})^{\ominus}\right)_{+}$.

ПРЕДЛОЖЕНИЕ 1. Пусть $(T, \mathscr{G})$ - хаусдорфово пространство, и пусть $\varphi \in$ $\left(S_{c}(T, \mathscr{G})^{\triangle}\right)_{+} u \mu \equiv V^{\#} \varphi: \mathscr{M} \rightarrow \overline{\mathbb{R}}_{+}$. Тогда:

1) $\mathscr{J}_{\sigma}\left(S_{c}(T, \mathscr{G})\right) \subset \mathscr{A}_{c}(T, \mathscr{G})_{\delta}$

2) $\mu \in \mathfrak{R M}^{w e}(T, \mathscr{G})_{0}$;

3) $\varphi$ представляется интегралом Лебега над измеримым пространством $(T, \mathscr{M}, \mu)$;

4) мера $\mu$ является единственной в следующем смысле: если существует другая мера $\nu: \mathcal{N} \rightarrow \overline{\mathbb{R}}_{+}$из $\mathfrak{R M}^{w e}(T, \mathscr{G})_{0}$ такая, что $\varphi$ представляется интегралом Лебега над пространством $(T, \mathcal{N}, \nu)$, то $\mathcal{N}=\mathscr{M} u \nu=\mu$;

$5)$ если $\varphi \in\left(S_{c}(T, \mathscr{G})^{\ominus}\right)_{+}$, то мера $\mu$ ограничена.

ДоказАТЕЛЬСтво. Обозначим $S_{c}(T, \mathscr{G})$ через $A(T)$. Рассмотрим ансамбль $\mathscr{K} \equiv \mathscr{J}_{\sigma}(A(T))$. По теоремам из пп. $4,3 \S 1 \mu$ является положительной, широкой, полной, сильно насьшенной, $\mathscr{K}$-насьшенной, внутренне $\mathscr{K}$-регулярной и верхне $\mathscr{K}$ - $\sigma$-непрерывной мерой такой, что $\mathscr{K} \subset \mathscr{M}^{f}(\mu)$, и $\varphi$ представляется интегралом Лебега над пространством $(T, \mathscr{M}, \mu)$. Кроме того, если $\varphi$ равномерно ограничено, то $\mu$ ограничено.

Более того, $\mu M=\sup \{\mu K \mid K \in \mathscr{K} \wedge K \subset M\}$ и $\mu K \equiv \inf \left\{\varphi f \mid f \in A(T)_{+} \wedge\right.$ $f \geqslant \chi(K)\}$.

Так как $\chi(C) \in A(T)$ для каждого компактного множества $C$, мы заключаем, что $\mathscr{C} \subset \mathscr{K}$.

Пусть $F \in \mathscr{F}$ и $K \in \mathscr{K}$. Тогда $\left(f_{n} \in A(T)_{+} \mid n \in \omega\right) \downarrow \chi(K)$ для некоторой последовательности $\left(f_{n}\right)$. Так как $A(T)$ является $\mathscr{F}$-усекаемым, мы можем взять функции $g_{n} \equiv f_{n} \wedge \chi(F) \in A(T)_{+}$. Из $\left(g_{n} \mid n \in \omega\right) \downarrow \chi(K \cap F)$ заключаем, что $K \cap F \in \mathscr{K} \subset \mathscr{M}$. Так как мера $\mu$ является $\mathscr{K}$-насышенной, получаем $F \in \mathscr{M}$. Таким образом, $\mathscr{F} \subset \mathscr{M}$. Следовательно, $\mathscr{G} \subset \mathscr{M}$.

Пусть $K \in \mathscr{K}$ и $\left(f_{n} \in A(T)_{+} \mid n \in \omega\right) \downarrow \chi(K)$. Рассмотрим множества $S f_{n} \equiv\left\{t \in T \mid f_{n}(t) \geqslant 1\right\}$. Тогда $\left(S f_{n} \mid n \in \omega\right) \downarrow K$ в $\mathscr{P}(T)$. Так как $f_{n}$ является метаполунепрерывной, существует конечное покрытие $\left(B_{n j} \in \mathscr{A}(T, \mathscr{G}) \mid j \in J_{n}\right)$ множества $T$ такое, что $\omega\left(f_{n}, B_{n j}\right)<1 /(n+1)$. Рассмотрим множества $J_{n}^{\prime} \equiv\{j \in$ $\left.J_{n} \mid B_{n j} \cap S f_{n} \neq \varnothing\right\}$ и $B_{n} \equiv \cup\left\{B_{n j} \mid j \in J_{n}^{\prime}\right\} \in \mathscr{A}(T, \mathscr{G})$. Если $t \in B_{n}$, то $t \in B_{n j}$ для некоторого $j \in J_{n}^{\prime}$. Возьмем некоторую точку $s \in B_{n j} \cap S f_{n}$. Тогда $\left|f_{n}(t)-f_{n}(s)\right|<1 /(n+1)$ влечет $f_{n}(t)>f_{n}(s)-1 /(n+1) \geqslant n /(n+1)$. Поэтому $S f_{n} \subset B_{n} \subset S_{n} \equiv\left\{t \in T \mid f_{n}(t)>n /(n+1)\right\} \subset \operatorname{supp} f_{n} \in \mathscr{C}$.

Если $t \notin K$, то $f_{n}(t)<1 / 2$ для некоторого $n \geqslant 1$. Следовательно, $t \notin S_{n}$, откуда $\cap S_{n} \subset K$. В результате $K=\cap\left\{S f_{n} \mid n \in \omega\right\} \subset \cap B_{n} \subset \cap S_{n} \subset K$ влечет $K=\cap B_{n} \in \mathscr{A}_{c}(T, \mathscr{G})_{\delta}$. 
Возьмем множества $A_{n} \equiv \cap\left\{B_{m} \mid m \in n+1\right\} \in \mathscr{A}_{c}(T, \mathscr{G})$. Тогда $\left(A_{n} \mid n \in \omega\right) \downarrow K$ и $\left\{\chi\left(A_{n} \backslash C\right) \mid n \in \omega, C \in \mathscr{C}\right\} \subset A(T)$. Так как $\varphi$ является внутренне компактным, для каждого $\varepsilon>0$ сушествуют $n \in \omega$ и $C \in \mathscr{C}$ такие, что $C \subset K$ и $\varphi \chi\left(A_{n} \backslash C\right)<\varepsilon$. Тогда $\mu K-\mu C \leqslant \mu A_{n}-\mu C=\mu\left(A_{n} \backslash C\right)=\int \chi\left(A_{n} \backslash C\right) d \mu=\varphi \chi\left(A_{n} \backslash C\right)<\varepsilon$. Поэтому $\mu K=\sup \{\mu C \mid C \in \mathscr{C} \wedge C \subset K\}$.

Пусть $M \in \mathscr{M}$ и возьмем число $x$ такое, что $x<\mu M$. Тогда сушествует $K \in \mathscr{K}$ такое, что $K \subset M$ и $x<\mu K$. Далее, существует $C \in \mathscr{C}$ такое, что $C \subset K$ и $x<\mu C$. Таким образом, $x \leqslant \sup \{\mu C \mid C \in \mathscr{C} \wedge C \subset M\} \leqslant \mu M$. Так как $x$ произвольно, мы заключаем, что $\mu M=\sup \{\mu C \mid C \in \mathscr{C} \wedge C \subset M\}$. Это означает, что $\mu$ является компактно регулярным.

Далее, для каждой точки $t \in T$ существует открытая окрестность $G$ такая, что $x \equiv \sup \{|\varphi f||f \in A(T) \wedge| f \mid \leqslant \chi(G)\}<\infty$. Как доказано вьше, $\mu G=\sup \{\mu C \mid C \in \mathscr{C} \wedge C \subset G\}$. Поэтому $\mu G=\sup \{\varphi \chi(C) \mid C \in \mathscr{C} \wedge$ $\chi(C) \leqslant \chi(G)\} \leqslant x<\infty$. Таким образом, мера $\mu$ локально ограничена. В результате $\mu$ является широкой положительной радоновской мерой.

Наконец, предположим, что $\varphi$ является равномерно ограниченным. Тогда $\mu T=\sup \{\varphi \chi(C) \mid C \in \mathscr{C}\} \leqslant \sup \{\varphi f \mid f \in A(T) \wedge 0 \leqslant f \leqslant \mathbf{1}\}<\infty$, т.е. $\mu$ ограничено.

Теперь нам нужно проверить единственность $\mu$. Пусть $\nu: \mathscr{N} \rightarrow \overline{\mathbb{R}}_{+}-$мера из утверждения 4). Возьмем любое $C \in \mathscr{C}$. Тогда $\chi(C) \in A(T)$ влечет равенство $\mu C=\int \chi(C) d \mu=\varphi \chi(C)=\int \chi(C) d \nu=\nu C$.

Пусть $N \in \mathscr{N}$ и $C \in \mathscr{C}$. По свойству компактной регулярности $\nu$ мы можем найти некоторые последовательности $\left(K_{n} \subset C \cap N \mid n \in \mathbb{N}\right) \uparrow$ и $\left(L_{n} \subset C \backslash N \mid\right.$ $n \in \mathbb{N}) \uparrow$ компактных множеств такие, что $\nu(C \cap N)=\sup \left\{\nu K_{n} \mid n \in \mathbb{N}\right\}$ и $\nu(C \backslash N)=\sup \left\{\nu L_{n} \mid n \in \mathbb{N}\right\}$. Рассмотрим дизъюнктные множества $K \equiv \cup K_{n} \subset$ $C \cap N$ и $L \equiv \cup L_{n} \subset C \backslash N$ из $\mathscr{M}$.

Мы имеем $\nu\left(\left(C \backslash K_{m}\right) \backslash L_{n}\right)=\nu\left(C \backslash K_{m}\right)-\nu\left(\left(C \backslash K_{m}\right) \cap L_{n}\right)=\nu C-\nu\left(C \cap K_{m}\right)-$ $\nu L_{n}=\nu C-\nu K_{m}-\nu L_{n}$. Следовательно, $\mu K=\sup \mu K_{n}=\sup \nu K_{n}=\nu(C \cap N)$ и $\mu L=\sup \mu L_{n}=\sup \nu L_{n}=\nu(C \backslash N)$. Поэтому $\mu(K \cup L)=\mu K+\mu L=\nu(C \cap N)+$ $\nu(C \backslash N)=\nu C$. Значит, $\nu C=\mu C=\mu(C \backslash(K \cup L))+\mu(K \cup L)=\mu(C \backslash(K \cup L))+\nu C$, откуда $\mu(C \backslash(K \cup L))=0$.

Из $(C \cap N) \backslash K \subset C \backslash(K \cup L)$ в силу полноты $\mu$ мы заключаем, что $(C \cap N) \backslash$ $K \in \mathscr{M}$. Значит, $N \cap C=((C \cap N) \backslash K) \cup K \in \mathscr{M}$ для любого $C \in \mathscr{C}$.

Возьмем любое множество $M \in \mathscr{M}^{f}(\mu)$. Так как $\mu$ компактно регулярно, мы имеем $\mu M=\sup \{\mu D \mid D \in \mathscr{C} \wedge D \subset M\}$. Поэтому существует последовательность $\left(D_{n} \in \mathscr{C} \mid n \in \mathbb{N}\right) \uparrow$ такая, что $\cup\left\{D_{n} \mid n \in \mathbb{N}\right\} \subset M$ и $\mu M=\sup \left\{\mu D_{n} \mid n \in \mathbb{N}\right\}$. Рассмотрим множества $E \equiv \cup\left\{D_{n} \mid n \in \mathbb{N}\right\} \quad$ и $H \equiv M \backslash E$ из $\mathscr{M}$. Тогда $\mu D_{n} \leqslant \mu E=\mu M-\mu H$ для каждого $n$ влечет $\mu M \leqslant \mu M-\mu H$. Значит, $\mu H=0$. Из $(N \cap M) \backslash E \subset H$ в силу полноты $\mu$ мы заключаем, что $(N \cap M) \backslash E \in \mathscr{M}$. С другой стороны, по доказанному вьше $(N \cap M) \cap E=\cup\left\{\left(N \cap D_{n}\right) \cap M \mid n \in \mathbb{N}\right\} \in \mathscr{M}$. В результате $N \cap M \in \mathscr{M}$. Из сильной насьшенности $\mu$ теперь следует, что $N \in \mathscr{M}$. Таким образом, $\mathscr{N} \subset \mathscr{M}$. Точно таким же образом $\mathscr{M} \subset \mathscr{N}$.

Теперь из свойства компактной регулярности следует, что $\mu=\nu$.

СлеДСТвИЕ. $\operatorname{rng} V^{\#} \subset \mathfrak{R M}^{w e}(T, \mathscr{G})_{0} u \operatorname{rng} V_{b}^{\#} \subset \mathfrak{R M}_{b}^{w e}(T, \mathscr{G})_{+}$.

Если $\varphi \in\left(S_{c}(T, \mathscr{G})^{\triangle}\right)_{+}$и $\mu \equiv V^{\#} \varphi: \mathscr{M} \rightarrow \overline{\mathbb{R}}_{+}$, то по предложению 1 этого пункта $\mathscr{K} \equiv \mathscr{J}_{\sigma}\left(S_{c}(T, \mathscr{G})\right) \subset \mathscr{A}_{c}(T, \mathscr{G})_{\delta} \subset \mathscr{B}(T, \mathscr{G})$. Следовательно, $\mathscr{B}(T, \mathscr{K}) \subset$ 
$\mathscr{B}(T, \mathscr{G}) \subset \mathscr{M} . \quad$ В результате $\mathscr{B}(T, \mathscr{K}) \subset \mathscr{B}(T, \mathscr{G}) \subset \cap\left\{\operatorname{dom} V^{\#} \varphi \mid \varphi \in\right.$ $\left.\left(S_{c}(T, \mathscr{G})^{\triangle}\right)_{+}\right\}$. Это означает согласно п. $4 \S 1$, что мы можем взять $\mathscr{M}_{0} \equiv$ $\mathscr{B}(T, \mathscr{G})$ и можем рассмотреть отображения $V:\left(S_{c}(T, \mathscr{G})^{\triangle}\right)_{+} \rightarrow \operatorname{Meas}\left(T, \mathscr{M}_{0}\right)$ и $V_{b}:\left(S_{c}(T, \mathscr{G})^{\ominus}\right)_{+} \rightarrow \operatorname{Meas}_{b}\left(T, \mathscr{M}_{0}\right)$ из п. $4 \S 1$.

ПРЕДЛОЖЕНИЕ 2. Пусть $(T, \mathscr{G})-$ хаусдорфово пространство, $\mu \equiv V^{\#} \varphi$, $\mu_{0} \equiv V \varphi: \mathscr{M}_{0} \rightarrow \overline{\mathbb{R}}_{+} u \varphi \in\left(S_{c}(T, \mathscr{G})^{\triangle}\right)_{+}$. Tогдa:

1) $\mu_{0} \in \mathfrak{R M}^{w o}(T, \mathscr{G})_{0}, \mu_{0}=\mu \mid \mathscr{M}_{0} u \mu=\left(\mu_{0}\right)^{\#}$;

2) ч представляется интегралом Лебега над измеримым пространством $\left(T, \mathscr{M}_{0}, \mu_{0}\right)$

3) мера $\mu_{0}$ является единственной в следующем смысле: если существует другая положительная широкая борелевско-радоновская мера $\varkappa: \mathscr{M}_{0} \rightarrow \overline{\mathbb{R}}_{+}$ такая, что ч представляется интегралом Лебега над пространством $\left(T, \mathscr{M}_{0}, \varkappa\right)$, mo $\varkappa=\mu_{0}$

4) если $\varphi \in\left(S_{c}(T, \mathscr{G})^{\ominus}\right)_{+}$, то мера $\mu$ ограничена.

ДокАЗАТЕЛЬСТво. Согласно п. $4 \S 1 \mu_{0}=\mu \mid \mathscr{M}_{0}$. По предложению 1 этого пункта $\mu$ является полной сильно насьщенной радоновской мерой. Поэтому по лемме из п. $5 \S 1 \mu_{0}$ является борелевско-радоновской мерой и $\mu=\left(\mu_{0}\right)^{\#}$. Утверждения 2) и 4) следуют из теоремы из п. $4 \S 1$.

Используя точно такие же рассуждения, как и в доказательстве предложения 1 , мы выводим, что $\mu_{0}$ и $\varkappa$ совпадают на $\mathscr{C}$. Теперь из свойства компактной регулярности следует, что $\mu_{0}=\varkappa$.

СлЕДСТВИЕ. $\operatorname{rng} V \subset \mathfrak{R M}^{w o}(T, \mathscr{G})_{0} u \operatorname{rng} V_{b} \subset \mathfrak{R M}_{b}^{w o}(T, \mathscr{G})_{+}$.

Tеорема 1. Пусть $(T, \mathscr{G})$ - хаусдорфово пространство. Пусть $V^{\#} u V-$ отображения из предложений 1 и 2. Тогда:

1) $V^{\#}$ является биекцией из множества $\left(S_{c}(T, \mathscr{G})^{\triangle}\right)_{+}$на множество $\mathfrak{R M}^{w e}(T, \mathscr{G})_{0}$;

2) $V$ является биекцией из множества $\left(S_{c}(T, \mathscr{G})^{\triangle}\right)_{+}$на множество $\mathfrak{R M}^{w o}(T, \mathscr{G})_{0}$

3) $V_{b}^{\#}$ является биекцией из множества $\left(S_{c}(T, \mathscr{G})^{\oplus}\right)+$ на множество $\mathfrak{R M}_{b}^{w e}(T, \mathscr{G})_{+}$;

4) $V_{b}$ является биекцией из множества $\left(S_{c}(T, \mathscr{G})^{\oplus}\right)_{+}$на множество $\mathfrak{R M}_{b}^{w o}(T, \mathscr{G})_{+}$;

5) если $\varphi, \psi \in\left(S_{c}(T, \mathscr{G})^{\triangle}\right)_{+} u x, y \in \mathbb{R}_{+}, \operatorname{mo} V(x \varphi+y \psi)=x V \varphi+y V \psi$;

6) $е с л и \varphi, \psi \in\left(S_{c}(T, \mathscr{G})^{\ominus}\right)_{+} u x, y \in \mathbb{R}_{+}, \operatorname{mo} V_{b}(x \varphi+y \psi)=x V_{b} \varphi+y V_{b} \psi$;

7) отображения $V$ и $V_{b}$ изотонны и сохраняют любье порядковые границы.

ДОКАЗАТЕЛЬСТВО. По следствиям из предложений 1 и 2 этого пункта $\operatorname{rng} V^{\#} \subset \mathfrak{R M}^{w e}(T, \mathscr{G})_{0}$ и $\operatorname{rng} V \subset \mathfrak{R M}^{\text {wo }}(T, \mathscr{G})_{0}$. Согласно п. $4 \S 1 V^{\#}$ и $V$ инъективны. Поэтому нам нужно проверить только, что $V^{\#}$ и $V$ сюръективны.

Пусть $\mu: \mathscr{M} \rightarrow \overline{\mathbb{R}}_{+}$принадлежит $\mathfrak{R} \mathfrak{M}^{w e}(T, \mathscr{G})_{0}$. Тогда $S_{c}(T, \mathscr{G}) \subset \operatorname{MI}(T, \mathscr{M}, \mu)$. По предложению 5 из п. $4 \S 2$ положительньй функционал $\varphi \equiv \Lambda(\mu) \mid S_{c}(T, \mathscr{G})$ является тонким. По определению $\varphi f=\int f d \mu$ для каждого $f \in S_{c}(T, \mathscr{G})$. Следовательно, в силу утверждения 4) предложения $1 \mu=V^{\#} \varphi$. Таким образом, $V^{\#}$ сюръективно. 
Если $\mu \in \mathfrak{R M}^{\text {wo }}(T, \mathscr{G})_{0}$, то по тем же причинам, как и выше, в силу утверждения 3) предложения 2 этого пункта $\mu=V \varphi$. Таким образом, $V$ сюръективно.

По следствиям к предложениям 1 и $2 \operatorname{rng} V_{b}^{\#} \subset \mathfrak{R M}_{b}^{w e}(T, \mathscr{G})_{+}$и $\operatorname{rng} V_{b} \subset$ $\mathfrak{R M}_{b}^{w o}(T, \mathscr{G})_{+} \cdot \quad$ Если $\mu \in \mathfrak{R M}_{b}^{w e}(T, \mathscr{G})_{+}$, то $\varphi \in\left(S_{c}(T, \mathscr{G})^{\ominus}\right)_{+} \cdot$ Поэтому отображения $V_{b}^{\#}$ и $V_{b}$ сюръективны.

Пусть теперь $\mu=V \varphi$ и $\nu=V \psi$. По предложению из п. $5 \S 1 \lambda \equiv x \mu+$ $y \nu \in \mathfrak{R M}^{w o}(T, \mathscr{G})_{0}$. По предложению $2 S_{c}(T, \mathscr{G}) \subset \operatorname{MI}\left(T, \mathscr{M}_{0}, \mu\right) \cap \operatorname{MI}\left(T, \mathscr{M}_{0}, \nu\right)$. Однако $\mathrm{MI}\left(T, \mathscr{M}_{0}, \lambda\right)=\operatorname{MI}\left(T, \mathscr{M}_{0}, \mu\right) \cap \operatorname{MI}\left(T, \mathscr{M}_{0}, \nu\right)$ и $\int f d \lambda=x \int f d \mu+y \int f d \nu$ для каждой функции $f \in \operatorname{MI}\left(T, \mathscr{M}_{0}, \lambda\right)$. Поэтому $S_{c}(T, \mathscr{G}) \subset \operatorname{MI}\left(T, \mathscr{M}_{0}, \lambda\right)$ и для каждой функции $f \in S_{c}(T, \mathscr{G})$ мы имеем по предложению $2(x \varphi+y \psi) f=$ $x \int f d \mu+y \int f d \nu=\int f d \lambda$. В силу утверждения 3$)$ предложения $2 \lambda=V(x \varphi+y \psi)$. В качестве следствия мы получаем такое же свойство для $V_{b}$.

Пусть $\varphi \leqslant \psi$. Если $C$ - компактное множество, то $\chi(C) \in S_{c}(T, \mathscr{G})_{+}$. Поэтому в силу утверждения 2) предложения $2 \mu C=\int \chi(C) d \mu=\varphi \chi(C) \leqslant \psi \chi(C)=$ $\int \chi(C) d \nu=\nu C$. Теперь из компактной регулярности этих мер мы выводим, что $\mu \leqslant \nu$.

Обратно, пусть $\mu \leqslant \nu$. Тогда $\varphi f=\int f d \mu \leqslant \int f d \nu=\psi f$ для каждой функции $f \in S_{c}(T, \mathscr{G})_{+}$. Это означает, что $\varphi \leqslant \psi$. Таким образом, $V$ является изотонным. Следовательно, $V$ сохраняет любые порядковые границы. Для $V_{b}$ рассуждения такие же.

Теперь наша цель - расширить отображения $V$ и $V_{b}$ на все множества $S_{c}(T, \mathscr{G})^{\triangle}$ и $S_{c}(T, \mathscr{G})^{\ominus}$ соответственно. Для расширения отображения $V_{b}$ мы имеем такую же процедуру, как и в доказательстве второй теоремы Рисса-Радона о представлении из п. $6 \oint 1$. Однако расширение отображения $V$ является трудной задачей, для решения которой и были изобретены радоновские бимеры.

Рассмотрим композиционное отображение $E \circ V:\left(S_{c}(T, \mathscr{G})^{\triangle}\right)_{+} \rightarrow \mathfrak{R B}(T, \mathscr{G})_{+}$.

TЕОрема 2. Пусть $(T, \mathscr{G})$ - хаусдорфово пространство. Тогда:

1) отображение $E \circ V$ имеет единственное расиирение до изоморфизма $\mathfrak{I}: S_{c}(T, \mathscr{G})^{\triangle} \longmapsto \mathfrak{R B}(T, \mathscr{G})$ между данными решеточными векторными пространствами такого, что $\mathfrak{I} \varphi=E\left(V\left(\varphi_{+}\right)\right)-E\left(V\left(-\varphi_{-}\right)\right)$для каждого $\varphi \in S_{c}(T, \mathscr{G})^{\triangle}$

2) $S_{c}(T, \mathscr{G}) \subset \operatorname{BI}(T, \mathscr{G}, \mathfrak{I} \varphi) u \varphi f=\int f d V\left(\varphi_{+}\right)-\int f d V\left(-\varphi_{-}\right)=\int f d \mathfrak{I} \varphi \partial л$ я каждого $f \in S_{c}(T, \mathscr{G})$ и каждого $\varphi \in S_{c}(T, \mathscr{G})^{\triangle}$.

ДокАЗАТЕЛЬСТво. По следствию 1 из предложения 4 п. $4 \S 2 A \equiv S_{c}(T, \mathscr{G})^{\triangle}$ является решеточным векторным пространством. По теореме 1 из п. $2 \S 2$ $B \equiv \mathfrak{R} \mathfrak{B}(T, \mathscr{G})$ является решеточным векторным пространством.

Если $\varphi, \psi \in A_{+}$и $x, y \in \mathbb{R}_{+}$, то в силу теоремы 1 и теоремы 2 из п. $2 \S 2$ мы имеем

$$
(E \circ V)(x \varphi+y \psi)=E(x V \varphi+y V \psi)=x(E \circ V) \varphi+y(E \circ V) \psi
$$

Кроме того, $E \circ V$ является биективным. Поэтому отображение $E \circ V$ имеет единственное расширение до инъективного линейного оператора $\mathfrak{I}: A \rightarrow B$ такого, что $\mathfrak{I} \varphi=E\left(V\left(\varphi_{+}\right)\right)-E\left(V\left(-\varphi_{-}\right)\right)$для каждого $\varphi \in A$. 
Теперь по предложению 3 из п. $3 \S 2$

$$
\begin{aligned}
S_{c}(T, \mathscr{G}) & \subset \mathrm{BM}_{b c}(T, \mathscr{G}) \subset \mathrm{BI}(T, \mathscr{G}, \Im \varphi) \\
& \cap \mathrm{BI}\left(T, \mathscr{G}, E\left(V\left(\varphi_{+}\right)\right)\right) \cap \mathrm{BI}\left(T, \mathscr{G}, E\left(V\left(-\varphi_{-}\right)\right)\right) .
\end{aligned}
$$

Поэтому по предложениям 1 и 2 из п. $3 \S 2$

$$
\int f d \Im \varphi=\int f d E\left(V\left(\varphi_{+}\right)\right)-\int f d E\left(V\left(-\varphi_{-}\right)\right)=\int f d V\left(\varphi_{+}\right)-\int f d V\left(-\varphi_{-}\right)
$$

для каждой функции $f \in S_{c}(T, \mathscr{G})$. В силу предложения 2 этого пункта $\int f d V\left(\varphi_{+}\right)=\varphi_{+} f$ и $\int f d V\left(-\varphi_{-}\right)=\left(-\varphi_{-}\right) f$. В результате $\int f d \mathfrak{I} \varphi=\varphi_{+} f+$ $\varphi_{-} f=\varphi f$.

По теореме $1 \mathrm{~V}$ сохраняет любые порядковые границы. По теореме 2 из п. $2 \S 2 E$ тоже сохраняет любые порядковые границы. Поэтому $\varphi_{1} \wedge \varphi_{2}=0$ влечет

$$
\Im \varphi_{1} \wedge \mathfrak{I} \varphi_{2}=E\left(V \varphi_{1}\right) \wedge E\left(V \varphi_{2}\right)=E\left(V \varphi_{1} \wedge V \varphi_{2}\right)=E\left(V\left(\varphi_{1} \wedge \varphi_{2}\right)\right)=0 .
$$

По свойству $14 E(b)$ из [12] I является решеточным линейным оператором.

Пусть $\mathfrak{m}$ - любая радоновская бимера. По теореме 2 из п. $2 \S 2 \mathfrak{m}=E \mu^{\prime}-E \mu^{\prime \prime}$ для некоторых мер $\mu^{\prime}, \mu^{\prime \prime} \in \mathfrak{R M}^{w o}(T, \mathscr{G})_{0}$. Согласно теореме 1 этого пункта $\mu^{\prime}=V \varphi^{\prime}$ и $\mu^{\prime \prime}=V \varphi^{\prime \prime}$ для некоторых функционалов $\varphi^{\prime}, \varphi^{\prime \prime} \in A_{+}$. Рассмотрим функционал $\varphi \equiv \varphi^{\prime}-\varphi^{\prime \prime} \in A$. Тогда $\mathfrak{m}=E V \varphi^{\prime}-E V \varphi^{\prime \prime}=\mathfrak{I} \varphi^{\prime}-\mathfrak{I} \varphi^{\prime \prime}=\mathfrak{I} \varphi$. Это означает, что I является сюръективным. Таким образом, I является биективным решеточным линейным оператором. Поэтому $\mathfrak{I}$ - необходимый изоморфизм.

Теперь мы рассмотрим случай равномерно ограниченных функционалов из $S_{c}(T, \mathscr{G})^{\otimes}$.

ТеОрема 3. Пусть $(T, \mathscr{G})$-хаусдорфово пространство. Тогда:

1) отображение $V_{b}$ имеет единственное расширение до изоморфизма $I_{b}$ : $S_{c}(T, \mathscr{G})^{\otimes} \rightarrow \mathfrak{R M}_{b}^{w o}(T, \mathscr{G})$ между данными решеточными векторны.ми пространствами такого, ито $I_{b} \varphi=V_{b}\left(\varphi_{+}\right)-V_{b}\left(-\varphi_{-}\right)$для каждого $\varphi \in S_{c}(T, \mathscr{G}) \otimes_{\text {; }}$

2) $S_{c}(T, \mathscr{G}) \subset \operatorname{BI}\left(T, \mathscr{G}, I_{b} \varphi\right) \quad u \quad \varphi f=\int f d V_{b}\left(\varphi_{+}\right)-\int f d V_{b}\left(-\varphi_{-}\right)=\int f d I_{b} \varphi$ для каждой функиии $f \in S_{c}(T, \mathscr{G})$ и каждого функиионала $\varphi \in S_{c}(T, \mathscr{G}) \otimes$;

3) отображение $E_{b} \circ V_{b}$ имеет единственное расширение до изоморфизма $\mathfrak{I}_{b}: S_{c}(T, \mathscr{G})^{\ominus} \rightarrow \mathfrak{R B}_{b}(T, \mathscr{G})$ между данными решеточными векторными пространствами такого, что $\mathfrak{I}_{b}=\mathfrak{I} \mid S_{c}(T, \mathscr{G}) \otimes \quad$ и $\mathfrak{I}_{b} \varphi=E_{b} V_{b}\left(\varphi_{+}\right)-$ $E_{b} V_{b}\left(-\varphi_{-}\right)=F_{b} I_{b} \varphi ;$

4) $S_{c}(T, \mathscr{G}) \subset \mathrm{BI}\left(T, \mathscr{G}, \mathfrak{I}_{b} \varphi\right)$ u $\varphi f=\int f d \mathfrak{I}_{b} \varphi$ для каждой функции $f \in S_{c}(T, \mathscr{G})$ и каждого функционала $\varphi \in S_{c}(T, \mathscr{G}) \otimes$.

ДокАЗАТЕльство. По следствию 3 предложения 4 из п. $4 \S 2 A \equiv S_{c}(T, \mathscr{G}) \otimes$ является решеточным векторным пространством. По предложению из п. $5 \S 1$ $B \equiv \mathfrak{R M}_{b}^{w o}(T, \mathscr{G})$ также является решеточным векторным пространством. Поэтому в силу теоремы 1 этого пункта и предложения 3.6.1 из [24] отображение $V_{b}$ имеет единственное расширение до инъективного линейного оператора $I_{b}: A \rightarrow B$ такого, что $I_{b} \varphi=V_{b}\left(\varphi_{+}\right)-V_{b}\left(-\varphi_{-}\right)$. 
По теореме $1 V_{b}$ сохраняет любые порядковые границы. Поэтому $\varphi_{1} \wedge \varphi_{2}=0$ влечет $I_{b} \varphi_{1} \wedge I_{b} \varphi_{2}=V_{b} \varphi_{1} \wedge V_{b} \varphi_{2}=V_{b}\left(\varphi_{1} \wedge \varphi_{2}\right)=0$. По свойству $14 E(b)$ из [12] $I_{b}$ является решеточным линейным оператором.

Если $\mu \in B$, то $\mu=\mu_{+}-\left(-\mu_{-}\right)$. Согласно теореме $1 \mu_{+}=V_{b} \varphi^{\prime}$ и $-\mu_{-}=V_{b} \varphi^{\prime \prime}$ для некоторых $\varphi^{\prime}, \varphi^{\prime \prime} \in A_{+}$. Рассмотрим функционал $\varphi \equiv \varphi^{\prime}-\varphi^{\prime \prime} \in A$. Тогда $\mu=V_{b} \varphi^{\prime}-V_{b} \varphi^{\prime \prime}=I_{b} \varphi^{\prime}-I_{b} \varphi^{\prime \prime}=I_{b} \varphi$. Это означает, что $I_{b}$ является сюръективным. Таким образом, $I_{b}$ есть биективный решеточный линейный оператор. Поэтому $I_{b}$ есть необходимый изоморфизм. В результате мы получаем утверждение 1).

Если $f \in S_{c}(T, \mathscr{G})$ и $\varphi \in A$, то по предложению 2 этого пункта и следствию из него функция $f$ интегрируема относительно мер $\mu^{\prime} \equiv V_{b}\left(\varphi_{+}\right)$и $\mu^{\prime \prime} \equiv V_{b}\left(-\varphi_{-}\right)$ из $B$ и, кроме того, $\left(\varphi_{+}\right) f=\int f d \mu^{\prime}$ и $\left(-\varphi_{-}\right) f=\int f d \mu^{\prime \prime}$. Поэтому $f \in \operatorname{BI}(T, \mathscr{G}$, $\left.\mu^{\prime}-\mu^{\prime \prime}\right)=\operatorname{BI}\left(T, \mathscr{G}, I_{b} \varphi\right)$ и $\varphi f=\left(\varphi_{+}\right) f-\left(-\varphi_{-}\right) f=\int f d \mu^{\prime}-\int f d \mu^{\prime \prime}=$ $\int f d\left(\mu^{\prime}-\mu^{\prime \prime}\right)=\int f d I_{b} \varphi$. Таким образом, мы получаем утверждение 2$)$.

По теореме 1 из п. $2 \S 2 \quad C \equiv \mathfrak{R B}_{b}(T, \mathscr{G})$ является решеточным векторным пространством. Если $\varphi, \psi \in A_{+}$и $x, y \in \mathbb{R}_{+}$, то в силу теоремы 1 и теоремы 2 из п. $2 \S 2$ мы имеем

$$
\left(E_{b} \circ V_{b}\right)(x \varphi+y \psi)=E_{b}\left(x V_{b} \varphi+y V_{b} \psi\right)=x\left(E_{b} \circ V_{b}\right) \varphi+y\left(E_{b} \circ V_{b}\right) \psi .
$$

Кроме того, $E_{b} \circ V_{b}$ биективно. Поэтому отображение $E_{b} \circ V_{b}$ имеет единственное расширение до инъективного линейного оператора $\mathfrak{I}_{b}: A \longmapsto C$ такого, что $\mathfrak{I}_{b} \varphi=E_{b} V_{b}\left(\varphi_{+}\right)-E_{b} V_{b}\left(-\varphi_{-}\right)$. Используя теорему 3 из п. $2 \S 2$, мы получаем $\Im_{b} \varphi=F_{b}\left(V_{b}\left(\varphi_{+}\right)-V_{b}\left(-\varphi_{-}\right)\right)=F_{b} I_{b} \varphi$ для каждого $\varphi \in A$.

По утверждению 1) $I_{b}: A \nrightarrow B$ есть изоморфизм. По теореме 3 из п. $2 \S 2$ $F_{b}: B \longmapsto C$ есть также изоморфизм. Поэтому из $\mathfrak{I}_{b}=F_{b} \circ I_{b}$ мы заключаем, что $\mathfrak{I}_{b}: A \longmapsto C$ является изоморфизмом данных решеточных векторных пространств.

Согласно п. $2 E_{b}=E \mid B$. Согласно п. $4 \S 1 V_{b}=V \mid A_{+}$. Поэтому для каждого $\varphi \in A$ мы имеем

$$
\begin{aligned}
\mathfrak{I} \varphi & =E V\left(\varphi_{+}\right)-E V\left(-\varphi_{-}\right)=E V_{b}\left(\varphi_{+}\right)-E V_{b}\left(-\varphi_{-}\right) \\
& =E_{b} V_{b}\left(\varphi_{+}\right)-E_{b} V_{b}\left(-\varphi_{-}\right)=\mathfrak{I}_{b} \varphi
\end{aligned}
$$

откуда $\mathfrak{I}_{b}=\mathfrak{I} \mid A$. Таким образом, мы получаем утверждение 3$)$.

Наконец, из равенства $\mathfrak{I}_{b}=\mathfrak{I} \mid A$ мы выводим по теореме 2 этого пункта, что $S_{c}(T, \mathscr{G}) \subset \mathrm{BI}\left(T, \mathscr{G}, \Im_{b} \varphi\right)$ для каждого $\varphi \in A$ и $\varphi f=\int f d \Im_{b} \varphi$ для каждого $f \in S_{c}(T, \mathscr{G})$.

В добавление к теореме 3 мы докажем, что отображения $I_{b} \quad$ и $\mathfrak{I}_{b}$ сохраняют соответствуюшие нормы.

Так как $S_{c}(T, \mathscr{G})^{\ominus} \subset\left(S_{c}(T, \mathscr{G}),\|\cdot\|_{u}\right)^{\prime}$, то мы можем индуцировать на решеточное векторное пространство $S_{c}(T, \mathscr{G}) \otimes$ норму $\|\cdot\|$ из нормированного решеточного векторного пространства $\left(\left(S_{c}(T, \mathscr{G}),\|\cdot\|_{u}\right)^{\prime},\|\cdot\|\right)$.

ПРЕДЛОЖЕНИЕ 3. Пусть $(T, \mathscr{G})$ - Хаусдорфово пространство. Тогда:

1) $\left(S_{c}(T, \mathscr{G})^{\ominus},\|\cdot\|\right)$ является нормированным решеточным векторнылм пространством; 
2) отображсение $I_{b}:\left(S_{c}(T, \mathscr{G})^{\ominus},\|\cdot\|\right) \longmapsto(\mathfrak{R M}$ wo $(T, \mathscr{G}),\|\cdot\|)$ является (изометрическим) изоморфизмом межсду данными нормированными решеточными векторными пространствами;

3) отображсние $\mathfrak{I}_{b}:\left(S_{c}(T, \mathscr{G})^{\ominus},\|\cdot\|\right) \longmapsto\left(\mathfrak{R B}_{b}(T, \mathscr{G}),\|\cdot\|\right)$ является (изометрическим) изоморфизмом межсду данными нормированнымм решеточными векторным.м пространствами.

ДоказАТЕльство. 1) Обозначим $S_{c}(T, \mathscr{G})$ через $A(T), A(T)^{\ominus}$ через $X$ и $\left(A(T),\|\cdot\|_{u}\right)^{\prime}$ через $Y$.

Нам необходимо проверить, что $\|\cdot\|$ является монотонной нормой на $X$. Известно, что $(Y,\|\cdot\|)$ является банаховым решеточным векторным пространством. По следствию 1 из предложения 4 п. $4 \S 2$ наименьшие верхние (наибольшие нижние) границы совпадают в $X$ и $A(T)^{\sim}$. Аналогично они совпадают в $Y$ и $A(T)^{\sim}$. Поэтому эти гранищы совпадают в $X$ и $Y$. Следовательно, $|\varphi|_{X}=|\varphi|_{Y}$ для каждого $\varphi \in X$. В результате $|\varphi|_{X} \leqslant|\psi|_{X}$ в $X$ влечет $\|\varphi\| \leqslant\|\psi\|$, поскольку норма $\|\cdot\|$ является монотонной в $Y$. Значит, $(X,\|\cdot\|)$ является нормированным решеточным векторным пространством.

2) Пусть $\varphi \in X_{+}, \mu_{0}=V_{b} \varphi$ и $f \in A(T)$. Тогда $|\varphi f| \leqslant \int|f| d \mu_{0} \leqslant\|f\|_{u} \int \mathbf{1} d \mu=$ $\|f\|_{u} \mu_{0} T=\|f\|_{u}\left\|\mu_{0}\right\|$. Следовательно, $\|\varphi\| \leqslant\left\|\mu_{0}\right\|$.

Согласно доказательству предложения $2 \mu_{0} T=\sup \left\{\lambda K \mid K \in \mathscr{I}_{\sigma}(A(T))\right\}$ и $\lambda K=\inf \left\{\varphi f \mid f \in A(T)_{+} \wedge f \geqslant \chi(K)\right\}$. Так как $A(T)$ является усекаемым семейством и функционал $\varphi$ возрастает, мы получаем $\lambda K=\inf \left\{\varphi f \mid f \in A(T)_{+} \wedge\right.$ $(\chi(K) \leqslant f \leqslant \mathbf{1})\}$, откуда $\varphi f=|\varphi f| \leqslant\|\varphi\|\|f\|_{u} \leqslant\|\varphi\|$ влечет $\lambda K \leqslant\|\varphi\|$. В результате $\left\|\mu_{0}\right\|=\mu_{0} T \leqslant\|\varphi\|$. Таким образом, $\|\varphi\|=\left\|\mu_{0}\right\|$.

Пусть теперь $\varphi \in X$ и $\mu \equiv I_{b} \varphi$. Тогда $|\mu|=I_{b}|\varphi|=V_{b}|\varphi| . \quad$ В силу равенства, доказанного выше, мы получаем $\|\varphi\|=\||\varphi|\|=\||\mu|\|=\|\mu\|$. Таким образом, $I_{b}$ является изометрическим. Значит, $I_{b}$ есть необходимьй изоморфизм нормированных решеточных векторных пространств.

3) По следствию из предложения 6 п. $2 \oint 2 \quad F_{b}:\left(\Re_{\mathfrak{m}}^{w o}(T, \mathscr{G}),\|\cdot\|\right) \longmapsto$ $(\mathfrak{R B}(T, \mathscr{G}),\|\cdot\|)$ является (изометрическим) изоморфизмом данных нормированных решеточных векторных пространств. Кроме того, по теореме 3 этого пункта $\mathfrak{I}_{b}=F_{b} \circ I_{b}$. Следовательно, $\mathfrak{I}_{b}$ также является необходимым (изометрическим) изоморфизмом.

Теоремы 1-3 и предложение 3 дают решение проблемы обшего радоновского представления. В заключение мы дадим изоморфную версию первой теоремы Рисса-Радона о представлении.

TЕОРема 4. Пусть $(T, \mathscr{G})$ - локально компактное пространство $и W$ : $\left(C_{c}(T, \mathscr{G})^{\sim}\right)_{+} \longmapsto \mathfrak{R M}^{\text {wo }}(T, \mathscr{G})_{0}$ - биекиия из теоремы Рисса-Радона о представлении, приведенной во введении. Тогда:

1) отображение $E \circ W$ имеет единственное расширение до изоморфизма $\mathfrak{J}: C_{c}(T, \mathscr{G})^{\sim} \rightarrow \mathfrak{R B}(T, \mathscr{G})$ между даннылми решеточным.ми векторными пространствами такого, что $\mathfrak{J} \varphi=E\left(W\left(\varphi_{+}\right)\right)-E\left(W\left(-\varphi_{-}\right)\right)$для каждого функиионала $\varphi \in C_{c}(T, \mathscr{G})^{\sim}$;

2) $C_{c}(T, \mathscr{G}) \subset \operatorname{BI}(T, \mathscr{G}, \mathfrak{J} \varphi) u \varphi f=\int f d W\left(\varphi_{+}\right)-\int f d W\left(-\varphi_{-}\right)=\int f d \mathfrak{J} \varphi \partial \Omega$ я каждой функиии $f \in C_{c}(T, \mathscr{G})$ и каждого функиионала $\varphi \in C_{c}(T, \mathscr{G})^{\sim}$. 
ДокаЗАТЕЛЬСТво. Напомним, что $A \equiv C_{c}(T, \mathscr{G})^{\sim}$ и $B \equiv \mathfrak{R} \mathfrak{B}(T, \mathscr{G})$ являются решеточными векторными пространствами.

Пусть $\mu \equiv W \varphi, \quad \nu \equiv W \psi \quad$ и $\quad x, y \in \mathbb{R}_{+} . \quad$ По предложению из п. $5 \S 1$ $\lambda \equiv x \mu+y \nu \in \mathfrak{R M}^{w o}(T, \mathscr{G})_{0}$. По теореме Рисса-Радона $C_{c}(T, \mathscr{G}) \subset \mathrm{MI}\left(T, \mathscr{M}_{0}, \mu\right) \cap$ $\operatorname{MI}\left(T, \mathscr{M}_{0}, \nu\right)$, где $\mathscr{M}_{0} \equiv \mathscr{B}(T, \mathscr{G})$. Известно, что $\mathrm{MI}\left(T, \mathscr{M}_{0}, \lambda\right)=\operatorname{MI}\left(T, \mathscr{M}_{0}, \mu\right) \cap$ $\operatorname{MI}\left(T, \mathscr{M}_{0}, \nu\right) \quad$ и $\quad \Lambda(\lambda) f=x \Lambda(\mu) f+y \Lambda(\nu) f$ для каждого $f \in \operatorname{MI}\left(T, \mathscr{M}_{0}, \lambda\right)$. Поэтому $C_{c}(T, \mathscr{G}) \subset \operatorname{MI}\left(T, \mathscr{M}_{0}, \lambda\right)$, и для каждого $f \in C_{c}(T, \mathscr{G})$ мы имеем $(x \varphi+y \psi) f=x \int f d \mu+y \int f d \nu=\int f d \lambda$. Поэтому $\lambda=W(x \varphi+y \psi)$. В результате $E W(x \varphi+y \psi)=x E W \varphi+y E W \psi$ по теореме 2 из п. $2 \S 2$.

Кроме того, $E \circ W$ биективно. Поэтому в силу предложения 3.6 .1 из [24] отображение $E \circ W$ имеет единственное расширение до инъективного линейного оператора $\mathfrak{J}: A \rightarrow B$ такого, что $\mathfrak{J} \varphi=E\left(W\left(\varphi_{+}\right)\right)-E\left(W\left(-\varphi_{-}\right)\right)$для каждого $\varphi \in A$.

Теперь по предложению 3 из п. $3 \S 2$

$$
\begin{aligned}
C_{c}(T, \mathscr{G}) & \subset \mathrm{BM}_{b c}(T, \mathscr{G}) \subset \mathrm{BI}(T, \mathscr{G}, \mathfrak{J} \varphi) \\
& \cap \mathrm{BI}\left(T, \mathscr{G}, E\left(W\left(\varphi_{+}\right)\right)\right) \cap \mathrm{BI}\left(T, \mathscr{G}, E\left(W\left(-\varphi_{-}\right)\right)\right) .
\end{aligned}
$$

Поэтому по предложениям 1 и 2 из п. $3 \S 2$

$$
\int f d \mathfrak{J} \varphi=\int f d E\left(W\left(\varphi_{+}\right)\right)-\int f d E\left(W\left(-\varphi_{-}\right)\right)=\int f d W\left(\varphi_{+}\right)-\int f d W\left(-\varphi_{-}\right)
$$

для каждого $f \in C_{c}(T, \mathscr{G})$. Так как $\int f d W\left(\varphi_{+}\right)=\varphi_{+} f$ и $\int f d W\left(-\varphi_{-}\right)=\left(-\varphi_{-}\right) f$, то в результате $\int f d \mathfrak{J} \varphi=\varphi_{+} f+\varphi_{-} f=\varphi f$.

Пусть $\varphi, \psi \in A_{+}$. Если $\varphi \leqslant \psi$, то $\psi-\varphi \geqslant 0$. Поэтому $E W \psi-E W \varphi=\mathfrak{J} \psi-$ $\mathfrak{J} \varphi=\mathfrak{J}(\psi-\varphi)=E W(\psi-\varphi) \geqslant 0$ влечет $E W \varphi \leqslant E W \psi$. Значит, отображение $E \circ W$ является возрастающим. Обратно: пусть $E W \varphi \leqslant E W \psi$. По теореме 2 из п. $2 \S 2$ отображение $E$ изотонно. Поэтому $W \varphi \leqslant W \psi$. Следовательно, $\varphi f=\int f d W \varphi \leqslant \int f d W \psi=\psi f$ для каждого $f \in C_{c}(T, \mathscr{G})$. Это означает, что $\varphi \leqslant \psi$. Таким образом, отображение $E \circ W$ изотонно. Поэтому оно сохраняет любые порядковые границы.

Теперь $\varphi_{1} \wedge \varphi_{2}=0$ в $A_{+}$влечет

$$
\mathfrak{J} \varphi_{1} \wedge \mathfrak{J} \varphi_{2}=(E \circ W) \varphi_{1} \wedge(E \circ W) \varphi_{2}=(E \circ W)\left(\varphi_{1} \wedge \varphi_{2}\right)=0
$$

По свойству $14 E(b)$ из [12] J является решеточным линейным оператором.

Пусть $\mathfrak{m}$ - любая радоновская бимера. По теореме 2 из п. $2 \S 2 \mathfrak{m}=E \mu^{\prime}-E \mu^{\prime \prime}$ для некоторых мер $\mu^{\prime}, \mu^{\prime \prime} \in \mathfrak{R M}^{w o}(T, \mathscr{G})_{0}$. По теореме Рисса-Радона $\mu^{\prime}=W \varphi^{\prime}$ и $\mu^{\prime \prime}=W \varphi^{\prime \prime}$ для некоторых функционалов $\varphi^{\prime}, \varphi^{\prime \prime} \in A_{+}$. Рассмотрим функционал $\varphi \equiv \varphi^{\prime}-\varphi^{\prime \prime} \in A$. Тогда $\mathfrak{m}=E W \varphi^{\prime}-E W \varphi^{\prime \prime}=\mathfrak{J} \varphi^{\prime}-\mathfrak{J} \varphi^{\prime \prime}=\mathfrak{J} \varphi$. Это означает, что $\mathfrak{J}$ сюръективно. Таким образом, $\mathfrak{J}$ является биективным решеточным линейным оператором. Поэтому $\mathfrak{J}$ есть необходимый изоморфизм. 
ЗАмечание 1. Результаты, аналогичные предложениям 1 и 2 этого пункта, могут быть доказаны для любого топологически обширного $\mathscr{F}$-усекаемого решеточного векторного пространства $A(T)$ на хаусдорфовом пространстве $(T, \mathscr{G})$, в


дение, параллельное теореме Прохорова о представлении из п. $7 \S$ 1: интегральное представление устанавливает некоторые изоморфизмы $(S(T, \mathscr{G}) \otimes,\|\cdot\|) \mapsto$ $\left(\mathfrak{R M}_{b}^{w o}(T, \mathscr{G}),\|\cdot\|\right)$ u $\left(S(T, \mathscr{G})^{\otimes},\|\cdot\|\right) \nrightarrow\left(\mathfrak{R B}_{b}(T, \mathscr{G}),\|\cdot\|\right)$ между данными нормированными решеточными векторными пространствами, которые являются аналогами изоморфизмов из теоремы 3 и предложсения 3 әтого пункта.

ЗАмЕчАнИЕ 2. Если мы в определении положительной радоновской меры $\mu$ : $\mathscr{M} \rightarrow \overline{\mathbb{R}}_{+}$заменим условие локальной ограниченности на более слабое условие: $\mu C<\infty$ для каждого $C \in \mathscr{C}$ (конечность меры на компактах), то такую меру назовем обобщенной положительной радоновской мерой. Для локально компактного пространства понятия положительной радоновской меры и обобшенной положительной радоновской меры равносильны. Понятие обобщенной положительной радоновской меры естественным образом влечет понятие обобщенной радоновской бимеры.

Далее, если мы в определении тонкого функционала отбросим условие локальной ограниченности, то такой функционал назовем обобщенным тонким функционалом.

Соответственно этим обобшениям все изложенные теоремы остаются в силе для обобщенных радоновских мер и бимер и обобщенных тонких функционалов.

\section{Список литературы}

1. Riesz F. Sur les opérations fonctionelles linéaires // C. R. Acad. Sci. Paris. 1909. V. 149. P. 974-977.

2. Radon J. Theorie und Anwendangen der absolut additiven Mengenfunktionen // S.-B. Akad. Wiss. Wien. 1913. V. 122. P. 1295-1438.

3. Hausdorff F. Grundzuge der Mengenlehre. Leipzig: Veit, 1914.

4. Saks $S$. Theory of the integral. Warszawa, 1937.

5. Saks $S$. Integration in abstract metric spaces // Duke Math. J. 1938. V. 4. P. 408-411.

6. Kakutani S. Concrete representation of abstract (M) - spaces // Ann. Math. (2) 1941. V. 42. P. 994-1024.

7. Halmos P. R. Measure Theory. New York, 1950.

8. Hewitt E. Integration on locally compact spaces. I // Univ. of Washington Publ. in Math. 1952. V. 3. P. $71-75$.

9. Edwads R. E. A theory of Radon measures on locally compact spaces // Acta Math. 1953. V. 89. P. $133-164$.

10. Bourbaki N. Intégration. Chap. I-VI. Paris: Hermann, 1952-1959.

11. Hewitt E., Stromberg K. Real and Abstract Analysis. Berlin: Springer-Verlag, 1965.

12. Fremlin D. H. Topological Riesz spaces and measure theory. Cambridge: The University Press, 1974.

13. Прохоров Ю.В. Сходимость случайных процессов и предельные теоремы теории вероятностей // Теория вероятностей и ее применения. 1956. Т. 1. С. 177-238.

14. Topsoe F. Topology and measure // Lect. Notes Math. 1970. V. 133.

15. Pollard D., Topsoe F. A unified approach to Riesz type representation theorems // Studia Math. 1975. V. 54. P. 173-190.

16. Topsoe F. Further results on integral representations // Studia Math. 1976. V. 55. P. 239-245. 
17. Topsoe F. Radon measures, some basic constructions // Lect. Notes Math. 1983. V. 1033. P. 303-311.

18. Anger B., Portenier C. Radon integrals // Progress in Math. № 103. Boston-Berlin: Birkhäuser 1992.

19. König H. The Danielle-Stone-Riesz representation theorem // Operator theory: advances and applications. 1995. V. 75. P. 191-222.

20. König H. Measure and integration. Berlin: Springer-Verlag, 1997.

21. Захаров B. K., Михалёв А. В. Проблема Радона для регулярных мер на произвольном хаусдорфовом пространстве // Фундаментальная и прикладная математика. 1997. Т. 3. №3. C. 801-808.

22. Захаров В.К., Михалёв А.В. Интегральное представление для радоновских мер на произвольном хаусдорфовом пространстве // Фундаментальная и прикладная математика. 1997. Т. 3. № 4. С. 1135-1172.

23. Захаров В. К., Михалёв А. В. Проблема интегрального представления для радоновских мер на произвольном хаусдорфовом пространстве // Докл. РАН. 1998. Т. 360. № 1. C. $13-15$.

24. Semadeni Z. Banach spaces of continuous functions. Warszawa: Polish Sci. Publ., 1971.

25. Dinculianu N. Vector Measures. London-N.Y.: Oxford Univ. Press (Pergamon), 1967.

26. Александров А. Д. Additive functions in abstract spaces. I-III // Матем. сб. 1940. Т. 8. C. $303-348 ; 1941$. T. 9.

27. Stone M. N. Notes on integration. I-IV // Proc. Nat. Acad. Sci. USA. 1948. V. 34. P. 336-342, 447-455, 483-490; 1949. V. 35. P. 50-58.

28. Schwartz L. Radon measures on arbitrary topological spaces and cylindrical measures. London-N.Y.: Oxford Univ. Press, 1973.

29. Jacobs K. Measure and integral. N.Y.: Academic Press, 1978.

30. Канторович Л. В., Акилов Г. П. Функциональный анализ. М.: Наука, 1977.

31. Захаров В. К. Функциональное представление равномерного пополнения максимального и счетно-плотного модулей частных модуля непрерывных функций // УМН. 1980. Т. 35. № 4. C. $187-188$.

32. Zaharov V. Functional characterization of absolute and Dedekind completion // Bull. Acad. Polon. Sci. Serie sci. math. 1981. V. 29. № 5-6. P. 293-297.

33. Захаров B. K. Связи между расширением Лебега и расширением Бореля первого класса и между соответствующими им прообразами // Изв. АН СССР. Сер. матем. 1990. T. 54. № 5. C. 928-956.

34. Hausdorff F. Über halbstetige Functionen und deren Verallgemeinerung // Math. Z. 1915. V. 4. P. 292-309.

35. Sierpin'ski $W$. Sur les fonctions développables en séries absolument convergentes de fonctions continues // Fund. Math. 1921. V. 2. P. 15-27.

36. Zaharov V.K. Alexandrovian cover and Sierpin'skian extension // Studia Sci. Math. Hung. 1989. V. 24. P. 93-117.

37. Захаров В.К. Связь между полным кольцом частных кольца непрерывных функций, регулярным пополнением и расширениями Хаусдорфа-Серпинского // УМН. 1990. T. 45. №6. C. 133-134.

38. Захаров В.К. Расширения кольца непрерывных функций, порожденные регулярным, счетно-делимым и полным кольцами частных, и соответствующие им прообразы // Изв. РАН. Сер. матем. 1995. Т. 59. № 4. С. 15-60.

Центр новых информационных технологий

Поступило в редакцию

Московского государственного университета

19.XII.1997

им. М. В. Ломоносова 